\title{
Worst-case response time analysis of real-time tasks under fixed-priority scheduling with deferred preemption
}

\author{
Reinder J. Bril • Johan J. Lukkien • \\ Wim F.J. Verhaegh
}

Published online: 28 April 2009

(C) The Author(s) 2009. This article is published with open access at Springerlink.com

\begin{abstract}
Fixed-priority scheduling with deferred preemption (FPDS) has been proposed in the literature as a viable alternative to fixed-priority pre-emptive scheduling (FPPS), that obviates the need for non-trivial resource access protocols and reduces the cost of arbitrary preemptions.

This paper shows that existing worst-case response time analysis of hard real-time tasks under FPDS, arbitrary phasing and relative deadlines at most equal to periods is pessimistic and/or optimistic. The same problem also arises for fixed-priority nonpre-emptive scheduling (FPNS), being a special case of FPDS. This paper provides a revised analysis, resolving the problems with the existing approaches. The analysis is based on known concepts of critical instant and busy period for FPPS. To accommodate for our scheduling model for FPDS, we need to slightly modify existing definitions of these concepts. The analysis assumes a continuous scheduling model, which is based on a partitioning of the timeline in a set of non-empty, right semi-open intervals. It is shown that the critical instant, longest busy period, and worst-case response time for a task are suprema rather than maxima for all tasks, except for the lowest priority task. Hence, that instant, period, and response time cannot be assumed for any task, except for the lowest priority task. Moreover, it is shown that the analysis is not uniform for all tasks, i.e. the analysis for the lowest priority task differs from the
\end{abstract}

Excerpts of this document have been published as Bril et al. (2007).

R.J. Bril $(\bowtie) \cdot$ J.J. Lukkien

Department of Mathematics and Computer Science, Technische Universiteit Eindhoven (TU/e),

Den Dolech 2, 5600 AZ, Eindhoven, The Netherlands

e-mail: r.j.bril@tue.nl

J.J. Lukkien

e-mail: j.j.lukkien@tue.nl

W.F.J. Verhaegh

Philips Research Laboratories, High Tech Campus 11, 5656 AE, Eindhoven, The Netherlands

e-mail: wim.verhaegh@philips.com 
analysis of the other tasks. These anomalies for the lowest priority task are an immediate consequence of the fact that only the lowest priority task cannot be blocked. To build on earlier work, the worst-case response time analysis for FPDS is expressed in terms of known worst-case analysis results for FPPS. The paper includes pessimistic variants of the analysis, which are uniform for all tasks, illustrates the revised analysis for an advanced model for FPDS, where tasks are structured as flow graphs of subjobs rather than sequences, and shows that our analysis is sustainable.

Keywords Level- $i$ active period - Level- $i$ busy period - Worst-case response time Worst-case occupied time $\cdot$ Periodic tasks $\cdot$ Fixed-priority scheduling $\cdot$ Deferred preemption $\cdot$ Real-time systems

\section{Introduction}

\subsection{Motivation}

Based on the seminal paper of Liu and Layland (1973), many results have been achieved in the area of analysis for fixed-priority preemptive scheduling (FPPS). Arbitrary preemption of real-time tasks has a number of drawbacks, though. In systems requiring mutual access to shared resources, arbitrary preemptions induce the need for non-trivial resource access protocols, such as the priority ceiling protocol (Sha et al. 1990). In systems using cache memory, e.g. to bridge the speed gap between processors and main memory, arbitrary preemptions induce additional cache flushes and reloads. As a consequence, system performance and predictability are degraded, complicating system design, analysis and testing (Burns and Wellings 1997; Gopalakrishnan and Parulkar 1996; Lee et al. 1998; Mok and Poon 2005; Simonson and Patel 1995). Although fixed-priority non-preemptive scheduling (FPNS) may resolve these problems, it generally leads to reduced schedulability compared to FPPS. Therefore, alternative scheduling schemes have been proposed between the extremes of arbitrary preemption and no preemption. These schemes are also known as deferred preemption or co-operative scheduling (Burns 1994), and are denoted by fixedpriority scheduling with deferred preemption (FPDS) in the remainder of this paper.

Worst-case response time analysis of periodic real-time tasks under FPDS, arbitrary phasing, and relative deadlines within periods has been addressed in a number of papers (Bril et al. 2004; Burns 1994; Burns and Wellings 1997; Lee et al. 1998). The existing analysis is not exact, however. In Bril et al. (2004), it has already been shown that the analysis presented in Burns (1994), Burns and Wellings (1997), Lee et al. (1998) is pessimistic. More recently, it has been shown in Bril (2006) that the analysis presented in Bril et al. (2004), Burns (1994), Burns and Wellings (1997) is optimistic. Unlike the implicit assumptions in those latter papers, the worst-case response time of a task under FPDS and arbitrary phasing is not necessarily assumed for the first job of that task upon its critical instant. Hence, the existing analysis may provide guarantees for tasks that in fact miss their deadlines in the worst-case. In Bril et al. (2006), it has been shown that the latter problem also arises for FPNS, being a special case of FPDS, and its application for the schedulability analysis of controller area networks (CAN) (Tindell and Burns 1994; Tindell et al. 1994, 1995). Revised 
analysis for CAN resolving the problem with the original approach in an evolutionary fashion can be found in Davis et al. (2007).

\subsection{Contributions}

This paper resolves the problems with the existing approaches by presenting a novel worst-case response time analysis for hard real-time tasks under FPDS, arbitrary phasing and arbitrary relative deadlines. The analysis assumes a continuous scheduling model rather than a discrete scheduling model (Baruah et al. 1990b), e.g. all task parameters are taken from the real numbers. The motivation for this assumption stems from the observation that a discrete view on time is in many situations insufficient; see for example Baeten and Middelburg (2002), Hooman (1991), Koymans (1990). The scheduling model is based on a partitioning of the timeline in a set of non-empty, right semi-open intervals (Buttazzo 2005; Hooman 1991). The analysis is based on the concepts of critical instant (Liu and Layland 1973) and busy period (Lehoczky 1990). To accommodate for our scheduling model for FPDS, we need to slightly modify the existing definitions of these concepts. To prevent confusion with the existing definition of busy period, we use the term active period in this document, for which we give a formal definition.

In this document, we discuss conditions for termination of an active period, and present a sufficient condition with a formal proof. Moreover, we show that the critical instant, longest active period, and worst-case response time for a task are suprema rather than maxima for all tasks, except for the lowest priority task. Hence, that instant, period, and response time cannot be assumed for any task, except for the lowest priority task. Our worst-case response time analysis is not uniform for all tasks. In particular, the analysis for the lowest priority task differs from the analysis for the other tasks. These anomalies for the lowest priority task are an immediate consequence of the fact that, unlike the other tasks, the lowest priority task cannot be blocked. To build on earlier results, worst-case response times under FPDS are expressed in terms of worst-case response times and worst-case occupied times (Bril 2004) under FPPS. We also present pessimistic variants of the analysis, which are indeed uniform for all tasks, and show that the revised analysis for CAN presented in Davis et al. (2007) conforms to a pessimistic variant. We illustrate our analysis for an advanced model for FPDS, where tasks are structured as flow graphs of subjobs rather than sequences, and we show that our analysis for FPDS is sustainable (Baruah and Burns 2006) and therefore also applicable for sporadic task systems (Mok 1983; Baruah et al. 1990a).

\subsection{Related work}

Next to continuous scheduling models, one can find discrete scheduling models in the literature, e.g. in George et al. (1996), Hermant et al. (1996), and models in which domains are not explicitly specified (Buttazzo 2005; Klein et al. 1993; Liu 2000). Because the equations for response time analysis depend on the model, we prefer to be explicit about the domains in our model. As mentioned above, our scheduling model is based on a partitioning of the timeline in a set of non-empty, right semi-open intervals. Alternatively, the scheduling model in Liu (2000) is based on left semi-open intervals. 
In this paper, we assume that each job (or activation) of a task consists of a sequence of non-preemptable subjobs, where each subjob has a known worst-case computation time, and we present a novel worst-case response time analysis to determine schedulability of tasks under FPDS. Similarly, George et al. (1996) assume that the worst-case computation time of each non-preemptive job is known, and present worst-case response time analysis of tasks under FPNS. Conversely, Baruah (2005) determines the largest non-preemptive 'chunks' into which jobs of a task can be broken up to still ensure feasibility under earliest deadline first (EDF).

For worst-case response time analysis of tasks under FPPS, arbitrary phasing, and relative deadlines at most equal to periods, it suffices to determine the response time of the first job of a task upon its critical instant. For tasks with relative deadlines larger than their respective periods, Lehoczky (1990) introduced the concept of a busy period, and showed that all jobs of a task in a busy period need to be considered to determine its worst-case response time. Hence, when the relative deadline of a task is larger than its period, the worst-case response time of that task is not necessarily assumed for the first job of a task when released at a critical instant. Similarly, González Harbour et al. (1991) showed that if relative deadlines are at most equal to periods, but priorities vary during execution, then again multiple jobs must be considered to determine the worst-case response time. Initial work on pre-emption thresholds (Wang and Saksena 1999) failed to identify this issue. The resulting flaw was later corrected by Regehr (2002). Worst-case response time analysis of tasks under EDF and relative deadlines at most equal to periods described by Spuri (1996) is also based on the concept of busy period.

\subsection{Structure}

This paper has the following structure. First, in Sect. 2, we present basic real-time scheduling models for FPPS and FPDS, in which we assume fixed values for computation times and periods. These assumptions ease the presentation in subsequent sections, and will be lifted in Sect. 8. Next, worst-case analysis for FPPS is briefly recapitulated in Sect. 3. Section 4 presents various examples refuting the existing worst-case response time analysis for FPDS. The notion of active period is the topic of Sect. 5. We present a formal definition of active period and theorems with a recursive equation for the length of an active period and an iterative procedure to determine its value. Worst-case analysis for FPDS is addressed in Sect. 6. We present a theorem for critical instant and theorems to determine the worst-case response time of a task under FPDS, arbitrary phasing, and arbitrary relative deadlines. Section 7 illustrates the worst-case response time analysis by applying it to some examples presented in Sect. 4. Section 8 compares the notion of level- $i$ active period with similar definitions in the literature, and presents pessimistic variants of the worst-case response time analysis for FPDS. Moreover, this section illustrates the revised analysis for an advanced model for FPDS and briefly discusses the sustainability of the analysis, i.e. we show that our schedulability analysis remains valid if we replace fixed computation times by worst-case computation times and fixed periods by minimal inter-arrival times (or worst-case periods) of tasks in our real-time scheduling models. The paper is concluded in Sect. 9. 


\section{Real-time scheduling models}

This section starts with a presentation of a basic real-time scheduling model for FPPS. Next, that basic model is refined for FPDS. The section is concluded with remarks.

\subsection{Basic model for FPPS}

We assume a single processor and a set $\mathcal{T}$ of $n$ periodically released, independent tasks $\tau_{1}, \tau_{2}, \ldots, \tau_{n}$ with unique, fixed priorities. At any moment in time, the processor is used to execute the highest priority task that has work pending. So, when a task $\tau_{i}$ is being executed, and a release occurs for a higher priority task $\tau_{j}$, then the execution of $\tau_{i}$ is preempted, and will resume when the execution of $\tau_{j}$ has ended, as well as all other releases of tasks with a higher priority than $\tau_{i}$ that have taken place in the meantime.

A schedule is an assignment of the tasks to the processor. A schedule can be defined as an integer step function $\sigma: \mathbb{R} \rightarrow\{0,1, \ldots, n\}$, where $\sigma(t)=i$ with $i>0$ means that task $\tau_{i}$ is being executed at time $t$, while $\sigma(t)=0$ means that the processor is idle. More specifically, we define $\sigma(t)$ as a right-continuous and piece-wise constant function, i.e. $\sigma$ partitions the timeline in a set of non-empty, right semi-open

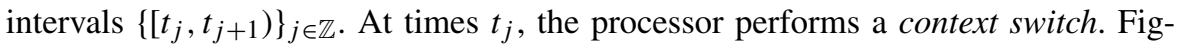
ure 1 shows an example of the execution of a set $\mathcal{T}$ of three periodic tasks and the corresponding value of the schedule $\sigma(t)$.

Each task $\tau_{i}$ is characterized by a (release) period $T_{i} \in \mathbb{R}^{+}$, a computation time $C_{i} \in \mathbb{R}^{+}$, a (relative) deadline $D_{i} \in \mathbb{R}^{+}$, where $C_{i} \leq \min \left(D_{i}, T_{i}\right)$, and a phasing $\varphi_{i} \in \mathbb{R}^{+} \cup\{0\}$. In this paper, we assume arbitrary deadlines, i.e. the deadline of a task may exceed its period. An activation (or release) time is a time at which a task $\tau_{i}$ becomes ready for execution. A release of a task is also termed a job. The first job of task $\tau_{i}$ is released at time $\varphi_{i}$ and is referred to as job zero. The release of job $k$ of $\tau_{i}$ therefore takes place at time $a_{i k}=\varphi_{i}+k T_{i}, k \in \mathbb{N}$. The (absolute) deadline of job $k$ of $\tau_{i}$ takes place at $d_{i k}=a_{i k}+D_{i}$. The begin time $b_{i k}$ and finalization (or

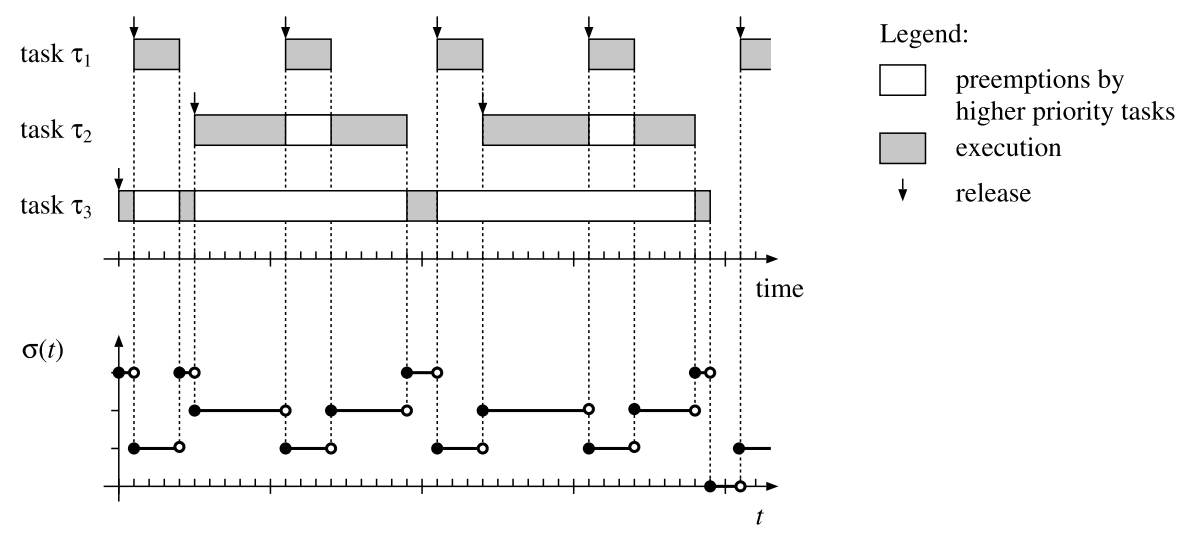

Fig. 1 An example of the execution of a set $\mathcal{T}$ of three independent periodic tasks $\tau_{1}, \tau_{2}$, and $\tau_{3}$, where task $\tau_{1}$ has highest priority, and task $\tau_{3}$ has lowest priority, and the corresponding value of $\sigma(t)$ 

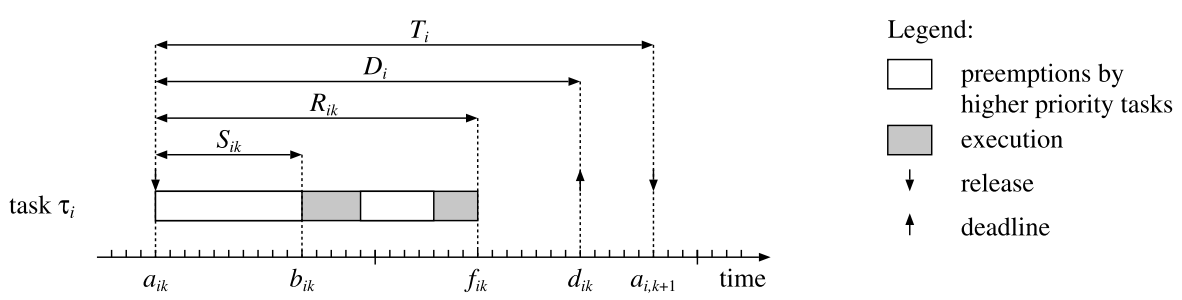

Fig. 2 Basic model for task $\tau_{i}$

completion) time $f_{i k}$ of job $k$ of $\tau_{i}$ is the time at which $\tau_{i}$ actually starts and ends the execution of that job, respectively. The set of phasings $\varphi_{i}$ is termed the phasing $\varphi$ of the task set $\mathcal{T}$.

The active (or response) interval of job $k$ of $\tau_{i}$ is defined as the time span between the activation time of that job and its finalization time, i.e. $\left[a_{i k}, f_{i k}\right)$. The response time $R_{i k}$ of job $k$ of $\tau_{i}$ is defined as the length of its active interval, i.e. $R_{i k}=f_{i k}-a_{i k}$. Similarly, the begin (or start) interval is defined as $\left[a_{i k}, b_{i k}\right.$ ), and the start time $S_{i k}$ as $S_{i k}=b_{i k}-a_{i k}$. Figure 2 illustrates the above basic notions for an example job of task $\tau_{i}$.

The worst-case response time $W R_{i}$ and the worst-case start time of a task $\tau_{i}$ are the largest response time and the largest start time of any of its jobs, respectively, i.e.

$$
\begin{aligned}
W R_{i} & =\sup _{\varphi, k} R_{i k}(\varphi), \\
W S_{i} & =\sup _{\varphi, k} S_{i k}(\varphi) .
\end{aligned}
$$

Note that the response time $R_{i k}$ and the start time $S_{i k}$ have been parameterized in these equations to denote their dependency on the phasing $\varphi$.

In many cases, we are not interested in the worst-case response time of a task for a particular computation time, but in the value as a function of the computation time $C \in \mathbb{R}^{+}$. We will therefore use a functional notation when needed, e.g. $W R_{i}(C)$. A critical instant of a task is defined to be an (hypothetical) instant that leads to the worst-case response time for that task. Typically, such an instant is described as a point in time with particular properties. As an example, a critical instant for tasks under FPPS is given by a point in time for which all tasks have a simultaneous release.

We assume that we do not have control over the phasing $\varphi$, for instance since the tasks are released by external events, so we assume that any arbitrary phasing may occur. This assumption is common in real-time scheduling literature (Joseph and Pandya 1986; Klein et al. 1993; Liu and Layland 1973). We also adopt other standard basic assumptions (Liu and Layland 1973), i.e. tasks are ready to run at the start of each period and do no suspend themselves, tasks will be preempted instantaneously when a higher priority task becomes ready to run, a job of task $\tau_{i}$ does not start before its previous job is completed, and the overhead of context switching and task scheduling is ignored. Finally, we assume that the deadlines are hard, i.e. each job of a task must be completed at or before its deadline. Hence, a set $\mathcal{T}$ of $n$ periodic tasks 
can be scheduled if and only if

$$
W R_{i} \leq D_{i}
$$

for all $i=1, \ldots, n$. For notational convenience, we assume that the tasks are given in order of decreasing priority, i.e. task $\tau_{1}$ has highest priority and task $\tau_{n}$ has lowest priority.

The (processor) utilization factor $U$ is the fraction of the processor time spent on the execution of the task set (Liu and Layland 1973). The fraction of processor time spent on executing task $\tau_{i}$ is $C_{i} / T_{i}$, and is termed the utilization factor $U_{i}^{\tau}$ of task $\tau_{i}$, i.e.

$$
U_{i}^{\tau}=\frac{C_{i}}{T_{i}} .
$$

The cumulative utilization factor $U_{i}$ for tasks $\tau_{1}$ till $\tau_{i}$ is the fraction of processor time spent on executing these tasks, and is given by

$$
U_{i}=\sum_{j \leq i} U_{j}^{\tau}
$$

Therefore, $U$ is equal to the cumulative utilization factor $U_{n}$ for $n$ tasks:

$$
U=U_{n}=\sum_{j \leq n} U_{j}^{\tau}=\sum_{j \leq n} \frac{C_{j}}{T_{j}} .
$$

In Liu and Layland (1973), the following necessary condition is determined for the schedulability of a set $\mathcal{T}$ of $n$ periodic tasks under any scheduling algorithm:

$$
U \leq 1
$$

Unless explicitly stated otherwise, we assume in this document that task sets satisfy this condition.

\subsection{Refined model for FPDS}

For FPDS, we need to refine our basic model of Sect. 2.1. Each job of task $\tau_{i}$ is now assumed to consist of a sequence of $m_{i}$ subjobs. The $k$ th subjob of $\tau_{i}$ is characterized by a computation time $C_{i k} \in \mathbb{R}^{+}$, where $C_{i}=\sum_{k=1}^{m_{i}} C_{i k}$. For convenience, we will use the term $F_{i}$ to denote the computation time $C_{i, m_{i}}$ of the final subjob of $\tau_{i}$, i.e.

$$
F_{i}=C_{i, m_{i}}
$$

We assume that subjobs are non-preemptable. Hence, a task can only be preempted at subjob boundaries, i.e. at so-called preemption points. A task can therefore defer the preemption and execution of a higher priority task. We will use the term blocking of a task $\tau_{i}$ to denote the time that the execution of $\tau_{i}$ is deferred by lower priority tasks. Note that when $m_{i}=1$ for all $i$, we have FPNS as special case. 


\subsection{Concluding remarks}

In this document, we will use the superscript $\mathrm{P}$ to denote FPPS, e.g. $W R_{i}^{\mathrm{P}}$ denotes the worst-case response time of task $\tau_{i}$ under FPPS and arbitrary phasing. Similarly, we will use the superscripts D and N to denote FPDS and FPNS, respectively.

In our basic model for FPPS, we introduced notions for points in time with a subscript identifying a task and optionally a job of that task, e.g. $a_{i k}$ is the absolute release time of job $k$ of task $\tau_{i}$. We will need similar notions that are expressed relative to a particular moment in time, e.g. the relative release time of the first job of a task at or after time $t_{s}$. We will therefore also use relative versions of the notions, where relative can refer to the identification of the job and/or to a particular moment in time, depending on the notion. As an example, let $\phi_{i}(t)$ denote the earliest absolute activation of a job of task $\tau_{i}$ at or after time $t$, i.e.

$$
\phi_{i}(t)=\varphi_{i}+\left(\left\lceil\frac{t-\varphi_{i}}{T_{i}}\right\rceil\right)^{+} \cdot T_{i} .
$$

Here, the notation $x^{+}$stands for $\max (x, 0)$, which is used to indicate that there are no releases of $\tau_{i}$ before time $\varphi_{i}$. Because $\varphi_{i} \geq 0$, the term $\left(\left\lceil\frac{t-\varphi_{i}}{T_{i}}\right\rceil\right)^{+}$is equal to the number of releases of $\tau_{i}$ in $[0, t)$. Given $\phi_{i}(t)$, the relative phasing $\varphi_{i}(t)$ is given by $\varphi_{i}(t)=\phi_{i}(t)-t$. The release of job $k$ of task $\tau_{i}$ relative to $t$ takes place at the relative activation time $a_{i k}(t)=\varphi_{i}(t)+k T_{i}, k \in \mathbb{N}$. For $a_{i k}(t)$, both the identification of the job and the time are therefore relative to $t$. Similarly, the notions relative begin time $b_{i k}(t)$ and relative finalization time $f_{i k}(t)$ denote a time relative to $t$ and concern the job $k$ of task $\tau_{i}$ relative to $t$. For the relative response time $R_{i k}(t)$, only the identification of the job is relative to $t$. We will use abbreviated representations for the relative notions using a prime $\left(^{\prime}\right)$ when the particular moment in time is clear from the context. As an example, in a context concerning a particular moment $t_{s}$, the relative activation time $a_{i k}^{\prime}$ denotes $a_{i k}\left(t_{s}\right)$.

\section{Recapitulation of worst-case analysis for FPPS}

For the analysis under FPPS, we only consider cases where the deadlines of tasks are less than or equal to the respective periods. For illustration purposes, we will use a set $\mathcal{T}_{1}$ of two independent periodic tasks $\tau_{1}$ and $\tau_{2}$ with characteristics as given in Table 1.

Figure 3 shows an example of the execution of the tasks $\tau_{1}$ and $\tau_{2}$ under FPPS. Note that even an infinitesimal increase of the computation time of either task $\tau_{1}$ or $\tau_{2}$ will immediately cause the job of task $\tau_{2}$ released at time 0 to miss its deadline at time 7 .

Table 1 Task characteristics of $\mathcal{T}_{1}$

\begin{tabular}{lll}
\hline & $T_{i}=D_{i}$ & $C_{i}$ \\
\hline$\tau_{1}$ & 5 & 2 \\
$\tau_{2}$ & 7 & 3 \\
\hline
\end{tabular}




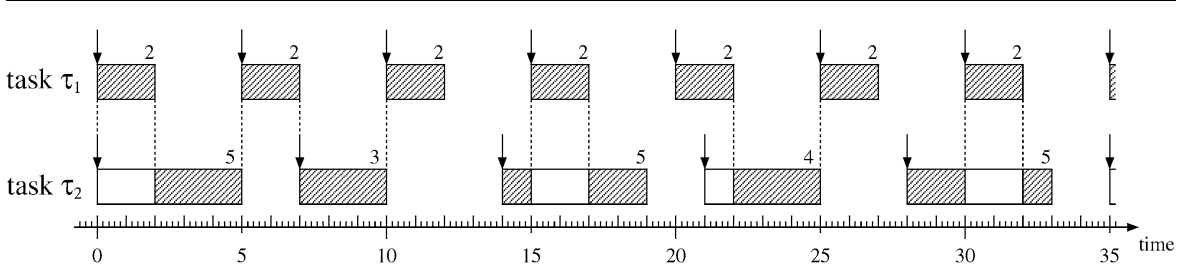

Fig. 3 Timeline for $\mathcal{T}_{1}$ under FPPS with a simultaneous release of both tasks at time zero. The numbers to the top right corner of the boxes denote the response times of the respective releases

\subsection{Worst-case response times}

This section presents theorems for the notion of critical instant and to determine worst-case response times of tasks. Although these theorems are taken from Bril (2004), most of these results were already known; see for example Audsley et al. (1991), Joseph and Pandya (1986), Liu and Layland (1973). Auxiliary lemmas on which the proofs of these theorems and theorems in subsequent sections are based are included in the Appendix.

Theorem 1 (Bril 2004, Theorem 4.1) In order to have a maximal response time for an execution $k$ of task $\tau_{i}$, i.e. to have $f_{i k}-a_{i k}=W R_{i}$, we may assume without loss of generality that the phasing $\varphi$ is such that $\varphi_{j}=a_{i k}$ for all $j<i$. In other words, the phasing of the tasks' release times is such that the release of the considered execution of $\tau_{i}$ coincides with the simultaneous release for all higher priority tasks. This latter point in time is called a critical instant for task $\tau_{i}$.

Given this theorem, we conclude that time 0 in Fig. 3 is a critical instant for both task $\tau_{1}$ and $\tau_{2}$. From this figure, we therefore derive that the worst-case response times of tasks $\tau_{1}$ and $\tau_{2}$ are 2 and 5, respectively. The next theorems can be used to determine the worst-case response times analytically.

Theorem 2 (Bril 2004, Theorem 4.2) ${ }^{1}$ The worst-case response time WR $R_{i}$ of a task $\tau_{i}$ is given by the smallest $x \in \mathbb{R}^{+}$that satisfies the following equation, provided that $x$ is at most $T_{i}$ :

$$
x=C_{i}+\sum_{j<i}\left\lceil\frac{x}{T_{j}}\right\rceil C_{j} .
$$

Theorem 3 (Bril 2004, Theorem 4.3) ${ }^{1}$ The worst-case response time WR $R_{i}$ of task $\tau_{i}$ can be found by the following iterative procedure:

$$
W R_{i}^{(0)}=C_{i},
$$

\footnotetext{
${ }^{1}$ The theorems in Bril (2004, Chap. 4) are based on worst-case computation times of tasks. Because our basic model is based on fixed computation times, we replaced $W C_{i}$ and $W C_{j}$ in the original equation by $C_{i}$ and $C_{j}$, respectively. We consider worst-case computation times in Sect. 8.5.
} 


$$
W R_{i}^{(l+1)}=C_{i}+\sum_{j<i}\left\lceil\frac{W R_{i}^{(l)}}{T_{j}}\right\rceil C_{j}, \quad l=0,1, \ldots
$$

The procedure is stopped when the same value is found for two successive iterations of $l$, or when the deadline $D_{i}$ is exceeded.

\subsection{Worst-case occupied times}

In Fig. 3, task $\tau_{2}$ is preempted at time 15 due to a release of task $\tau_{1}$, and resumes its execution at time 17. The span of time from a task $\tau$ 's release till the moment in time that $\tau$ can start its execution or resume its execution after completion of a computation time $C$ is termed occupied time. The worst-case occupied time (WO) of a task $\tau$ is the largest occupied time of any of its jobs. In Bril (2004), it has been shown that the worst-case occupied time can be described in terms of the worst-case response time by taking a limit from above, ${ }^{2}$ i.e.

$$
W O_{i}(C)=\lim _{x \downarrow C} W R_{i}(x)
$$

Considering Fig. 3, we derive that worst-case occupied times $\mathrm{WO}_{2}(0)$ and $\mathrm{WO}_{2}(3)$ of task $\tau_{2}$ are equal to 2 and 7, respectively. Note that the worst-case occupied time $W O_{2}(0)$ of task $\tau_{2}$ is the longest possible span of time from the release of $\tau_{2}$ till the moment in time that $\tau_{2}$ can start its execution, i.e. $W O_{2}(0)$ of $\tau_{2}$ is the worstcase start time $W S_{2}$ of $\tau_{2}$. Hence, unlike worst-case response times, worst-case occupied times are also defined for a computation time equal to zero. Further note that $W R_{2}\left(C_{2}\right)=5<W O_{2}\left(C_{2}\right)=7$. The next theorems can be used to determine the worst-case occupied times analytically.

Theorem 4 (Bril 2004, Theorem 4.4) ${ }^{1}$ When the smallest positive solution of (9) for a computation time $C_{i}^{\prime}$ is at most $D_{i}$, the worst-case occupied time $W O_{i}$ of a task $\tau_{i}$ for a computation time $C_{i} \in\left[0, C_{i}^{\prime}\right]$ is given by the smallest non-negative $x \in \mathbb{R}$ that satisfies

$$
x=C_{i}+\sum_{j<i}\left(\left\lfloor\frac{x}{T_{j}}\right\rfloor+1\right) C_{j} .
$$

Theorem 5 (Bril 2004, Theorem 4.5)1 The worst-case occupied time $W_{i}$ of task $\tau_{i}$ can be found by the following iterative procedure.

$$
W O_{i}^{(0)}= \begin{cases}\sum_{j<i} C_{j} & \text { for } C_{i}=0, \\ W R_{i} & \text { for } C_{i}>0 ;\end{cases}
$$

\footnotetext{
${ }^{2} \mathrm{~A}$ limit taken from above is sometimes also denoted as $\lim _{x \rightarrow C^{+}}$; see Weisstein (2003). When a function $f(x)$ is defined for a value $x=x_{0}$ and the limit from above (or right-hand limit) of $f(x)$ in $x_{0}$ is equal to $f\left(x_{0}\right), f$ is termed right (-hand) continuous at $x=x_{0}$.
} 


$$
W O_{i}^{(l+1)}=C_{i}+\sum_{j<i}\left(\left\lfloor\frac{W O_{i}^{(l)}}{T_{j}}\right\rfloor+1\right) C_{j}, \quad l=0,1, \ldots
$$

The procedure is stopped when the same value is found for two successive iterations of $l$.

\subsection{Concluding remarks}

The proof of Theorem 4 derives (13) by starting from (12) and subsequently using Lemma 16.

Similarly to (12), we can express $W R_{i}$ in terms of $W O_{i}$ by taking a limit from below, ${ }^{3}$ i.e.

$$
W R_{i}(C)=\lim _{x \uparrow C} W O_{i}(x)
$$

The next two equations express that $W R_{i}(C)$ and $W O_{i}(C)$ are left-continuous and right-continuous, respectively.

$$
\begin{aligned}
W R_{i}(C) & =\lim _{x \uparrow C} W R_{i}(x), \\
W O_{i}(C) & =\lim _{x \downarrow C} W O_{i}(x) .
\end{aligned}
$$

Lemmas related to these latter three equations can be found in the Appendix.

\section{Existing response time analysis for FPDS refuted}

We first recapitulate existing response time analysis under FPDS. Next, we show that the existing analysis is pessimistic. We subsequently give examples refuting the analysis, i.e. examples that show that the existing analysis is optimistic.

\subsection{Recapitulation of existing worst-case response time analysis for FPDS}

In this section, we recapitulate existing worst-case response time analysis for FPDS with arbitrary phasing and deadlines within periods as described in Burns (1994), Burns and Wellings (1997). We include a recapitulation of the analysis for FPNS as presented in Tindell et al. (1994). The main reason for including the latter is that it looks different from the analysis for FPDS and is a basis for the analysis of controller area network (CAN).

\footnotetext{
${ }^{3} \mathrm{~A}$ limit taken from below is sometimes also denoted as $\lim _{x \rightarrow C^{-}}$; see Weisstein (2003). When a function $f(x)$ is defined for a value $x=x_{0}$ and the limit from below (or left-hand limit) of $f(x)$ in $x_{0}$ is equal to $f\left(x_{0}\right), f$ is termed left (-hand) continuous at $x=x_{0}$.
} 


\subsubsection{Existing analysis for FPDS}

The non-preemptive nature of subjobs may cause blocking of a task by at most one lower priority task under FPDS. Moreover, a task can be blocked by at most one subjob of a lower priority task. The maximum blocking $B_{i}^{\mathrm{D}} \in \mathbb{R}^{+} \cup\{0\}$ of task $\tau_{i}$ by a lower priority task is therefore equal to the longest computation time of any subjob of a task with a priority lower than task $\tau_{i}$. This blocking time is given by

$$
B_{i}^{\mathrm{D}}= \begin{cases}\max _{j>i} \max _{1 \leq k \leq m_{j}} C_{j, k} & \text { for } i<n, \\ 0 & \text { for } i=n .\end{cases}
$$

Strictly spoken, this blocking time is a supremum (and not a maximum) for all tasks, except for the lowest priority task, i.e. that value cannot be assumed for $i<n$.

The worst-case response time $\widetilde{W R}_{i}^{\mathrm{D}}$ of a task $\tau_{i}$ under FPDS, arbitrary phasing, and deadlines less than or equal to periods, as presented in Burns (1994) and Burns and Wellings (1997), is given by

$$
\widetilde{W R}_{i}^{\mathrm{D}}=W R_{i}^{\mathrm{P}}\left(B_{i}^{\mathrm{D}}+C_{i}-\left(F_{i}-\Delta\right)\right)+\left(F_{i}-\Delta\right),
$$

where $W R_{i}^{\mathrm{P}}$ denotes the worst-case response time of $\tau_{i}$ under FPPS. According to Burns and Wellings (1997), $\Delta$ is an arbitrary small positive value needed to ensure that the final subjob has actually started. Hence, when task $\tau_{i}$ has consumed $C_{i}-$ $\left(F_{i}-\Delta\right)$, the final subjob has (just) started.

\subsubsection{Existing analysis for FPNS}

In this section, we first recapitulate the update of Joseph and Pandya (1986) given in Tindell et al. (1994) to take account of tasks being non-preemptive. Next, we show that the update is very similar to the analysis for FPDS as given by (20).

The non-preemptive nature of tasks may cause blocking of a task by at most one lower priority task. The maximum blocking $B_{i}^{\mathrm{N}} \in \mathbb{R}^{+} \cup\{0\}$ of task $\tau_{i}$ by a lower priority task is equal to the longest computation time of a task with a priority lower than task $\tau_{i}$, i.e.

$$
B_{i}^{\mathrm{N}}= \begin{cases}\max _{j>i} C_{j} & \text { for } i<n, \\ 0 & \text { for } i=n .\end{cases}
$$

Similarly to $B_{i}^{\mathrm{D}}, B_{i}^{\mathrm{N}}$ is a supremum for all tasks, except for the lowest priority task, i.e. that value cannot be assumed for $i<n$.

The worst-case response time $\widetilde{W R}_{i}^{\mathrm{N}}$ is given by

$$
\widetilde{W R}_{i}^{\mathrm{N}}=w_{i}+C_{i},
$$

where $w_{i}$ is the smallest $x \in \mathbb{R}^{+}$that satisfies

$$
x=B_{i}^{\mathrm{N}}+\sum_{j<i}\left\lceil\frac{x+\tau_{\text {res }}}{T_{j}}\right\rceil C_{j} .
$$


In this latter equation, $\tau_{\text {res }}$ is the resolution with which time is measured. To calculate $w_{i}$, an iterative procedure based on recurrence relationships can be used. An appropriate initial value of this procedure is $w_{i}^{(0)}=B_{i}^{\mathrm{N}}+\sum_{j<i} C_{j}$.

We now show that these results for FPNS are similar to the existing analysis for FPDS. To this end, we substitute $w_{i}=w_{i}^{\prime}-\tau_{\text {res }}, x=x^{\prime}-\tau_{\text {res }}$, and $\tau_{\text {res }}=\Delta$ in (22) and (23). Hence, the worst-case response time $\widetilde{W R}_{i}^{\mathrm{N}}$ is given by

$$
\widetilde{W R}_{i}^{\mathrm{N}}=w_{i}^{\prime}+\left(C_{i}-\Delta\right),
$$

where $w_{i}^{\prime}$ is the smallest $x^{\prime} \in \mathbb{R}^{+}$that satisfies

$$
x^{\prime}=B_{i}^{\mathrm{N}}+\Delta+\sum_{j<i}\left\lceil\frac{x^{\prime}}{T_{j}}\right\rceil C_{j} .
$$

Reusing the results for FPPS, we therefore get

$$
\widetilde{W R}_{i}^{\mathrm{N}}=W R_{i}^{\mathrm{P}}\left(B_{i}^{\mathrm{N}}+\Delta\right)+\left(C_{i}-\Delta\right) .
$$

Because we have $F_{i}=C_{i}$ and $B_{i}^{\mathrm{D}}=B_{i}^{\mathrm{N}}$ for FPNS, (24) for FPNS is similar to (20) for FPDS. There is an aspect requiring further attention, however. In particular, (20) is based on an arbitrary small positive value $\Delta$ whereas the analysis for FPNS is based on the resolution $\tau_{\text {res }}$ with which time is measured. We will return to this issue in Sect. 8.3.

\subsection{Existing analysis is pessimistic}

Consider the set $\mathcal{T}_{2}$ consisting of three tasks with characteristics as described in Table 2. Based on (20) we derive

$$
\begin{aligned}
\widetilde{W R}_{2}^{\mathrm{D}} & =W R_{2}^{\mathrm{P}}\left(B_{2}^{\mathrm{D}}+C_{2}-\left(F_{2}-\Delta\right)\right)+\left(F_{2}-\Delta\right) \\
& =W R_{2}^{\mathrm{P}}(2+3-(2-\Delta))+(2-\Delta) \\
& =W R_{2}^{\mathrm{P}}(3+\Delta)+(2-\Delta)=7+\Delta+(2-\Delta)=9 .
\end{aligned}
$$

However, the existing analysis does not take into account that $\tau_{i}$ can only be blocked by a subjob of a lower priority task if that subjob starts before the simultaneous release of $\tau_{i}$ and all tasks with a higher priority than $\tau_{i}$. This aspect can be taken into account in the analysis by replacing $B_{i}^{\mathrm{D}}$ in $(20)$ by $\left(B_{i}^{\mathrm{D}}-\Delta\right)^{+}$. The notation $x^{+}$stands for $\max \{x, 0\}$, which is used to indicate that the blocking time can not become negative

Table 2 Task characteristics of $\mathcal{T}_{2}$

\begin{tabular}{rrrl}
\hline & $T_{i}$ & $D_{i}$ & $C_{i}$ \\
\hline$\tau_{1}$ & 5 & 4 & 2 \\
$\tau_{2}$ & 7 & 7 & $1+2$ \\
$\tau_{3}$ & 30 & 30 & $2+2$ \\
\hline
\end{tabular}


Fig. 4 Timeline for $\mathcal{T}_{2}$ under FPDS with a release of tasks $\tau_{1}$ and $\tau_{2}$ at time $t=1$ and a release of task $\tau_{3}$ at time $t=1-\Delta$

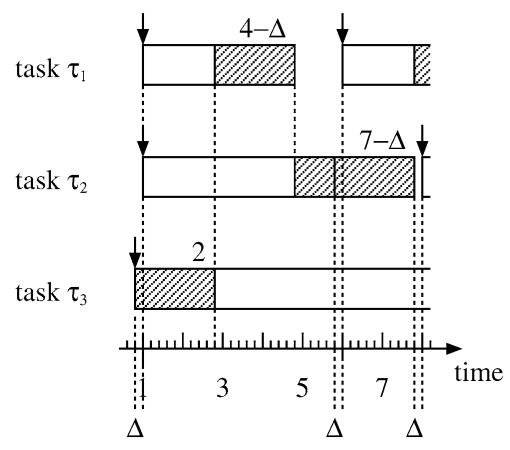

for the lowest priority task. The worst-case response time of $\tau_{2}$ now becomes $7-\Delta$, as illustrated in Fig. 4. For $\Delta \downarrow 0$, we therefore find a supremum (and not a maximum) equal to 7 for the worst-case response time of $\tau_{2}$. As a result, the existing analysis is pessimistic.

\subsection{Existing analysis is optimistic}

We will give three examples illustrating that the existing analysis is optimistic. For all three examples, deadlines are equal to periods, i.e. $D_{i}=T_{i}$. The first section shows an obvious example, i.e. an example with a utilization factor $U>1$. The second section shows an example with $U<1$. The third section shows an example with $U=1$.

For all three examples, the task set consists of just two tasks. For such task sets, the worst-case response time analysis under FPDS presented in Burns (1994, 2001), Burns and Wellings (1997) and in Bril et al. (2004) is very similar. In particular, the worst-case response time $\widetilde{W R}_{2}^{\mathrm{D}}$ of task $\tau_{2}$ is determined by looking at the response time of the first job of task $\tau_{2}$ upon a simultaneous release with task $\tau_{1}$. However, the worst-case response time of task $\tau_{2}$ is not assumed for the first job for all three examples.

\subsubsection{An example with $U>1$}

An example refuting the worst-case response time analysis is given in Table 3. Note that the utilization factor $U$ of this set of tasks $\mathcal{T}_{3}$ is given by $U=\frac{2}{5}+\frac{4.5}{7}>1$. Hence, the task set is not schedulable. Based on (20), we derive

$$
\begin{aligned}
\widetilde{W R}_{2}^{\mathrm{D}} & =W R_{2}^{\mathrm{P}}\left(B_{2}+C_{2}-\left(F_{2}-\Delta\right)\right)+\left(F_{2}-\Delta\right) \\
& =W R_{2}^{\mathrm{P}}(0+4.5-(3-\Delta))+(3-\Delta) \\
& =W R_{2}^{\mathrm{P}}(1.5+\Delta)+(3-\Delta)=3.5+\Delta+(3-\Delta)=6.5 .
\end{aligned}
$$

This value corresponds with the response time of the first job of task $\tau_{2}$ upon a simultaneous release with task $\tau_{1}$, as illustrated in Fig. 5. However, the same figure also illustrates that the second job of $\tau_{2}$ misses its deadline. Stated in other words, the existing worst-case response time analysis is optimistic. 


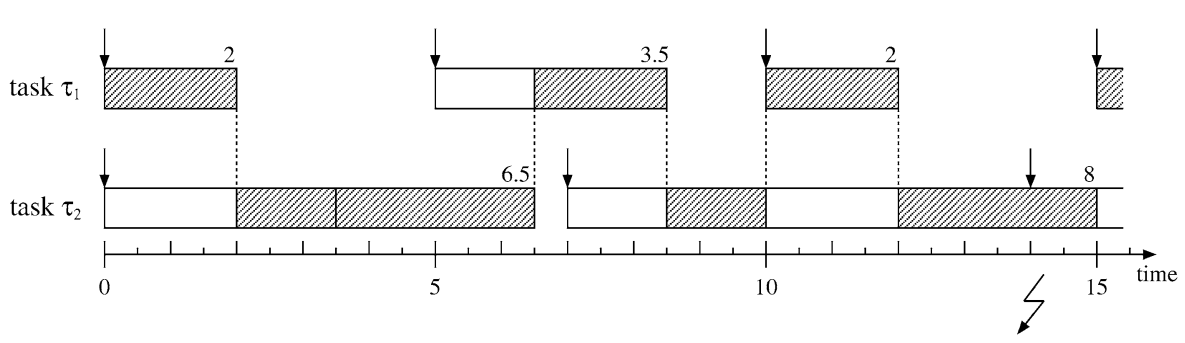

Fig. 5 Timeline for $\mathcal{T}_{3}$ under FPDS with a simultaneous release of both tasks at time zero

Table 3 Task characteristics of $\mathcal{T}_{3}$

\begin{tabular}{lll}
\hline & $T_{i}=D_{i}$ & $C_{i}$ \\
\hline$\tau_{1}$ & 5 & 2 \\
$\tau_{2}$ & 7 & $1.5+3$ \\
\hline
\end{tabular}

Table 4 Task characteristics of $\mathcal{T}_{4}$

\begin{tabular}{lll}
\hline & $T_{i}=D_{i}$ & $C_{i}$ \\
\hline$\tau_{1}$ & 5 & 2 \\
$\tau_{2}$ & 7 & $2+2.1$ \\
\hline
\end{tabular}

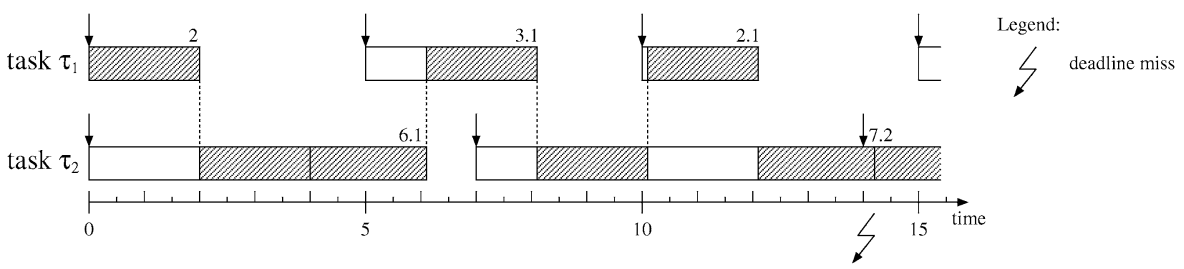

Fig. 6 Timeline for $\mathcal{T}_{4}$ under FPDS with a simultaneous release of all tasks at time zero

\subsubsection{An example with $U<1$}

Another example refuting the worst-case response time analysis is given in Table 4. Note that the utilization factor $U$ of this set of tasks $\mathcal{T}_{4}$ is given by $U=\frac{2}{5}+\frac{4.1}{7}<1$. Hence, the task set could be schedulable. Applying (20) yields $\widetilde{W R}{ }_{2}^{\mathrm{D}}=6.1$, which corresponds with the response time of the first job of task $\tau_{2}$ upon a simultaneous release with task $\tau_{1}$; see Fig. 6. However, the same figure also illustrates that the second job of task $\tau_{2}$ misses its deadline.

\subsubsection{An example with $U=1$}

Consider task set $\mathcal{T}_{5}$ given in Table 5. The utilization factor $U$ of this set of tasks is given by $U=\frac{2}{5}+\frac{4.2}{7}=1$. The task set is not schedulable by FPPS, as we showed in Sect. 3 that the task set is only schedulable when $C_{2}$ is at most 3 . Figure 7 shows a timeline with the executions of these two tasks under FPDS with a simultaneous 


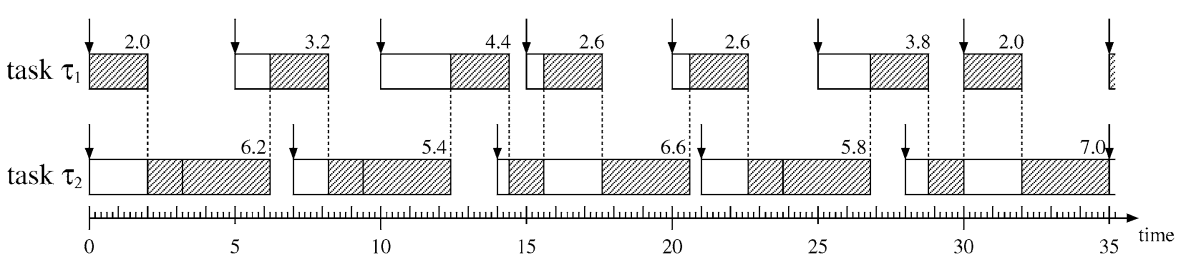

Fig. 7 Timeline for $\mathcal{T}_{5}$ under FPDS with a simultaneous release of all tasks at time zero

Table 5 Task characteristics of $\mathcal{T}_{5}$

Table 6 Task characteristics of $\mathcal{T}_{6}$

\begin{tabular}{lll}
\hline & $T_{i}=D_{i}$ & $C_{i}$ \\
\hline$\tau_{1}$ & 5 & 2 \\
$\tau_{2}$ & 7 & $1.2+3$ \\
\hline
\end{tabular}

\begin{tabular}{lll}
\hline & $T_{i}$ & $C_{i}$ \\
\hline$\tau_{1}$ & 5 & 2 \\
$\tau_{2}$ & 7 & 1.2 \\
$\tau_{3}$ & 7 & 3 \\
\hline
\end{tabular}

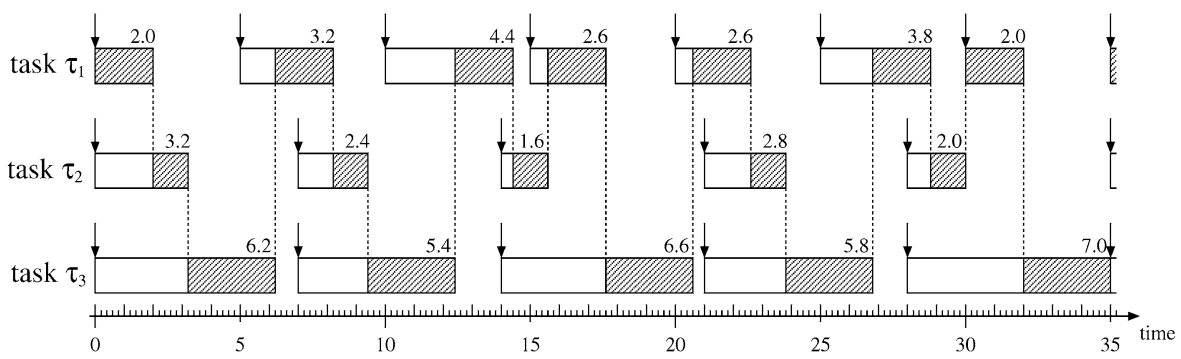

Fig. 8 Timeline for $\mathcal{T}_{6}$ under FPNS with a simultaneous release of all tasks at time zero. The numbers to the top right corner of the boxes denote the response times of the respective releases

release at time zero in an interval of length 35 , i.e. equal to the hyperperiod of the tasks. Applying (20) yields $\widetilde{W R} \mathrm{D}_{2}^{\mathrm{D}}=6.2$, which corresponds with the response time of the first job of task $\tau_{2}$ in Fig. 7. However, the response time of the 5th job of task $\tau_{2}$ is equal to 7 , illustrating once again that the existing analysis is too optimistic. Nevertheless, the task set is schedulable under FPDS for this phasing.

Now, consider task set $\mathcal{T}_{6}$ given in Table 6 , which is similar to task set $\mathcal{T}_{5}$ given in Table 5, except for the fact that rather than having a second subjob for task $\tau_{2}$ it has a task $\tau_{3}$. Figure 8 shows a timeline with the executions of these three tasks under FPNS with a simultaneous release at time zero in an interval of length 35 , i.e. equal to the hyperperiod of the tasks. Applying (20) yields $\widetilde{W R} R_{3}^{\mathrm{D}}=6.2$, which corresponds to the response time of the first job of task $\tau_{3}$ in Fig. 8. However, the response time of 
the 5 th job of task $\tau_{3}$ is equal to 7 , illustrating once again that the existing analysis is too optimistic. Nevertheless, the task set is schedulable under FPNS for this phasing.

\subsection{Concluding remark}

We have shown that we cannot restrict ourselves to the response time of the first job of a task when determining the worst-case response time of that task under FPDS. The reason for this is that the final subjob of a task $\tau_{i}$ can defer the execution of higher priority tasks, which can potentially give rise to higher interference for subsequent jobs of task $\tau_{i}$. This problem can therefore arise for all tasks, except for the highest priority task. González Harbour et al. (1991) identified the same influence of jobs of a task for relative deadlines at most equal to periods in the context of FPPS of periodic tasks with varying execution priority.

Considering Fig. 7, we see that every job of task $\tau_{2}$ in the interval $[0,26.8)$ defers the execution of a job of task $\tau_{1}$. Moreover, that deferred job of task $\tau_{1}$ subsequently gives rise to additional interference for the next job of task $\tau_{2}$. This situation ends when the job of $\tau_{2}$ is started at time $t=28$, i.e. the 5 th job of $\tau_{2}$ does not defer the execution of a job of $\tau_{1}$. Viewed in a different way, we may state that the active intervals of the jobs of tasks $\tau_{1}$ and $\tau_{2}$ overlap in the interval $[0,35)$. Note that this overlapping starts at time $t=0$ and ends at time $t=35$, and we therefore term this interval $[0,35)$ a level-2 active period. Informally, a level-i active period is a smallest interval that only contains entire active intervals of jobs of task $\tau_{i}$ and jobs of tasks with a higher priority than task $\tau_{i}$. Hence, the active interval of every job of a task $\tau_{i}$ is contained in a level- $i$ active period. To determine the worst-case response time of a task $\tau_{i}$, we therefore only have to consider level- $i$ active periods. However, as illustrated by the examples shown in this section and mentioned above, we cannot restrict ourselves to the response time of the first job of a task $\tau_{i}$ when determining the worst-case response time of that task under FPDS. Instead, we have to consider the response times of all jobs in a level- $i$ active period. In a subsequent section, we will show that it suffices to consider only the response times of jobs in a level- $i$ active period that starts at a so-called $\varepsilon$-critical instant.

\section{Active period}

This section presents a formal definition of a level-i active period, which is based on the notion of pending load, and theorems to determine the length of a level- $i$ active period. As mentioned before, a level- $i$ active period may contain multiple jobs of $\tau_{i}$. We therefore also define the notion of a level-( $\left.i, k\right)$ active period, and present a theorem to determine the length of such a period. Informally, a level- $(i, k)$ active period is a smallest interval that only contains $(k+1)$ entire active intervals of jobs 4 of task $\tau_{i}$ and entire active intervals of jobs of tasks with a higher priority than task $\tau_{i}$.

\footnotetext{
${ }^{4}$ The jobs of task $\tau_{i}$ in a level- $(i, k)$ active period are numbered from 0 (zero) to $k$, giving rise to $(k+1)$ jobs.
} 
These notions and theorems form the basis for the worst-case analysis for FPDS in the next section.

We start with the definition of the notion level- $i$ active period in Sect. 5.1. Next, we provide examples of level- $i$ active periods in Sect. 5.2. The length of a level- $i$ active period is the topic of Sect. 5.3. We refine the notion of level- $i$ active period to level- $(i, k)$ active period in Sect. 5.4, and conclude with a theorem to determine its length in Sect. 5.4.3.

\subsection{Level- $i$ active period}

The notion of level- $i$ active period is defined in terms of the notion of pending load, which on its turn is defined in terms of the notion of active job.

\subsubsection{Active job and pending load}

Definition $1 \mathrm{~A}$ job $k$ of a task $\tau_{i}$ is active at time $t$ if and only if $t \in\left[a_{i k}, f_{i k}\right)$, where $a_{i k}$ and $f_{i k}$ are the activation time and the finalization time of that job, respectively.

As described in Sect. 2.1, the active interval of job $k$ of task $\tau_{i}$ is defined as the time span between the activation time of that job and its completion, i.e. $\left[a_{i k}, f_{i k}\right)$. We now define the notion of pending load in terms of active job, and derive properties for the pending load.

Definition 2 The pending load $P_{i}^{\tau}(t)$ is the amount of processing at time $t$ that still needs to be performed for the active jobs of task $\tau_{i}$ that are released before time $t$, i.e.

$$
P_{i}^{\tau}(t)=\left(\left\lceil\frac{t-\varphi_{i}}{T_{i}}\right\rceil\right)^{+} \cdot C_{i}-\int_{0}^{t} \sigma_{i}^{\tau}\left(t^{\prime}\right) d t^{\prime},
$$

where

$$
\sigma_{i}^{\tau}(t)= \begin{cases}1 & \text { if task } \tau_{i} \text { is being executed at time } t, \text { i.e. } \sigma(t)=i \\ 0 & \text { otherwise }\end{cases}
$$

The term $\left(\left\lceil\frac{t-\varphi_{i}}{T_{i}}\right\rceil\right)^{+} \cdot C_{i}$ in (25) is equal to the amount of processing that needs to be performed due to releases of task $\tau_{i}$ in $[0, t)$. The term $\int_{0}^{t} \sigma_{i}^{\tau}\left(t^{\prime}\right) d t^{\prime}$ is equal to the amount of processing that has been performed for $\tau_{i}$. The right-hand side of (25) is therefore equal to the amount of processing at time $t$ due to releases of jobs of task $\tau_{i}$ before $t$ that still needs to be performed.

We subsequently define the notions of (cumulative) pending load $P_{i}(t)$ and (processor) pending load $P(t)$.

Definition 3 The (cumulative) pending load $P_{i}(t)$ is the amount of processing at time $t$ that still needs to be performed for the active jobs of tasks $\tau_{j}$ with $j \leq i$ that are released before time $t$, i.e.

$$
P_{i}(t)=\sum_{j \leq i} P_{j}^{\tau}(t)=\sum_{j \leq i}\left(\left\lceil\frac{t-\varphi_{j}}{T_{j}}\right\rceil\right)^{+} \cdot C_{j}-\int_{0}^{t} \sigma_{i}\left(t^{\prime}\right) d t^{\prime},
$$


where

$$
\sigma_{i}(t)=\sum_{j \leq i} \sigma_{j}^{\tau}(t)= \begin{cases}1 & \text { if } \sigma(t) \in\{1, \ldots, i\} \\ 0 & \text { otherwise }\end{cases}
$$

Definition 4 The (processor) pending load $P(t)$ is the amount of processing at time $t$ that still needs to be performed for the active jobs of all tasks that are released before time $t$, i.e.

$$
P(t)=P_{n}(t)
$$

Corollary 1 The order in which the tasks $\tau_{j}$ with $j \leq i$ are executed is immaterial for the cumulative pending load $P_{i}$.

For $i<n$, the cumulative pending load $P_{i}$ depends on blocking due to a lower priority task. As an example, let $P_{i}\left(t_{s}\right)=0$, then $P_{i}(t)=C_{k}$ for all $t \in\left(t_{s}, t_{s}^{\prime}\right)$ under FPDS if the following three conditions hold:

- A task $\tau_{k}$ with $s \leq i$ is released at time $t_{s}$.

- No other releases of $\tau_{j}$ for $j \leq i$ take place in $\left[t_{s}, t_{s}^{\prime}\right)$.

- A subjob of a lower priority task is executing at time $t_{s}$ and blocks task $\tau_{k}$ during $\left[t_{s}, t_{s}^{\prime}\right)$ due to the non-preemptive nature of the subjob.

Because blocking due to a lower priority task does not play a role for the (processor) pending load, $P(t)$ only depends on the activations of tasks.

Corollary 2 The (processor) pending load $P(t)$ is independent of the scheduling algorithm, provided that the algorithm is non-idling.

Note that the notions of pending load, cumulative pending load, and processor pending load are independent of relative deadlines of tasks.

\subsubsection{Definition of a level-i active period}

We now define the notion of level- $i$ active period in terms of the pending load $P_{i}(t)$.

Definition 5 A level- $i$ active period is an interval $\left[t_{s}, t_{e}\right)$ with the following three properties:

1. $P_{i}\left(t_{s}\right)=0$;

2. $P_{i}\left(t_{e}\right)=0$;

3. $P_{i}(t)>0$ for all $t \in\left(t_{s}, t_{e}\right)$.

Let the blocking time $B_{i}\left(t_{s}\right)$ of a level- $i$ active period that starts at time $t_{s}$ be defined as the length of the (optionally empty) initial interval during which the tasks $\tau_{j}$ with $j \leq i$ are blocked by a subjob of a task with a lower priority. Note that $B_{n}\left(t_{s}\right)=0$ and $0 \leq B_{i}\left(t_{s}\right)<B_{i}^{\mathrm{D}}$ for $i<n$. 
Lemma 1 If a level-i active period starts at time $t_{s}$ and ends at time $t_{e}$, then the following properties hold:

(i) Tasks $\tau_{j}$ with $j \leq i$ are continuously executing in $\left[t_{s}, t_{e}\right.$ ), except for an (optionally empty) initial interval $\left[t_{s}, t_{s}+B_{i}\left(t_{s}\right)\right)$ during which the tasks are blocked by a lower priority task.

(ii) The length $L_{i}\left(t_{s}\right)$ of that level-i active period is at least $B_{i}\left(t_{s}\right)+C_{k}$, where a task $\tau_{k}$ with $k \leq i$ is released at time $t_{s}$.

(iii) The order in which the tasks $\tau_{j}$ with $j \leq i$ are executed is immaterial for the length $L_{i}\left(t_{s}\right)$.

Proof (i) This property follows immediately from the non-preemptive nature of subjobs and the assumptions for fixed-priority scheduling.

(ii) By definition, $P_{i}\left(t_{s}\right)=0$. Because the tasks $\tau_{j}$ with $j \leq i$ are blocked in the (optionally empty) initial interval $\left[t_{s}, t_{s}+B_{i}\left(t_{s}\right)\right.$ ), and the level- $i$ active period contains at least the active interval of task $\tau_{k}$, the length $L_{i}\left(t_{s}\right)$ of that level- $i$ active period is at least $B_{i}\left(t_{s}\right)+C_{k}$.

(iii) This property follows immediately from the definition of a level- $i$ active period and Corollary 1.

From this definition of the level- $i$ active period in terms of the pending load $P_{i}(t)$, we draw the following conclusion.

Corollary 3 The level-n active period is independent of the scheduling algorithm, provided that the algorithm is non-idling.

Note that the notion of level- $i$ active period is independent of relative deadlines of tasks. Further note that a level- $i$ active period may, but need not, contain activations of task $\tau_{i}$. In the sequel, we will call a level- $i$ active period that contains an activation of task $\tau_{i}$ a proper level- $i$ active period. Similarly, we call a level- $i$ active period that does not contain an activation of $\tau_{i}$ an improper level- $i$ active period. Unless explicitly stated otherwise, we use the phrase 'level- $i$ active period' to denote a proper level- $i$ active period in the remainder of this document.

\subsection{Examples}

We will now consider two examples, one for FPPS based on the timeline in Fig. 3 for $\mathcal{T}_{1}$ and one for FPDS based on the timeline in Fig. 7 for $\mathcal{T}_{5}$.

Consider Fig. 9, with a timeline for $\mathcal{T}_{1}$ under FPPS, pending loads $P_{1}(t), P_{2}^{\tau}(t)$, and $P_{2}(t)$, and level- $i$ active periods. Note that $P_{1}(t)$ is equal to $P_{1}^{\tau}(t)$ by definition. From the graph for $P_{1}(t)$, we find that the interval $[0,35)$ contains seven level-1 active periods, corresponding with the seven activations of task $\tau_{1}$, i.e. $[0,5)$, $[5,7),[10,12),[15,17),[20,22),[25,27)$, and $[30,32)$. The horizontal line fragments in the graph for $P_{2}^{\tau}(t)$ are caused by the fact that $\tau_{2}$ is preempted by a job of task $\tau_{1}$. From the graph for the pending load $P_{2}(t)$, we find that the interval $[0,35)$ contains eight level-2 active periods, i.e. $[0,5),[5,7),[7,10),[10,12),[14,19)$, $[20,25),[25,27)$, and $[28,33)$. From these eight level-2 active periods, $[0,5),[7,10)$, 

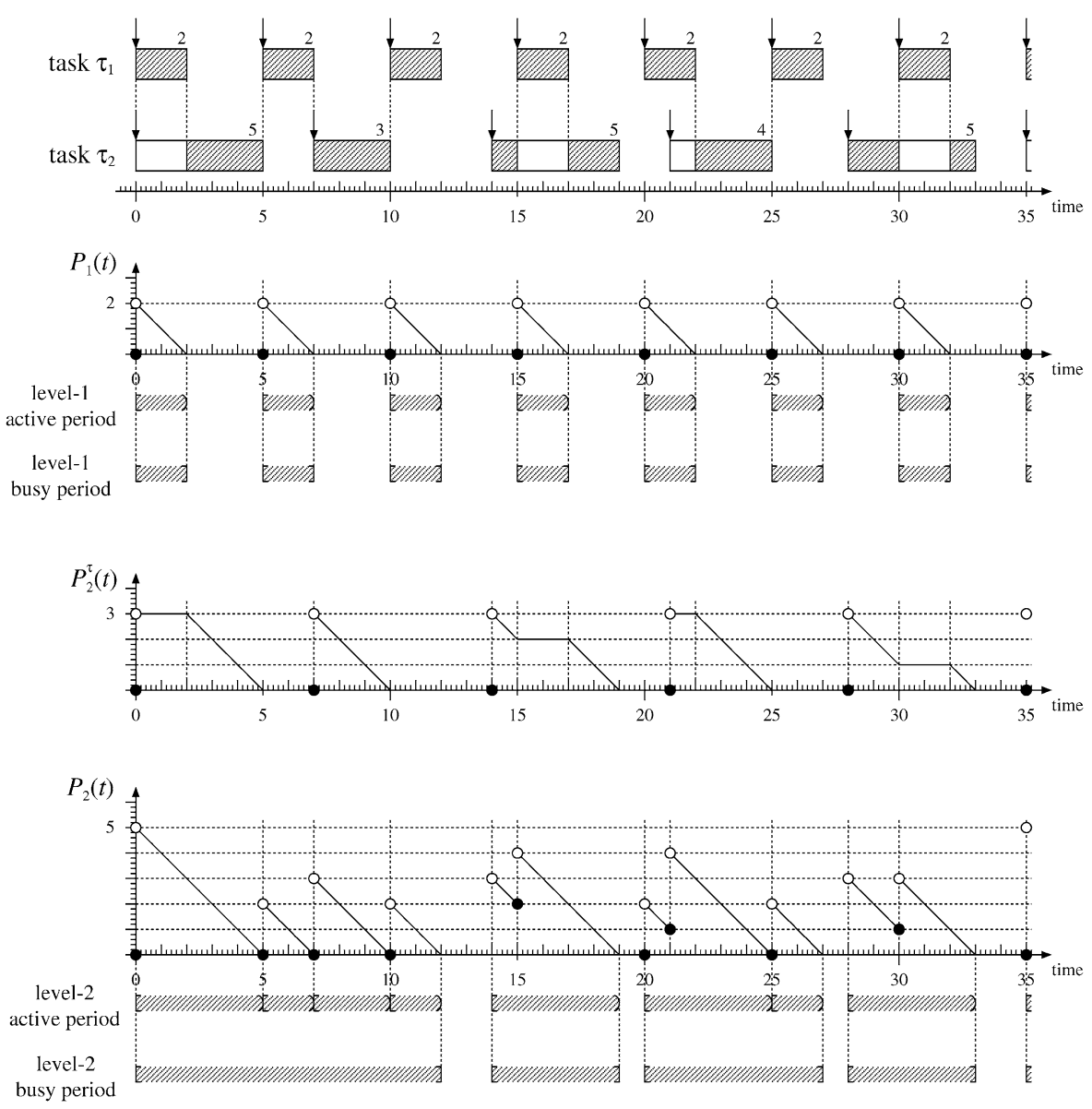

Fig. 9 Timeline for $\mathcal{T}_{1}$ under FPPS, pending loads $P_{1}(t), P_{2}^{\tau}(t)$, and $P_{2}(t)$, and level- $i$ active periods and level- $i$ busy periods. From the eight level- 2 active periods in the interval $[0,35)$, five are proper, i.e. $[0,5),[7,10),[14,19),[20,25)$, and $[28,33)$ contain activations of task $\tau_{2}$. The other three are improper, i.e. $[5,7),[10,12)$, and $[25,27)$

$[14,19),[20,25)$, and $[28,33)$ are proper, i.e. contain activations of task $\tau_{2}$, and $[5,7),[10,12)$, and $[25,27)$ are improper. As mentioned before, the level-2 active period only depends on the activations of $\tau_{1}$ and $\tau_{2}$, and is independent of the scheduling algorithm.

Consider Fig. 10, with a timeline for $\mathcal{T}_{5}$ under FPDS, pending loads $P_{1}(t), P_{2}^{\tau}(t)$, and $P_{2}(t)$, and level- $i$ active periods. From the graph for $P_{1}(t)$, we find that the interval $[0,35)$ contains seven level- 1 active periods, corresponding with the seven activations of task $\tau_{1}$, i.e. [0, 2), [5, 8.2), [10, 14.4), [15, 17.6), [20, 22.6), [25, 28.8), and $[30,32)$. The horizontal line fragments in the graph for $P_{1}(t)$ are caused by the fact that $\tau_{1}$ is blocked by a subjob of task $\tau_{2}$. From the graph for the pending load $P_{2}(t)$, we find that the interval $[0,35)$ contains a single level- 2 active period, i.e. $[0,35)$. 

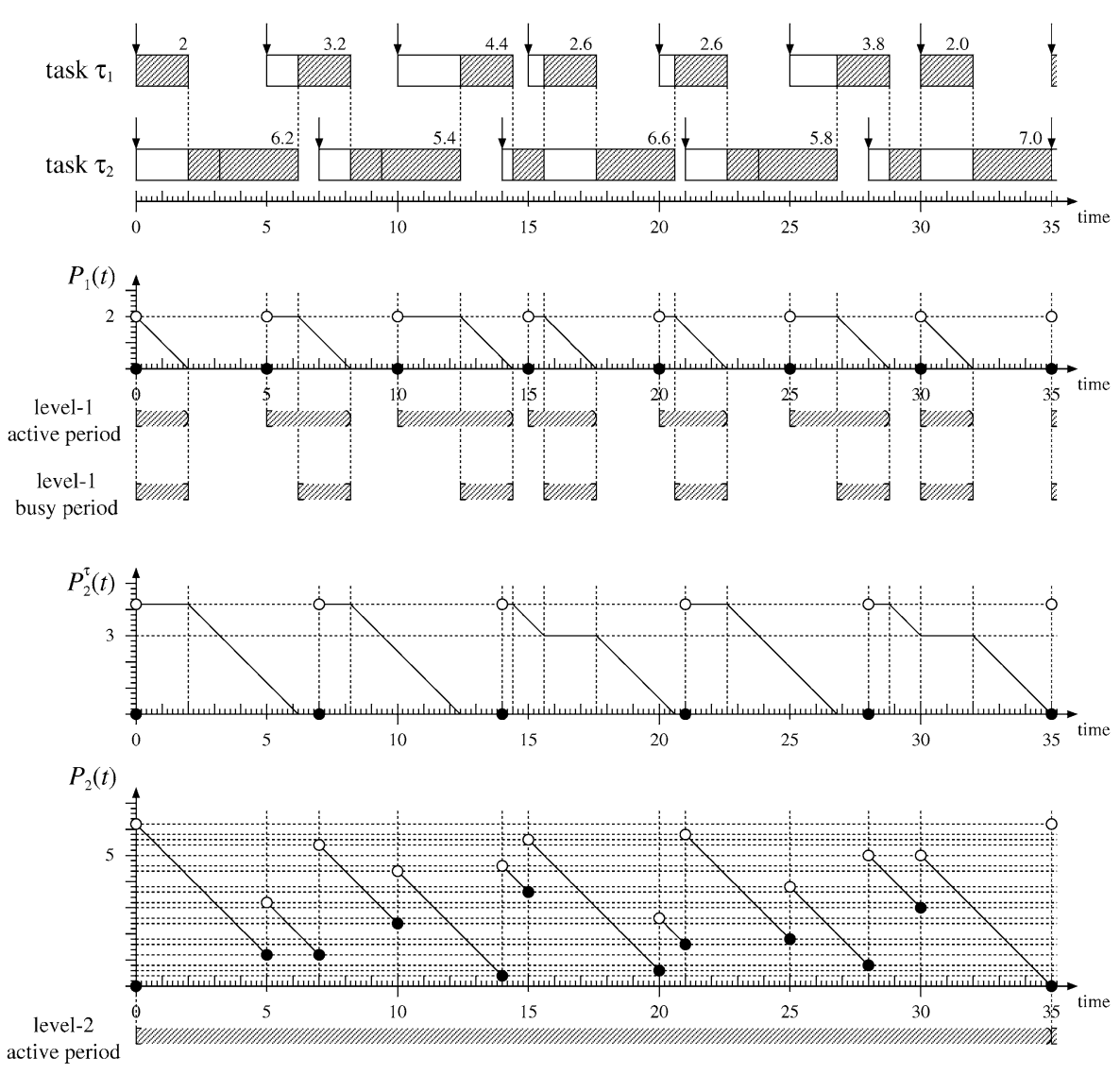

level-2

busy period

Fig. 10 Timeline for $\mathcal{T}_{5}$ under FPDS, pending loads $P_{1}(t), P_{2}^{\tau}(t)$, and $P_{2}(t)$, and level- $i$ active periods and level- $i$ busy periods

\subsection{Length of a level- $i$ active period}

This section presents three theorems for the length of a level- $i$ active period. A first theorem presents a recursive equation for the length of a level- $i$ active period. A next theorem states that under the following assumption a level- $i$ active period that starts will also end.

Assumption 1 Either $U<1$ or $U \leq 1$ and the least common multiple (lcm) of the periods of the tasks of $\mathcal{T}$ exists.

Hence, the assumption is a sufficient condition to guarantee that a level- $i$ active period will end when it starts. Because we assume $\varphi_{i} \geq 0$ for all $i \leq n, P_{i}(0)=0$ for all $i \leq n$. We therefore conclude that, when Assumption 1 holds, the timeline consists of a sequence of level- $i$ active periods, optionally preceded by and separated by idle- 
periods. A final theorem provides an iterative procedure to determine the length of a level- $i$ active period.

\subsubsection{A recursive equation}

Theorem 6 The length $L_{i}\left(t_{s}\right)$ of a level-i active period that starts at time $t_{s}$ is found for the smallest $x \in \mathbb{R}^{+}$that satisfies the following equation

$$
x=B_{i}\left(t_{s}\right)+\sum_{j \leq i}\left(\left\lceil\frac{x-\varphi_{j}\left(t_{s}\right)}{T_{j}}\right\rceil\right)^{+} \cdot C_{j} .
$$

Proof Because the level- $i$ active period starts at time $t_{s}, P_{i}\left(t_{s}\right)=0$ by definition. Now assume the level- $i$ active period under consideration ends at time $t_{e}$. Hence, time $t_{e}$ is the smallest $t$ larger than $t_{s}$ for which $P_{i}(t)=0$, and the length $L_{i}\left(t_{s}\right)$ of the active period becomes $t_{e}-t_{s}$. We now derive (28) from $P_{i}\left(t_{e}\right)=0$ :

$$
\begin{aligned}
P_{i}\left(t_{e}\right) & =\{(26)\} \quad \sum_{j \leq i}\left(\left\lceil\frac{t_{e}-\varphi_{j}}{T_{j}}\right\rceil\right)^{+} \cdot C_{j}-\int_{0}^{t_{e}} \sigma_{i}(t) d t \\
& =P_{i}\left(t_{s}\right)+\sum_{j \leq i}\left(\left\lceil\frac{t_{e}-\left(t_{s}+\varphi_{j}\left(t_{s}\right)\right)}{T_{j}}\right]\right)^{+} \cdot C_{j}-\int_{t_{s}}^{t_{e}} \sigma_{i}(t) d t \\
& =\left\{P_{i}\left(t_{s}\right)=0\right\} \sum_{j \leq i}\left(\left\lceil\frac{t_{e}-\left(t_{s}+\varphi_{j}\left(t_{s}\right)\right)}{T_{j}}\right\rceil\right)^{+} \cdot C_{j}-\int_{t_{s}}^{t_{e}} \sigma_{i}(t) d t \\
& =0 .
\end{aligned}
$$

From Lemma 1, we derive that the lower priority task is executing in $\left[t_{s}, t_{s}+B_{i}\left(t_{s}\right)\right)$, and only tasks $\tau_{j}$ with $j \leq i$ are executing in $\left[t_{s}+B_{i}\left(t_{s}\right), t_{e}\right)$. Hence, we conclude that

$$
\int_{t_{s}}^{t_{e}} \sigma_{i}(t) d t=t_{e}-\left(t_{s}+B_{i}\left(t_{s}\right)\right)
$$

Substituting this result in the former equation, we get

$$
t_{e}-\left(t_{s}+B_{i}\left(t_{s}\right)\right)=\sum_{j \leq i}\left(\left\lceil\frac{t_{e}-\left(t_{s}+\varphi_{j}\left(t_{s}\right)\right)}{T_{j}}\right\rceil\right)^{+} \cdot C_{j},
$$

and by subsequently substituting $t_{e}=x+t_{s}$, we get (28). Because time $t_{e}$ is the smallest $t$ (larger than $t_{s}$ ) for which $P_{i}(t)=0, x=t_{e}-t_{s}$ is the smallest value in $\mathbb{R}^{+}$ that satisfies (28), which proves the theorem.

\subsubsection{End of a level-i active period}

We now present a theorem which states that there exist positive solutions for the recursive equation (28) if Assumption 1 holds. To that end, we will use Lemma 4.3 from Bril (2004) (see Lemma 15 in the Appendix), and first prove two lemmas. 
Lemma 2 There exists a positive solution for the recursive equation (28) for the length of the level-i active period if $U_{i}<1$.

Proof We will prove that the condition $U_{i}<1$ is sufficient by means of Lemma 4.3 of Bril (2004). Let $f$ be defined as

$$
f(x)=B_{i}\left(t_{s}\right)+\sum_{j \leq i}\left(\left\lceil\frac{x-\varphi_{j}\left(t_{s}\right)}{T_{j}}\right\rceil\right)^{+} \cdot C_{j} .
$$

We choose $a=\min _{l \leq i} \frac{C_{l}}{2}$, hence

$$
f(a)=f\left(\min _{l \leq i} \frac{C_{l}}{2}\right)=B_{i}\left(t_{s}\right)+\sum_{j \leq i}\left(\left\lceil\frac{\min _{l \leq i} \frac{C_{l}}{2}-\varphi_{j}\left(t_{s}\right)}{T_{j}}\right\rceil\right)^{+} \cdot C_{j}
$$

By definition, there is at least one task that is released at the start of the level- $i$ active period. Let task $\tau_{k}$ with $k \leq i$ be released at time $t_{s}$, i.e. $\varphi_{k}\left(t_{s}\right)=0$. We now get

$$
f(a) \geq B_{i}\left(t_{s}\right)+\left\lceil\frac{\min _{l \leq i} \frac{C_{l}}{2}}{T_{k}}\right\rceil C_{k}=B_{i}\left(t_{s}\right)+C_{k}>\min _{l \leq i} \frac{C_{l}}{2}=a,
$$

hence $f(a)>a$. In order to choose an appropriate $b$, we make the following derivation.

$$
f(x) \leq B_{i}\left(t_{s}\right)+\sum_{j \leq i}\left\lceil\frac{x}{T_{j}}\right\rceil C_{j}<B_{i}\left(t_{s}\right)+\sum_{j \leq i}\left(\frac{x}{T_{j}}+1\right) C_{j}=B_{i}\left(t_{s}\right)+x U_{i}+\sum_{j \leq i} C_{j} .
$$

As $U_{i}<1$, the relation

$$
x \geq B_{i}\left(t_{s}\right)+x U_{i}+\sum_{j \leq i} C_{j}
$$

holds for

$$
x \geq \frac{B_{i}\left(t_{s}\right)+\sum_{j \leq i} C_{j}}{1-U_{i}} .
$$

We now choose

$$
b=\frac{B_{i}\left(t_{s}\right)+\sum_{j \leq i} C_{j}}{1-U_{i}},
$$

and therefore get $b>f(b)$. Now the conditions for Lemma 15 hold, i.e. the function $f(x)$ is defined and strictly non-decreasing in an interval $[a, b]$ with $f(a)>a$ and $f(b)<b$. Hence, there exists an

$$
x \in\left(\min _{l \leq i} \frac{C_{l}}{2}, \frac{B_{i}\left(t_{s}\right)+\sum_{j \leq i} C_{j}}{\left(1-U_{i}\right)}\right)
$$

such that $x=f(x)$. 
Lemma 3 There exists a positive solution for the recursive equation (28) for the length of the level-n active period if $U=1$ and the least common multiple of the periods of $\mathcal{T}$ exists.

Proof We first observe that $B_{n}\left(t_{s}\right)=0$ for the level- $n$ active period, i.e. the lowest priority task is never blocked. Next, we distinguish two complementary cases, a first case with $\varphi_{i}\left(t_{s}\right)=0$ for all $i$ and a second case where this does not hold. We prove the lemma by considering both cases separately.

For the first case, we prove that for $B_{n}\left(t_{s}\right)=0$ and $\varphi_{i}\left(t_{s}\right)=0$ for all $i$ the value $x=\operatorname{lcm}\left(T_{1}, \ldots, T_{n}\right)$ is a solution of (28). For these values of $B_{n}\left(t_{s}\right)$ and $\varphi_{i}\left(t_{s}\right),(28)$ simplifies to

$$
x=\sum_{j \leq n}\left\lceil\frac{x}{T_{j}}\right\rceil C_{j} .
$$

Because $\left\lceil\frac{\operatorname{lcm}\left(T_{1}, \ldots, T_{n}\right)}{T_{j}}\right\rceil C_{j}=\operatorname{lcm}\left(T_{1}, \ldots, T_{n}\right) \frac{C_{j}}{T_{j}}$ and $\sum_{j \leq n} \frac{C_{j}}{T_{j}}=U=1$, we immediately see that $\operatorname{lcm}\left(T_{1}, \ldots, T_{n}\right)$ is a (positive) solution.

For the second case, we prove that the condition ' $U=1$ and the least common multiple of the periods of $\mathcal{T}$ exists' is sufficient by means of Lemma 15 . Let $f$ be defined as

$$
f(x)=\sum_{j \leq n}\left(\left\lceil\frac{x-\varphi_{j}\left(t_{s}\right)}{T_{j}}\right\rceil\right)^{+} \cdot C_{j} .
$$

We choose $a=\min _{j \leq n} C_{j} / 2$. Similar to the proof of Lemma 2, we find $f(a)>a$. In order to choose an appropriate $b$, we make the following derivation.

$$
f(x) \leq \sum_{j \leq n}\left\lceil\frac{x}{T_{j}}\right\rceil C_{j} .
$$

We now consider two disjunct cases for $x=\operatorname{lcm}\left(T_{1}, \ldots, T_{n}\right)$. If $f(x)<\sum_{j \leq n}\left\lceil\frac{x}{T_{j}}\right\rceil C_{j}$, we choose $b=\operatorname{lcm}\left(T_{1}, \ldots, T_{n}\right)$, and therefore get $b>f(b)$. Now the conditions for Lemma 15 hold, i.e. the function $f(x)$ is defined and strictly non-decreasing in an interval $[a, b]$ with $f(a)>a$ and $f(b)<b$. Hence, there exists an $x \in$ $\left(\min _{j \leq n} \frac{C_{j}}{2}, \operatorname{lcm}\left(T_{1}, \ldots, T_{n}\right)\right)$ such that $x=f(x)$. If $f(x)=\sum_{j \leq i}\left\lceil\frac{x}{T_{j}}\right\rceil C_{j}$, we found a (positive) solution and we are also done.

Theorem 7 If Assumption 1 holds, a level-i active period that is started at time $t_{s}$ is guaranteed to end.

Proof The theorem follows immediately from Lemmas 2 and 3.

\subsubsection{An iterative procedure}

The next theorem provides an iterative procedure to determine the length of a level- $i$ active period. 
Theorem 8 Let the level-i active period start with a release of a task $\tau_{k}$ at time $t_{s}$. If Assumption 1 holds, the length $L_{i}\left(t_{s}\right)$ of that level-i active period can be found by the following iterative procedure:

$$
\begin{aligned}
L_{i}^{(0)}\left(t_{s}\right) & =B_{i}\left(t_{s}\right)+C_{k} \\
L_{i}^{(l+1)}\left(t_{s}\right) & =B_{i}\left(t_{s}\right)+\sum_{j \leq i}\left(\left\lceil\frac{L_{i}^{(l)}\left(t_{s}\right)-\varphi_{j}\left(t_{s}\right)}{T_{j}}\right\rceil\right)^{+} \cdot C_{j}, \quad l=0,1, \ldots
\end{aligned}
$$

Proof From Lemmas 2 and 3, we know that there exists a positive solution of (28) when Assumption 1 holds. To prove the theorem, we first prove that the sequence is non-decreasing. Next, we prove that the procedure stops when the length $L_{i}\left(t_{s}\right)$ is reached, i.e. for the smallest solution of (28). To that end, we show that all values in the sequence $L_{i}^{(l)}\left(t_{s}\right)$ are lower bounds on $L_{i}\left(t_{s}\right)$. To show that the procedure terminates, we show that the sequence can only take a finite number of values to reach that solution.

We prove that the sequence is non-decreasing, by induction. To this end,we start by noting that $L_{i}^{(0)}\left(t_{s}\right)=B_{i}\left(t_{s}\right)+C_{k}>0$, and

$$
\begin{aligned}
L_{i}^{(1)}\left(t_{s}\right) & =B_{i}\left(t_{s}\right)+\sum_{j \leq i}\left(\left\lceil\frac{L_{i}^{(0)}\left(t_{s}\right)-\varphi_{j}\left(t_{s}\right)}{T_{j}}\right\rceil\right)^{+} \cdot C_{j} \\
& \geq\left\{\varphi_{s}\left(t_{s}\right)=0\right\} B_{i}\left(t_{s}\right)+C_{k}=L_{i}^{(0)}\left(t_{s}\right) .
\end{aligned}
$$

Next, if $L_{i}^{(l+1)}\left(t_{s}\right) \geq L_{i}^{(l)}\left(t_{s}\right)$, then we can conclude from (30) that also $L_{i}^{(l+2)}\left(t_{s}\right) \geq$ $L_{i}^{(l+1)}\left(t_{s}\right)$, as filling in a higher value in the right-hand side of (30) gives a higher or equal result.

We next prove $L_{i}^{(l)}\left(t_{s}\right) \leq L_{i}\left(t_{s}\right)$, for all $l=0,1, \ldots$, by induction. From Lemma 1 item (ii) we know $L_{i}^{(0)}\left(t_{s}\right)=B_{i}\left(t_{s}\right)+C_{k} \leq L_{i}\left(t_{s}\right)$. Next, if $L_{i}^{(l)}\left(t_{s}\right)$ is a lower bound on $L_{i}\left(t_{s}\right)$, then

$$
\sum_{j \leq i}\left(\left\lceil\frac{L_{i}^{(l)}\left(t_{s}\right)-\varphi_{j}\left(t_{s}\right)}{T_{j}}\right\rceil\right)^{+} \cdot C_{j}
$$

is a lower bound on the amount of processing that needs to be performed due to releases of task $\tau_{i}$ and its higher priority tasks in the interval of length $L_{i}^{(l)}\left(t_{s}\right)$, and hence $L_{i}^{(l+1)}\left(t_{s}\right)$ is also a lower bound on $L_{i}\left(t_{s}\right)$.

Finally, we prove that the sequence can only take on a finite number of values. To this end, we note that $L_{i}^{(l)}\left(t_{s}\right)$ is bounded from below by $B_{i}\left(t_{s}\right)+C_{k}$ and from above by the solution. This means that each factor $\left(\left\lceil\frac{L_{i}^{(l)}\left(t_{s}\right)-\varphi_{j}\left(t_{s}\right)}{T_{j}}\right\rceil\right)^{+}$in (30) can only take on a finite number of values. Combining this for all higher priority tasks $\tau_{j}$, we can conclude that the right-hand side of (30) can only take on a finite number of values. 


\subsection{Level- $(i, k)$ active period}

Similar to a level- $i$ active period, a level- $(i, k)$ active period is defined in terms of the notion pending load. For the definition of a level- $(i, k)$ active period, we first need to refine the notion of pending load. We assume in this section that Assumption 1 holds.

\subsubsection{A refinement of pending load}

Let a level- $i$ active period start at time $t_{s}$. As described above, the length of that period is given by the smallest $x>0$ satisfying (28). Let the length of that period be $L_{i}\left(t_{s}\right)$. The number of jobs $l_{i}\left(t_{s}\right)$ of task $\tau_{i}$ in that period is now given by

$$
l_{i}\left(t_{s}\right)=\left\lceil\frac{L_{i}\left(t_{s}\right)-\varphi_{i}\left(t_{s}\right)}{T_{i}}\right\rceil .
$$

We now refine our notion of pending load $P_{i}(t)$ by considering only the first $k+1 \leq$ $l_{i}\left(t_{s}\right)$ jobs of $\tau_{i}$ in the active period, where $k \in \mathbb{N}$.

Definition 6 The pending load $P_{i k}(t)$ in a level- $i$ active period that started at time $t_{s}<t$ and ends at time $t_{e} \geq t$ is the amount of processing at time $t$ that still needs to be performed for at most the first $k+1 \leq l_{i}\left(t_{s}\right)$ jobs of $\tau_{i}$ and the jobs of tasks $\tau_{j}$ with $j<i$ that are released in $\left[t_{s}, t\right)$, i.e.

$$
\begin{aligned}
P_{i k}(t)= & \min \left(\left(\left\lceil\frac{t-\left(t_{s}+\varphi_{i}\left(t_{s}\right)\right)}{T_{i}}\right\rceil\right)^{+}, k+1\right) \cdot C_{i} \\
& +\sum_{j<i}\left(\left\lceil\frac{t-\left(t_{s}+\varphi_{j}\left(t_{s}\right)\right)}{T_{j}}\right\rceil\right)^{+} \cdot C_{j}-\int_{t_{s}}^{t} \sigma_{i}\left(t^{\prime}\right) d t^{\prime},
\end{aligned}
$$

with $\sigma_{i}(t)$ as defined in Definition 3. At the start of a level- $i$ active period and outside level- $i$ active periods, $P_{i k}(t)$ is equal to zero.

\subsubsection{Definition of a level-( $i, k)$ active period}

Similarly, we refine our notion of level- $i$ active period to level- $(i, k)$ active period.

Definition 7 A level- $(i, k)$ active period is an interval $\left[t_{s}, t_{e}\right)$ with the following three properties:

1. $P_{i k}\left(t_{s}\right)=0$;

2. $P_{i k}\left(t_{e}\right)=0$;

3. $P_{i k}(t)>0$ for $t \in\left(t_{s}, t_{e}\right)$.

\subsubsection{Length of a level-( $i, k)$ active period}

Theorem 9 Let the number of jobs of task $\tau_{i}$ in a level-i active period that starts at time $t_{s}$ be given by $l_{i}\left(t_{s}\right)$. The length $L_{i k}\left(t_{s}\right)$ of that level- $(i, k)$ active period with 
$0 \leq k<l_{i}\left(t_{s}\right)$ is the smallest $x \in \mathbb{R}^{+}$satisfies the following equation

$$
x=B_{i}\left(t_{s}\right)+(k+1) C_{i}+\sum_{j<i}\left(\left\lceil\frac{x-\varphi_{j}\left(t_{s}\right)}{T_{j}}\right\rceil\right)^{+} \cdot C_{j} .
$$

Proof The proof is similar to the proof of Theorem 6.

\section{Worst-case analysis for FPDS}

This section provides theorems for the notions of critical instant and worst-case response times for tasks under FPDS, arbitrary phasing, and arbitrary relative deadlines, and theorems to determine the worst-case response times analytically. We assume in this section that Assumption 1 holds. Moreover, we consider an arbitrary level- $i$ active period with a start at time $t_{s}$. As described in Sect. 2.3, we will use abbreviated representations for the relative notions using a prime $\left(^{\prime}\right)$ to denote the value of these notions relative to time $t_{s}$, e.g. we use $a_{i k}^{\prime}$ to denote $a_{i k}\left(t_{s}\right)$.

\subsection{A critical instant}

Similar to (1), the worst-case response time $W R_{i}^{\mathrm{D}}$ of a task $\tau_{i}$ under FPDS is the largest response time under arbitrary phasing, i.e.

$$
W R_{i}^{\mathrm{D}}=\sup _{\varphi, k} R_{i k}(\varphi)
$$

We can refine this equation by taking blocking of tasks and the notion of level- $i$ active period into account. In particular, we observe that all active intervals of jobs of task $\tau_{i}$ are contained in level- $i$ active periods. Assuming the start of an arbitrary level- $i$ active period at time $t_{s}$, the worst-case response time $W R_{i}^{\mathrm{D}}$ of task $\tau_{i}$ can therefore be described as

$$
W R_{i}^{\mathrm{D}}=\sup _{B_{i}^{\prime}, \varphi_{1}^{\prime}, \ldots, \varphi_{i}^{\prime}} \max _{0 \leq k<l_{i}^{\prime}\left(B_{i}^{\prime}, \varphi_{1}^{\prime}, \ldots, \varphi_{i}^{\prime}\right)} R_{i k}^{\prime}\left(B_{i}^{\prime}, \varphi_{1}^{\prime}, \ldots, \varphi_{i}^{\prime}\right),
$$

where $l_{i}^{\prime}$ is the number of jobs of task $\tau_{i}$ in that level- $i$ active period.

We will now first present a lemma to determine the response time of job $k$ of task $\tau_{i}$ in a level- $i$ active period. We subsequently present a theorem which states that given an infinitesimal time $\varepsilon>0$, the maximum response time of task $\tau_{i}$ is assumed in a level- $i$ active period which starts at an $\varepsilon$-critical instant. A next theorem refines (34).

Lemma 4 The response time $R_{i k}^{\prime}$ of job $k$ of task $\tau_{i}$ in a level-i active period that starts at time $t_{s}$ with $0 \leq k<l_{i}^{\prime}$ and $l_{i}^{\prime}$ the number of jobs of task $\tau_{i}$ in that level- $i$ active period is given by

$$
R_{i k}^{\prime}\left(B_{i}^{\prime}, \varphi_{1}^{\prime}, \ldots, \varphi_{i}^{\prime}\right)=b_{i k, m_{i}}^{\prime}\left(B_{i}^{\prime}, \varphi_{1}^{\prime}, \ldots, \varphi_{i-1}^{\prime}\right)+F_{i}-\left(k T_{i}+\varphi_{i}^{\prime}\right)
$$


where $b_{i k, m_{i}}^{\prime}$ is the relative begin time of the final subjob of job $k$, given by the smallest non-negative $x \in \mathbb{R}$ satisfying

$$
x=B_{i}^{\prime}+(k+1) C_{i}-F_{i}+\sum_{j<i}\left(\left\lfloor\frac{x-\varphi_{j}^{\prime}}{T_{j}}\right\rfloor+1\right)^{+} \cdot C_{j} .
$$

Proof We first look at the relative begin time $b_{i k, m_{i}}^{\prime}$ of the final subjob of that job $k$, and subsequently describe $R_{i k}^{\prime}$ in terms of the relative begin time, the relative activation time $a_{i k}^{\prime}$ and the computation time $F_{i}$ of that final subjob.

The final subjob of job $k$ of task $\tau_{i}$ in the level- $i$ active period that starts at time $t_{s}$ can begin at time $t_{s}+b_{i k, m_{i}}^{\prime}$ when:

- The blocking subjob of the lower priority task has executed $B_{i}^{\prime}$.

- All higher priority tasks that are released in $\left[t_{s}, t_{s}+b_{i k, m_{i}}^{\prime}\right]$ have a completion in that interval.

- All earlier jobs of task $\tau_{i}$ and all earlier subjobs of job $k$ that are released in $\left[t_{s}, t_{s}+\right.$ $\left.b_{i k, m_{i}}^{\prime}\right]$ have a completion in that interval.

Note that the order in which the subjobs in the interval $\left[t_{s}, t_{s}+b_{i k, m_{i}}^{\prime}\right]$ are executed is irrelevant for the begin time of the final subjob of job $k$ of task $\tau_{i}$. Stated in other words, the final subjob of job $k$ of task $\tau_{i}$ can start for the smallest $t_{s}+x \geq t_{s}+$ $\max \left(B_{i}^{\prime}, a_{i k}^{\prime}\right)$ for which $\lim _{t \downarrow t_{s}+x} P_{i k}(t)=F_{i}$. We now derive

$$
\begin{aligned}
\lim _{t \downarrow t_{s}+x} P_{i k}(t)= & \{(32)\} \lim _{t \downarrow t_{s}+x}\left(\min \left(\left(\left[\frac{t-\left(t_{s}+\varphi_{i}^{\prime}\right)}{T_{i}}\right\rceil\right)^{+}, k+1\right) \cdot C_{i}\right. \\
& \left.+\sum_{j<i}\left(\left\lceil\frac{t-\left(t_{s}+\varphi_{j}^{\prime}\right)}{T_{j}}\right\rceil\right)^{+} \cdot C_{j}-\int_{t_{s}}^{t} \sigma_{i}\left(t^{\prime}\right) d t^{\prime}\right) \\
= & \min \left(\left(\lim _{t \downarrow t_{s}+x}\left[\frac{t-\left(t_{s}+\varphi_{i}^{\prime}\right)}{T_{i}}\right\rceil\right)^{+}, k+1\right) \cdot C_{i} \\
& +\sum_{j<i}\left(\lim _{t \downarrow t_{s}+x}\left[\frac{t-\left(t_{s}+\varphi_{j}^{\prime}\right)}{T_{j}}\right]\right)^{+} \cdot C_{j}-\int_{t_{s}}^{t_{s}+x} \sigma_{i}\left(t^{\prime}\right) d t^{\prime} \\
= & \{\operatorname{Lemma} 16\} \min \left(\left(\left\lfloor\frac{x-\varphi_{i}^{\prime}}{T_{i}}\right]^{+}, k\right)^{+}, k\right) \cdot C_{i} \\
& +\sum_{j<i}\left(\left\lfloor\frac{x-\varphi_{j}^{\prime}}{T_{j}}\right\rfloor+1\right)^{+} \cdot C_{j}-\int_{t_{s}}^{t_{s}+x} \sigma_{i}\left(t^{\prime}\right) d t^{\prime} \\
= & \left\{x \geq \max \left(B_{i}^{\prime}, \varphi_{i}^{\prime}+k \cdot T_{i}\right)\right\}(k+1) \cdot C_{i} \\
& +\sum_{j<i}\left(\left\lfloor\frac{x-\varphi_{j}^{\prime}}{T_{j}}\right\rfloor+1\right)^{+} \cdot C_{j}-\left(x-B_{i}^{\prime}\right) \\
= & F_{i} .
\end{aligned}
$$


The relative begin time $b_{i k, m_{i}}^{\prime}\left(B_{i}^{\prime}, \varphi_{1}^{\prime}, \ldots, \varphi_{i-1}^{\prime}\right)$ is therefore the smallest non-negative $x \in \mathbb{R}$ satisfying the following equation:

$$
x=B_{i}^{\prime}+(k+1) C_{i}-F_{i}+\sum_{j<i}\left(\left\lfloor\frac{x-\varphi_{j}^{\prime}}{T_{j}}\right\rfloor+1\right)^{+} \cdot C_{j} .
$$

The relative completion time $f_{i k}^{\prime}$ of job $k$ of $\tau_{i}$ is now given by the relative begin time $b_{i k, m_{i}}^{\prime}$ plus the computation time $F_{i}$, i.e. $f_{i k}^{\prime}=b_{i k, m_{i}}^{\prime}+F_{i}$. The response time $R_{i k}^{\prime}$ of the job $k$ is given by the relative completion time $f_{i k}^{\prime}$ minus the relative activation time $a_{i k}^{\prime}$, i.e.

$$
R_{i k}^{\prime}\left(B_{i}^{\prime}, \varphi_{1}^{\prime}, \ldots, \varphi_{i}^{\prime}\right)=b_{i k, m_{i}}^{\prime}\left(B_{i}^{\prime}, \varphi_{1}^{\prime}, \ldots, \varphi_{i-1}^{\prime}\right)+F_{i}-\left(k T_{i}+\varphi_{i}^{\prime}\right) .
$$

Theorem 10 Given an infinitesimal time $\varepsilon>0$, the maximum response time of task $\tau_{i}$ under FPDS and arbitrary phasing is assumed when the level-i active period is started at an $\varepsilon$-critical instant, i.e. when $\tau_{i}$ has a simultaneous release with all higher priority tasks and a subjob of the lower priority tasks with computation time $B_{i}^{\mathrm{D}}$ starts a time $\varepsilon$ before that simultaneous release.

Proof Let $R_{i}^{\prime}\left(B_{i}^{\prime}, \varphi_{1}^{\prime}, \ldots, \varphi_{i}^{\prime}\right)$ denote $\max _{0 \leq k<l_{i}^{\prime}\left(B_{i}^{\prime}, \varphi_{1}^{\prime}, \ldots, \varphi_{i}^{\prime}\right)} R_{i k}^{\prime}\left(B_{i}^{\prime}, \varphi_{1}^{\prime}, \ldots, \varphi_{i}^{\prime}\right)$. We will prove that $R_{i}^{\prime}\left(B_{i}^{\prime}, \varphi_{1}^{\prime}, \ldots, \varphi_{i}^{\prime}\right)$ assumes a maximum for $\varphi_{j}^{\prime}=0$ with $j \leq i$ and $B_{i}^{\prime}=\left(B_{i}^{\mathrm{D}}-\varepsilon\right)^{+}$. Hence, the maximum is assumed when $\tau_{i}$ has a simultaneous release with all higher priority tasks, and a subjob of a lower priority task with computation time $B_{i}^{\mathrm{D}}$ starts an infinitesimal time $\varepsilon>0$ before that simultaneous release, which proves the theorem.

Based on Theorem 7, i.e. termination of a level- $i$ active period under Assumption 1, we conclude that:

- Only a finite number of jobs need to be considered to determine the worst-case response time of task $\tau_{i}$.

- Every job of $\tau_{i}$ in a level- $i$ active period has a finite response time.

We will now look at the value of the length $L_{i}^{\prime}$ of the level- $i$ active period, the number $l_{i}^{\prime}$ of jobs of task $\tau_{i}$ in the level- $i$ active period, and the response time $R_{i k}^{\prime}$ as a function of the relative phasing $\varphi_{j}^{\prime}$ with $j \leq i$ and the blocking time $B_{i}^{\prime}$. Consider (28) for the length $L_{i}^{\prime}$ of a level- $i$ active period. The term $\left\lceil\frac{x-\varphi_{j}^{\prime}}{T_{j}}\right\rceil$ in that equation is a strictly non-increasing function of $\varphi_{j}^{\prime}$ with $j \leq i$. Because $\varphi_{j}^{\prime} \geq 0$, a maximum of that term is assumed for $\varphi_{j}^{\prime}=0$. Moreover, the right-hand side of (28) is a strictly increasing function of $B_{i}^{\prime}$, and the length $L_{i}^{\prime}$ is therefore also a strictly increasing function of $B_{i}^{\prime}$. The largest value of $L_{i}^{\prime}$ is found for the largest value of $B_{i}^{\prime}$ under consideration, i.e. for $B_{i}^{\prime}=\left(B_{i}^{\mathrm{D}}-\varepsilon\right)^{+}$. As a consequence, $L_{i}^{\prime}$ assumes a maximum for $\varphi_{j}^{\prime}=0$ for all $j \leq i$ and $B_{i}^{\prime}=\left(B_{i}^{\mathrm{D}}-\varepsilon\right)^{+}$.

Given the behavior of $L_{i}^{\prime}$ and (31), we conclude that the number of jobs $l_{i}^{\prime}$ of task $\tau_{i}$ in the level- $i$ active period is a strictly non-increasing function of $\varphi_{j}^{\prime}$ with $j \leq i$ and a strictly non-decreasing function of $B_{i}^{\prime}$. As a consequence, $l_{i}^{\prime}$ assumes a maximum for $\varphi_{j}^{\prime}=0$ for all $j \leq i$ and $B_{i}^{\prime}=\left(B_{i}^{\mathrm{D}}-\varepsilon\right)^{+}$. 
From (35), we conclude that $R_{i k}^{\prime}\left(B_{i}^{\prime}, \varphi_{1}^{\prime}, \ldots, \varphi_{i}^{\prime}\right)$ is a strictly decreasing function of $\varphi_{i}^{\prime}$. Because $\varphi_{i}^{\prime} \geq 0$, a maximum is assumed for $\varphi_{i}^{\prime}=0$. Now consider (36) for the relative begin time $b_{i k, m_{i}}^{\prime}$. The term $\left\lfloor\frac{x-\varphi_{j}}{T_{j}}\right\rfloor$ in that equation is a strictly nonincreasing function of $\varphi_{j}^{\prime}$. Similarly to $\varphi_{i}^{\prime}, \varphi_{j}^{\prime} \geq 0$, a maximum of that term is therefore assumed for $\varphi_{j}^{\prime}=0$. Hence, $b_{i k, m_{i}}^{\prime}\left(B_{i}^{\prime}, 0, \ldots, 0\right)$ dominates $b_{i k, m_{i}}^{\prime}\left(B_{i}^{\prime}, \varphi_{1}^{\prime}, \ldots, \varphi_{i-1}^{\prime}\right)$ for all values of $B_{i}^{\prime}$ and all values of $\varphi_{j}^{\prime}$ with $j<i$. Moreover, the right-hand side of (36) is a strictly increasing function of $B_{i}^{\prime}$, and $b_{i k, m_{i}}^{\prime}\left(B_{i}^{\prime}, 0, \ldots, 0\right)$ is therefore also a strictly increasing function of $B_{i}^{\prime}$. The largest value of $b_{i k, m_{i}}^{\prime}\left(B_{i}^{\prime}, 0, \ldots, 0\right)$ is found for the largest value of $B_{i}^{\prime}$ under consideration, i.e. for $B_{i}^{\prime}=\left(B_{i}^{\mathrm{D}}-\varepsilon\right)^{+}$. As a consequence, $R_{i k}^{\prime}\left(B_{i}^{\prime}, \varphi_{1}^{\prime}, \ldots, \varphi_{i}^{\prime}\right)$ also assumes a maximum for $\varphi_{j}^{\prime}=0$ for all $j \leq i$ and $B_{i}^{\prime}=\left(B_{i}^{\mathrm{D}}-\varepsilon\right)^{+}$.

From the values of $L_{i}^{\prime}, l_{i}^{\prime}$ and $R_{i k}^{\prime}$ as a function of the relative phasing $\varphi_{j}^{\prime}$ with $j \leq i$ and the blocking time $B_{i}^{\prime}$, we conclude that $R_{i}^{\prime}\left(B_{i}^{\prime}, \varphi_{1}^{\prime}, \ldots, \varphi_{i}^{\prime}\right)$ is a strictly nonincreasing function of $\varphi_{1}^{\prime}, \ldots, \varphi_{i-1}^{\prime}$, a strictly decreasing function of $\varphi_{i}^{\prime}$, and a strictly increasing function of $B_{i}^{\prime}$. As a result, $R_{i}^{\prime}\left(B_{i}^{\prime}, \varphi_{1}^{\prime}, \ldots, \varphi_{i}^{\prime}\right)$ assumes a maximum for $\varphi_{j}^{\prime}=0$ with $j \leq i$ and $B_{i}^{\prime}=\left(B_{i}^{\mathrm{D}}-\varepsilon\right)^{+}$, which proves the theorem.

Theorem 11 The worst-case response time WR $R_{i}^{\mathrm{D}}$ of task $\tau_{i}$ under FPDS and arbitrary phasing is given by

$$
W R_{i}^{\mathrm{D}}=\lim _{\varepsilon \downarrow 0} \max _{0 \leq k<l_{i}^{\prime}\left(\left(B_{i}^{\mathrm{D}}-\varepsilon\right)^{+}, 0, \ldots, 0\right)} R_{i k}^{\prime}\left(\left(B_{i}^{\mathrm{D}}-\varepsilon\right)^{+}, 0, \ldots, 0\right) .
$$

Proof Once again, let $R_{i}^{\prime}\left(B_{i}^{\prime}, \varphi_{1}^{\prime}, \ldots, \varphi_{i}^{\prime}\right)$ denote

$$
\max _{0 \leq k<l_{i}^{\prime}\left(\left(B_{i}^{\mathrm{D}}-\varepsilon\right)^{+}, 0, \ldots, 0\right)} R_{i k}^{\prime}\left(\left(B_{i}^{\mathrm{D}}-\varepsilon\right)^{+}, 0, \ldots, 0\right) .
$$

From the proof of Theorem 10, we derive that $R_{i}^{\prime}\left(B_{i}^{\prime}, 0, \ldots, 0\right)$ dominates $R_{i}^{\prime}\left(B_{i}^{\prime}, \varphi_{1}^{\prime}\right.$, $\left.\ldots, \varphi_{i}^{\prime}\right)$ for all values of $B_{i}^{\prime}$ and all values of $\varphi_{j}^{\prime}$ with $j \leq i$, i.e.

$$
W R_{i}^{\mathrm{D}}=\sup _{B_{i}^{\prime}, \varphi_{1}^{\prime}, \ldots, \varphi_{i}^{\prime}} R_{i}^{\prime}\left(B_{i}^{\prime}, \varphi_{1}^{\prime}, \ldots, \varphi_{i}^{\prime}\right)=\sup _{B_{i}^{\prime}} R_{i}^{\prime}\left(B_{i}^{\prime}, 0, \ldots, 0\right) .
$$

Moreover, $R_{i}^{\prime}\left(B_{i}^{\prime}, \varphi_{1}^{\prime}, \ldots, \varphi_{i}^{\prime}\right)$ is a strictly increasing, i.e. monotonic, function of $B_{i}^{\prime}$. Hence,

$$
W R_{i}^{\mathrm{D}}=\sup _{B_{i}^{\prime}} R_{i}^{\prime}\left(B_{i}^{\prime}, 0, \ldots, 0\right)=\lim _{\varepsilon \downarrow 0} R_{i}^{\prime}\left(\left(B_{i}^{\mathrm{D}}-\varepsilon\right)^{+}, 0, \ldots, 0\right),
$$

which proves the theorem.

In the sequel, we will omit trailing zeros in the parameter list, e.g. we write $R_{i k}^{\prime}\left(\left(B_{i}^{\mathrm{D}}-\varepsilon\right)^{+}\right)$when $\varphi_{j}^{\prime}=0$ for $j \leq i$.

From the previous two theorems, we draw the following conclusions. 
Corollary 4 The worst-case response time $W R_{i}^{\mathrm{D}}$ is a supremum (and not a maximum) for all tasks, except for the lowest priority task, i.e. that value cannot be assumed for $i<n$.

Corollary 5 A critical instant is a supremum for all tasks, except for the lowest priority task, i.e. that instant cannot be assumed for $i<n$.

\subsection{Worst-case response times}

The next theorem describes $W R_{i}^{\mathrm{D}}$ in terms of the worst-case response time $W R_{i}^{\mathrm{P}}$ and worst-case occupied time $W O_{i}^{\mathrm{P}}$ under FPPS.

First, we present definitions and prove three lemmas for the worst-case length $W L_{i}^{\mathrm{D}}$ of a level- $i$ active period, the maximum number $w l_{i}^{\mathrm{D}}$ jobs of task $\tau_{i}$ in a level- $i$ active period, and the worst-case response time $W R_{i k}^{\mathrm{D}}$ of job $k$ of task $\tau_{i}$.

Definition 8 The worst-case length $W L_{i}^{\mathrm{D}}$ of level- $i$ active period under FPDS is the largest length of that period under arbitrary phasing, i.e.

$$
W L_{i}^{\mathrm{D}}=\sup _{B_{i}^{\prime}, \varphi_{1}^{\prime}, \ldots, \varphi_{i}^{\prime}} L_{i}^{\prime}\left(B_{i}^{\prime}, \varphi_{1}^{\prime}, \ldots, \varphi_{i}^{\prime}\right) .
$$

Definition 9 The worst-case number $w l_{i}^{\mathrm{D}}$ of jobs of task $\tau_{i}$ in a level- $i$ active period under FPDS is the largest number in that period under arbitrary phasing, i.e.

$$
w l_{i}^{\mathrm{D}}=\sup _{B_{i}^{\prime}, \varphi_{1}^{\prime}, \ldots, \varphi_{i}^{\prime}} l_{i}^{\prime}\left(B_{i}^{\prime}, \varphi_{1}^{\prime}, \ldots, \varphi_{i}^{\prime}\right) .
$$

Definition 10 The worst-case response time $W R_{i k}^{\mathrm{D}}$ of job $k$ of task $\tau_{i}$, with $1 \leq k<$ $w l_{i}^{\mathrm{D}}$, under FPDS is the largest response time of job $k$ of $\tau_{i}$ under arbitrary phasing, i.e.

$$
W R_{i k}^{\mathrm{D}}=\sup _{B_{i}^{\prime}, \varphi_{1}^{\prime}, \ldots, \varphi_{i}^{\prime}} R_{i k}^{\prime}\left(B_{i}^{\prime}, \varphi_{1}^{\prime}, \ldots, \varphi_{i}^{\prime}\right)
$$

Lemma 5 The worst-case length $W L_{i}^{\mathrm{D}}$ of a level-i active period with $i \leq n$ under FPDS is given by the smallest $x \in \mathbb{R}^{+}$that satisfies the following equation

$$
x=B_{i}^{\mathrm{D}}+\sum_{j \leq i}\left\lceil\frac{x}{T_{j}}\right\rceil C_{j} .
$$

Proof The term $\left\lceil\frac{x-\varphi_{j}^{\prime}}{T_{j}}\right\rceil$ in (28) is a strictly non-increasing function of $\varphi_{j}^{\prime}$ with $j \leq i$. Because $\varphi_{j}^{\prime} \geq 0$, a maximum of that term is assumed for $\varphi_{j}^{\prime}=0$. Now let $L_{i}^{\prime}\left(B_{i}^{\prime}\right)$ denote the length of a level- $i$ active period with $i \leq n$ for a simultaneous release of task $\tau_{i}$ with all tasks with a higher priority. Hence, $L_{i}^{\prime}\left(B_{i}^{\prime}\right)$ is the smallest $x \in \mathbb{R}^{+}$ 
satisfying (28) with $\varphi_{j}^{\prime}=0$, i.e. the smallest $x \in \mathbb{R}^{+}$satisfying

$$
x=B_{i}^{\prime}+\sum_{j \leq i}\left\lceil\frac{x}{T_{j}}\right\rceil C_{j} .
$$

We will now consider the cases $i<n$ and $i=n$ separately.

$\{i=n\}$ The lowest priority task is never blocked, therefore $B_{n}^{\mathrm{D}}=0$, and we immediately get (41) by substituting $B_{i}^{\prime}=0$ in (42) for $i=n$.

$\{i<n\}$ The right-hand side of (42) is a strictly increasing function of $B_{i}^{\prime}$, and $L_{i}^{\prime}\left(B_{i}^{\prime}\right)$ is therefore also a strictly increasing function of $B_{i}^{\prime}$. The largest value for $L_{i}^{\prime}\left(B_{i}^{\prime}\right)$ is found for the largest value of $B_{i}^{\prime}<B_{i}^{\mathrm{D}}$. Hence, $W L_{i}^{\mathrm{D}}$ is given by

$$
W L_{i}^{\mathrm{D}}=\lim _{B_{i}^{\prime} \uparrow B_{i}^{\mathrm{D}}} L_{i}^{\prime}\left(B_{i}^{\prime}\right)
$$

Given Lemma 17, we can make the following derivation starting from this equation:

$$
\begin{aligned}
W L_{i}^{\mathrm{D}} & =\{(42)\} \lim _{B_{i}^{\prime} \uparrow B_{i}^{\mathrm{D}}}\left(B_{i}^{\prime}+\sum_{j \leq i}\left\lceil\frac{L_{i}^{\prime}\left(B_{i}^{\prime}\right)}{T_{j}}\right\rceil C_{j}\right) \\
& =B_{i}^{\mathrm{D}}+\sum_{j \leq i} \lim _{B_{i}^{\prime} \uparrow B_{i}^{\mathrm{D}}}\left\lceil\frac{L_{i}^{\prime}\left(B_{i}^{\prime}\right)}{T_{j}}\right\rceil C_{j} \\
& =\left\{\text { Lemma 17\} } B_{i}^{\mathrm{D}}+\sum_{j \leq i}\left\lceil\lim _{B_{i}^{\prime} \uparrow B_{i}^{\mathrm{D}}} \frac{L_{i}^{\prime}\left(B_{i}^{\prime}\right)}{T_{j}}\right\rceil C_{j}\right. \\
& =\{(43)\} B_{i}^{\mathrm{D}}+\sum_{j \leq i}\left\lceil\frac{W L_{i}^{\mathrm{D}}}{T_{j}}\right\rceil C_{j} .
\end{aligned}
$$

Hence, the worst-case length $W L_{i}^{\mathrm{D}}$ is the smallest $x \in \mathbb{R}^{+}$satisfying (41), which proves the lemma.

Because $B_{i}^{\mathrm{D}}$ is a supremum (and not a maximum) for all tasks, except for the lowest priority task, we draw the following conclusion from the previous lemma.

Corollary 6 The worst-case length $W L_{i}^{\mathrm{D}}$ is a supremum (and not a maximum) for all tasks, except for the lowest priority task, i.e. that value cannot be assumed for $i<n$.

Lemma 6 The maximum number $w l_{i}^{\mathrm{D}}$ of jobs of task $\tau_{i}$ in a level-i active period with $i \leq n$ under FPDS is given by

$$
w l_{i}^{\mathrm{D}}=\left\lceil\frac{W L_{i}^{\mathrm{D}}}{T_{i}}\right\rceil .
$$

Proof We first derive (44) and subsequently prove that $w l_{i}^{\mathrm{D}}$ is a maximum. 
As described in the proof of Theorem $10, l_{i}^{\prime}\left(B_{i}^{\prime}\right)$ is a strictly non-decreasing function of the blocking time $B_{i}^{\prime}$. Because $B_{i}^{\mathrm{D}}$ is a supremum that cannot be assumed, the largest value for $l_{i}^{\prime}\left(B_{i}^{\prime}\right)$ is therefore found for the largest value of $B_{i}^{\prime}<B_{i}^{\mathrm{D}}$. Hence, $w l_{i}^{\mathrm{D}}$ is given by

$$
w l_{i}^{\mathrm{D}}=\lim _{B_{i}^{\prime} \uparrow B_{i}^{\mathrm{D}}} l_{i}^{\prime}\left(B_{i}^{\prime}\right)
$$

Because $\frac{L_{i}^{\prime}\left(B_{i}^{\prime}\right)}{T_{i}}$ is a strictly increasing function of $B_{i}^{\prime}$, we can use Lemma 17 in the following derivation

$$
\begin{aligned}
\lim _{B_{i}^{\prime} \uparrow B_{i}^{\mathrm{D}}} l_{i}^{\prime}\left(B_{i}^{\prime}\right) & =\lim _{B_{i}^{\prime} \uparrow B_{i}^{\mathrm{D}}}\left\lceil\frac{L_{i}^{\prime}\left(B_{i}^{\prime}\right)}{T_{i}}\right\rceil \\
& =\{\text { Lemma } 17\}\left\lceil\lim _{B_{i}^{\prime} \uparrow B_{i}^{\mathrm{D}}} \frac{L_{i}^{\prime}\left(B_{i}^{\prime}\right)}{T_{i}}\right\rceil \\
& =\{(43)\}\left\lceil\frac{W L_{i}^{\mathrm{D}}}{T_{i}}\right\rceil .
\end{aligned}
$$

Equation (44) immediately follows from (45) and this latter equation.

The proof that $w l_{i}^{\mathrm{D}}$ is a maximum consists of two steps. We first prove that $l_{i}^{\prime}\left(B_{i}^{\prime}\right)$ is left-continuous in $B_{i}^{\mathrm{D}}$, i.e.

$$
l_{i}^{\prime}\left(B_{i}^{\mathrm{D}}\right)=\lim _{B_{i}^{\prime} \uparrow B_{i}^{\mathrm{D}}} l_{i}^{\prime}\left(B_{i}^{\prime}\right)
$$

and subsequently prove that $l_{i}^{\prime}\left(B_{i}^{\prime}\right)$ is constant in an interval $\left(B_{i}^{\mathrm{D}}-\gamma, B_{i}^{\mathrm{D}}\right]$ for a sufficiently small $\gamma \in \mathbb{R}^{+}$, i.e.

$$
\underset{B_{i}^{\mathrm{D}}-\gamma<B_{i}^{\prime} \leq B_{i}^{\mathrm{D}}}{\forall B_{i}^{\prime}} \quad l_{i}^{\prime}\left(B_{i}^{\prime}\right)=w l_{i}^{\mathrm{D}} .
$$

To prove that $l_{i}^{\prime}\left(B_{i}^{\prime}\right)$ is left-continuous in $B_{i}^{\mathrm{D}}$, we show that $L_{i}^{\prime}\left(B_{i}^{\mathrm{D}}\right)$ is defined and equal to $W L_{i}^{\mathrm{D}}$, and subsequently show that $l_{i}^{\prime}\left(B_{i}^{\mathrm{D}}\right)=w l_{i}^{\mathrm{D}}$. From Theorem 7 , we know that $L_{i}^{\prime}\left(B_{i}^{\prime}\right)$ exists if Assumption 1 holds. Moreover, considering Theorem 6 and Lemma 5, we conclude that $W L_{i}^{\mathrm{D}}$ and $L_{i}^{\prime}\left(B_{i}^{\mathrm{D}}\right)$ are solutions of the same equation, i.e. $L_{i}^{\prime}\left(B_{i}^{\mathrm{D}}\right)=W L_{i}^{\mathrm{D}}$. As a result, we get

$$
l_{i}^{\prime}\left(B_{i}^{\mathrm{D}}\right)=\left\lceil\frac{L_{i}^{\prime}\left(B_{i}^{\mathrm{D}}\right)}{T_{i}}\right\rceil=\left\lceil\frac{W L_{i}^{\mathrm{D}}}{T_{i}}\right\rceil=w l_{i}^{\mathrm{D}} .
$$

To prove that $l_{i}^{\prime}\left(B_{i}^{\prime}\right)$ is constant in an interval $\left(B_{i}^{\mathrm{D}}-\gamma, B_{i}^{\mathrm{D}}\right]$ for a sufficiently small $\gamma \in \mathbb{R}^{+}$, we use the definition of a limit:

$$
\lim _{x \uparrow X} f(x)=Y \quad \Leftrightarrow \quad \underset{\varepsilon>0}{\forall} \underset{\delta>0}{\exists} \underset{x \in(X-\delta, X)}{\forall}|f(x)-Y|<\varepsilon .
$$


Because $l_{i}^{\prime}\left(B_{i}^{\prime}\right)$ is strictly non-decreasing and defined in $B_{i}^{\mathrm{D}}$, we have

$$
\underset{0 \leq B_{i}^{\prime} \leq B_{i}^{\mathrm{D}}}{\forall} \quad l_{i}^{\prime}\left(B_{i}^{\prime}\right) \leq w l_{i}^{\mathrm{D}}
$$

Let $\varepsilon \in(0,1]$. Now there exists a $\delta \in\left(0, B_{i}^{\mathrm{D}}\right)$ such that $0 \leq w l_{i}^{\mathrm{D}}-l_{i}^{\prime}\left(B_{i}^{\prime}\right)<\varepsilon \leq 1$ for all $B_{i}^{\prime} \in\left(B_{i}^{\mathrm{D}}-\delta, B_{i}^{\mathrm{D}}\right]$, hence $w l_{i}^{\mathrm{D}} \geq l_{i}^{\prime}\left(B_{i}^{\prime}\right)>w l_{i}^{\mathrm{D}}-1$. Because $w l_{i}^{\mathrm{D}}, l_{i}^{\prime}\left(B_{i}^{\prime}\right) \in \mathbb{N}$, this completes the proof.

Note that unlike $W L_{i}^{\mathrm{D}}$, the value for $w l_{i}^{\mathrm{D}}$ can be assumed. Based on Lemma 6 , we conclude that $l_{i}^{\prime}\left(\left(B_{i}^{\mathrm{D}}-\gamma\right)^{+}\right)=w l_{i}^{\mathrm{D}}$ for a sufficiently small $\gamma \in \mathbb{R}^{+}$, and we can therefore exchange the order of the operators in (37), i.e.

$$
W R_{i}^{\mathrm{D}}=\max _{0 \leq k<w l_{i}^{\mathrm{D}} \varepsilon \downarrow 0} \lim _{i k} R_{i}^{\prime}\left(\left(B_{i}^{\mathrm{D}}-\varepsilon\right)^{+}\right) .
$$

Hence, $W R_{i k}^{\mathrm{D}}$ is given by

$$
W R_{i k}^{\mathrm{D}}=\lim _{\varepsilon \downarrow 0} R_{i k}^{\prime}\left(\left(B_{i}^{\mathrm{D}}-\varepsilon\right)^{+}\right) .
$$

Lemma 7 The worst-case response time $W R_{i k}^{\mathrm{D}}$ of job $k$ with $0 \leq k<w l_{i}^{\mathrm{D}}$ of a task $\tau_{i}$ under FPDS and arbitrary phasing is given by

$$
W R_{i k}^{\mathrm{D}}= \begin{cases}W R_{i}^{\mathrm{P}}\left(B_{i}^{\mathrm{D}}+(k+1) C_{i}-F_{i}\right)+F_{i}-k T_{i} & \text { for } i<n, \\ W O_{n}^{\mathrm{P}}\left((k+1) C_{n}-F_{n}\right)+F_{n}-k T_{n} & \text { for } i=n,\end{cases}
$$

where $W R_{i}^{\mathrm{P}}\left(B_{i}^{\mathrm{D}}+(k+1) C_{i}-F_{i}\right)$ and $W O_{i}^{\mathrm{P}}\left(B_{i}^{\mathrm{D}}+(k+1) C_{i}-F_{i}\right)$ are the worstcase response time and the worst-case occupied time under FPPS of a task $\tau_{i}^{\prime}$ with a computation time $C_{i}^{\prime}=B_{i}^{\mathrm{D}}+(k+1) C_{i}-F_{i}$, a period $T_{i}^{\prime}=k T_{i}+D_{i}-F_{i}$ and $a$ deadline $D_{i}^{\prime}=T_{i}^{\prime}$.

Proof Starting from (48), we derive

$$
\begin{aligned}
W R_{i k}^{\mathrm{D}} & =\lim _{\varepsilon \downarrow 0} R_{i k}^{\prime}\left(\left(B_{i}^{\mathrm{D}}-\varepsilon\right)^{+}\right) \\
& =\{(35)\} \lim _{\varepsilon \downarrow 0}\left(b_{i k, m_{i}}^{\prime}\left(\left(B_{i}^{\mathrm{D}}-\varepsilon\right)^{+}\right)+F_{i}-k T_{i}\right) \\
& =\lim _{\varepsilon \downarrow 0} b_{i k, m_{i}}^{\prime}\left(\left(B_{i}^{\mathrm{D}}-\varepsilon\right)^{+}\right)+F_{i}-k T_{i},
\end{aligned}
$$

where $b_{i k, m_{i}}^{\prime}\left(\left(B_{i}^{\mathrm{D}}-\varepsilon\right)^{+}\right)$denotes the relative begin time of the final subjob of job $k$ of task $\tau_{i}$ with $0 \leq k<w l_{i}$ and $\varphi_{j}^{\prime}=0$ for $j \leq i$ as given in (36). Hence, $b_{i k, m_{i}}^{\prime}\left(\left(B_{i}^{\mathrm{D}}-\varepsilon\right)^{+}\right)$is the smallest $x \in \mathbb{R}^{+}$satisfying

$$
x=\left(\left(B_{i}^{\mathrm{D}}-\varepsilon\right)^{+}\right)+(k+1) C_{i}-F_{i}+\sum_{j<i}\left(\left\lfloor\frac{x}{T_{j}}\right\rfloor+1\right) C_{j} .
$$


Now let task set $\mathcal{T}^{\prime}$ be identical to $\mathcal{T}$ except for the characteristics of task $\tau_{i}$, i.e. $\tau_{i}^{\prime}$ has characteristics $C_{i}^{\prime}=\left(B_{i}^{\mathrm{D}}-\varepsilon\right)^{+}+(k+1) C_{i}-F_{i}, T_{i}^{\prime}=k T_{i}+D_{i}-F_{i}$, and $D_{i}^{\prime}=T_{i}^{\prime}$. Hence, task $\tau_{i}^{\prime}$ of $\mathcal{T}^{\prime}$ misses its deadline under FPPS and arbitrary phasing if and only if job $k$ of task $\tau_{i}$ of $\mathcal{T}$ misses its deadline under FPDS, and arbitrary phasing and an amount of blocking $\left(B_{i}^{\mathrm{D}}-\varepsilon\right)^{+}$. Based on Theorem 4, we can now write

$$
b_{i k, m_{i}}^{\prime}\left(\left(B_{i}^{\mathrm{D}}-\varepsilon\right)^{+}\right)=W O_{i}^{\mathrm{P}}\left(\left(B_{i}^{\mathrm{D}}-\varepsilon\right)^{+}+(k+1) C_{i}-F_{i}\right) .
$$

For $i=n$, we substitute $B_{n}^{\mathrm{D}}=0$, and immediately arrive at (49), which proves the lemma for $i=n$.

For $i<n$, we derive

$$
\begin{aligned}
W R_{i k}^{\mathrm{D}} & =\lim _{\varepsilon \downarrow 0} W O_{i}^{\mathrm{P}}\left(\left(B_{i}^{\mathrm{D}}-\varepsilon\right)^{+}+(k+1) C_{i}-F_{i}\right)+F_{i}-k T_{i} \\
& =\{(16)\} W R_{i}^{\mathrm{P}}\left(B_{i}^{\mathrm{D}}+(k+1) C_{i}-F_{i}\right)+F_{i}-k T_{i},
\end{aligned}
$$

which proves the lemma for $i<n$.

Note that because the lowest priority task is the only task that cannot be blocked, i.e. $B_{n}^{\mathrm{D}}=0$, the worst-case response time analysis for FPDS is not uniform for all tasks. Moreover, note that $W R_{i k}^{\mathrm{D}}$ is a supremum (and not a maximum) for all tasks, except for the lowest priority task, i.e. that value cannot be assumed for $i<n$.

Theorem 12 The worst-case response time $W R_{i}^{\mathrm{D}}$ of a task $\tau_{i}$ under FPDS and arbitrary phasing is given by

$$
W R_{i}^{\mathrm{D}}=\max _{0 \leq k<w l_{i}^{\mathrm{D}}} W R_{i k}^{\mathrm{D}}
$$

Proof The theorem follows immediately from (47) and (48), and requires Lemma 7.

\subsection{An iterative procedure}

The next theorem provides an iterative procedure to determine the worst-case response time $W R_{i}^{\mathrm{D}}$ for task $\tau_{i}$ under FPDS and arbitrary phasing. The procedure is stopped when the worst-case response time $W R_{i k}^{\mathrm{D}}$ of job $k$ for task $\tau_{i}$ exceeds the deadline $D_{i}$ or when the level- $i$ active period is over. This latter condition is based on a property of $W L_{i}^{\mathrm{D}}$.

Lemma 8 The worst-case length $W L_{i k}^{\mathrm{D}}$ of a level-( $\left.i, k\right)$ active period under FPDS is the smallest positive $x \in \mathbb{R}^{+}$satisfying the following equation

$$
x=B_{i}^{\mathrm{D}}+(k+1) C_{i}+\sum_{j<i}\left\lceil\frac{x}{T_{j}}\right\rceil C_{j} .
$$

Proof The proof is similar to the proof of Lemma 5. 
Note that because $B_{i}^{\mathrm{D}}$ is a supremum (and not a maximum) for all tasks, except the lowest priority task, $W L_{i k}^{\mathrm{D}}$ is also supremum (and not a maximum) for all tasks, except the lowest priority task, i.e. that value cannot be assumed for $i<n$.

Lemma 9 The worst-case length $W L_{i k}^{\mathrm{D}}$ of a level-( $\left.i, k\right)$ active period under FPDS is given by

$$
W L_{i k}^{\mathrm{D}}=W R_{i}^{\mathrm{P}}\left(B_{i}^{\mathrm{D}}+(k+1) C_{i}\right),
$$

where $W R_{i}^{\mathrm{P}}\left(B_{i}^{\mathrm{D}}+(k+1) C_{i}\right)$ is the worst-case response time under FPPS and arbitrary phasing of a task $\tau_{i}^{\prime}$ with a computation time $C_{i}^{\prime}=B_{i}^{\mathrm{D}}+(k+1) C_{i}$, a period $T_{i}^{\prime}=k T_{i}+D_{i}$ and a deadline $D_{i}^{\prime}=T_{i}^{\prime}$.

Proof The lemma follows from the similarity between (9) and (51). The period and deadline of task $\tau_{i}^{\prime}$ have been chosen to be equal to the deadline of job $k+1$ of task $\tau_{i}$. Hence, when the iterative procedure to determine $W R_{i}^{\mathrm{P}}\left(B_{i}^{\mathrm{D}}+(k+1) C_{i}\right)$ stops because the deadline $D_{i}^{\prime}$ is exceeded, the deadline $d_{i, k+1}$ will be exceeded as well.

Lemma 10 Let $k^{\prime} \in \mathbb{N}$ be the smallest value for which $W R_{i}^{\mathrm{P}}\left(B_{i}^{\mathrm{D}}+\left(k^{\prime}+1\right) C_{i}\right) \leq$ $\left(k^{\prime}+1\right) T_{i}$. The worst-case length $W L_{i}^{\mathrm{D}}$ of a level-i active period is now given by $W R_{i}^{\mathrm{P}}\left(B_{i}^{\mathrm{D}}+\left(k^{\prime}+1\right) C_{i}\right)$.

Proof To prove the lemma, we will prove the following equivalent relation by means of a contradiction argument

$$
\underset{0 \leq k<w l_{i}^{\mathrm{D}}}{\forall} \quad\left(W L_{i k}^{\mathrm{D}} \leq(k+1) T_{i} \quad \Rightarrow \quad k=w l_{i}^{\mathrm{D}}-1\right) .
$$

We only consider $k<w l_{i}^{\mathrm{D}}-1$, because the proof for $k=w l_{i}^{\mathrm{D}}-1$ is similar.

Let $W L_{i, k}^{\mathrm{D}} \leq(k+1) T_{i}$ for $0 \leq k<w l_{i}^{\mathrm{D}}-1$. Using Lemma 9, we derive $W R_{i}^{\mathrm{P}}\left(B_{i}^{\mathrm{D}}+\right.$ $\left.(k+1) C_{i}\right) \leq(k+1) T_{i}$. Hence, task $\tau_{i}^{\prime}$ has a completion at or before $(k+1) T_{i}$, and all higher priority tasks that are released in the interval $\left[0, W R_{i}^{\mathrm{P}}\left(B_{i}^{\mathrm{D}}+(k+1) C_{i}\right)\right)$ have a completion in that interval. Because task $\tau_{i}^{\prime}$ represents the executions of both the blocking lower priority task as well as task $\tau_{i}$, all executions of the corresponding jobs also have a completion in that interval. Hence, the level- $i$ active period that started with an $\varepsilon$-critical instant ends at time $W R_{i}^{\mathrm{P}}\left(B_{i}^{\mathrm{D}}+(k+1) C_{i}\right)$. However, we now have that the length of the level- $i$ active period equals $W L_{i, k}^{\mathrm{D}}$, a value that is strictly smaller than $W L_{i}^{\mathrm{D}}$, which is a contradiction. Therefore, our assumption that $W L_{i, k}^{\mathrm{D}} \leq(k+1) T_{i}$ for $0 \leq k<w l_{i}^{\mathrm{D}}-1$ is wrong, which proves the lemma.

Theorem 13 The worst-case response time $W R_{i}^{\mathrm{D}}$ of a task $\tau_{i}$ can be found by the following iterative procedure under Assumption 1, using (49):

$$
\begin{aligned}
W R_{i}^{(0)} & =W R_{i, 0}^{\mathrm{D}}, \\
W R_{i}^{(l+1)} & =\max \left(W R_{i}^{(l)}, W R_{i, l+1}^{\mathrm{D}}\right), \quad l=0,1, \ldots
\end{aligned}
$$


The procedure is stopped when the worst-case response time $W R_{i k}^{\mathrm{D}}$ of job $k$ of task $\tau_{i}$ exceeds the deadline $D_{i}$ or when the level-i active period is over, i.e. $W R_{i}^{\mathrm{P}}\left(B_{i}^{\mathrm{D}}+\right.$ $\left.(k+1) C_{i}\right) \leq(k+1) T_{i}$.

Proof Lemma 10 states that $W R_{i}^{\mathrm{P}}\left(B_{i}^{\mathrm{D}}+(k+1) C_{i}\right) \leq(k+1) T_{i}$ is a proper termination condition to determine whether or not the level- $i$ active period is over before the release of job $k+1$. Because of Theorem 7, the level- $i$ active periods ends under Assumption 1, and we therefore have to consider at most a finite number $w l_{i}^{\mathrm{D}}$ of jobs of task $\tau_{i}$. As a result, the iterative procedure ends. We observe that the iterative procedure also stops when the deadline $D_{i}$ is exceeded, by the worst-case response time $W R_{i k}^{\mathrm{D}}$ of job $k$ of $\tau_{i}$ i.e. when the task set is not schedulable.

Corollary 7 When Assumption 1 holds, we can derive the schedulability of a set of tasks $\mathcal{T}$ under FPDS and arbitrary phasing by checking the schedulability criterion $W R_{i}^{\mathrm{D}} \leq D_{i}$ using Theorem 13 .

Corollary 8 To check the schedulability criterion $W R_{i}^{\mathrm{D}} \leq D_{i}$ we do not need to determine the length $W L_{i}^{\mathrm{D}}$ of the worst-case level-i active period under FPDS first. Instead, we can simply check whether or not the level-i active period is over after every iteration.

Finally note that:

- $W R_{i, k}^{\mathrm{D}}$ can be used as initial value to calculate $W R_{i}^{\mathrm{P}}\left(B_{i}^{\mathrm{D}}+(k+1) C_{i}\right)$ to determine whether or not the level- $i$ active period is over before the release of job $k+1$.

- $W R_{i}^{\mathrm{P}}\left(B_{i}^{\mathrm{D}}+(k+1) C_{i}\right)$ can be used as initial value to calculate $W R_{i}^{\mathrm{P}}\left(B_{i}^{\mathrm{D}}+\right.$ $\left.(k+2) C_{i}-F_{i}\right)$ to determine $W R_{i, k+1}^{\mathrm{D}}$.

\section{Examples}

In this section, we will illustrate the worst-case response time analysis presented in Sect. 6 to determine the schedulability of tasks and task sets under FPDS and arbitrary phasing of some examples of Sect. 4 using the iterative procedure presented in Theorem 13.

\subsection{Schedulability of task $\tau_{2}$ of $\mathcal{T}_{2}$}

The schedulability of task $\tau_{2}$ of task set $\mathcal{T}_{2}$ is the topic of this section. The characteristics of the tasks of $\mathcal{T}_{2}$ can be found in Table 2 in Sect. 4.2.

To determine the worst-case response time $W R_{2}^{\mathrm{D}}$ for task $\tau_{2}$, we first derive $B_{2}^{\mathrm{D}}=2$ using (19). Next, we determine $W R_{2}^{(0)}$ using Lemma 7, i.e.

$$
W R_{2}^{(0)}=W R_{2,0}^{\mathrm{D}}=W R_{2}^{\mathrm{P}}\left(B_{2}^{\mathrm{D}}+C_{2}-F_{2}\right)+F_{2}=W R_{2}^{\mathrm{P}}(3)+2=5+2=7 .
$$

Because $W R_{2,0}^{\mathrm{D}} \leq D_{2}=7$ and $W R_{2}^{\mathrm{P}}\left(B_{2}^{\mathrm{D}}+C_{2}\right)=W R_{2}^{\mathrm{P}}(5)=9>T_{2}=7$, i.e. the level-2 active period is not over yet, we proceed with the 2 nd job. 
For the 2nd job, we find

$$
W R_{2,1}^{\mathrm{D}}=W R_{2}^{\mathrm{P}}\left(B_{2}^{\mathrm{D}}+2 C_{2}-F_{2}\right)+F_{2}-T_{2}=W R_{2}^{\mathrm{P}}(6)-5=10-5=5,
$$

and therefore $W R_{2}^{(1)}=\max \left(W R_{2}^{(0)}, W R_{2,1}^{\mathrm{D}}\right)=\max (7,5)=7$. Now $W R_{2,1}^{\mathrm{D}}=5 \leq D_{2}$ and $W R_{2}^{\mathrm{P}}\left(B_{2}^{\mathrm{D}}+2 C_{2}\right)=W R_{2}^{\mathrm{P}}(8)=14 \leq 2 T_{2}=14$. Hence, we know that the level-2 active period is over, all jobs of task $\tau_{2}$ meet their deadlines in that period, and the worst-case response time $W R_{2}^{\mathrm{D}}=7$.

\subsection{Schedulability of task $\tau_{2}$ of $\mathcal{T}_{4}$}

We will determine the schedulability of task $\tau_{2}$ of task set $\mathcal{T}_{4}$ in this section. The characteristics of the tasks of $\mathcal{T}_{4}$ can be found in Table 4 in Sect. 4.3.2.

We first determine $W R_{2}^{(0)}$ using Lemma 7, i.e.

$$
W R_{2}^{(0)}=W R_{2,0}^{\mathrm{D}}=W O_{2}^{\mathrm{P}}\left(C_{2}-F_{2}\right)+F_{2}=W O_{2}^{\mathrm{P}}(2)+2.1=4+2.1=6.1 .
$$

Because $W R_{2,0}^{\mathrm{D}} \leq D_{2}=7$ and $W R_{2}^{\mathrm{P}}\left(B_{2}^{\mathrm{D}}+C_{2}\right)=W R_{2}^{\mathrm{P}}(4.1)=8.1>T_{2}=7$, we proceed with the 2 nd job.

For the 2nd job, we find

$$
W R_{2,1}^{\mathrm{D}}=W O_{2}^{\mathrm{P}}\left(2 C_{2}-F_{2}\right)+F_{2}-T_{2}=W O_{2}^{\mathrm{P}}(6.1)-4.9=12.1-4.9=7.2 .
$$

Because $W R_{2,1}^{\mathrm{D}}>D_{2}=7$, we conclude that task $\tau_{2}$ is not schedulable.

\subsection{Schedulability of the task set $\mathcal{T}_{5}$}

In this section, we will determine the schedulability of the task set $\mathcal{T}_{5}$. The characteristics of the tasks of $\mathcal{T}_{5}$ can be found in Table 5 in Sect. 4.3.3.

To determine the worst-case response time $W R_{1}^{\mathrm{D}}$ for task $\tau_{1}$, we first derive $B_{1}^{\mathrm{D}}=3$ using (19). Next, we determine $W R_{2}^{(0)}$ using Lemma 7, i.e.

$$
W R_{1}^{(0)}=W R_{1,0}^{\mathrm{D}}=W R_{1}^{\mathrm{P}}\left(B_{1}^{\mathrm{D}}+C_{1}-F_{1}\right)+F_{1}=3+2=5 .
$$

Now $W R_{1,0}^{\mathrm{D}}=D_{1}$ and $W R_{1}^{\mathrm{D}}\left(B_{1}^{\mathrm{D}}+C_{1}\right)=5=T_{1}$. Hence, we know that the level-1 active period is over, all jobs of task $\tau_{1}$ meet their deadlines, and the worst-case response time $W R_{1}^{\mathrm{D}}=5$.

Next, we determine the worst-case response time $W R_{2}^{\mathrm{D}}$ for task $\tau_{2}$. To this end, we first determine $W R_{2}^{(0)}$ using Lemma 7, i.e.

$$
W R_{2}^{(0)}=W R_{2,0}^{\mathrm{D}}=W O_{2}^{\mathrm{P}}\left(C_{2}-F_{2}\right)+F_{2}=W O_{2}^{\mathrm{P}}(1.2)+3=3.2+3=6.2 .
$$

Because $W R_{2,0}^{\mathrm{D}}<D_{2}=7$ and $W R_{2}^{\mathrm{P}}\left(B_{2}^{\mathrm{D}}+C_{2}\right)=8.2>T_{2}=7$, we proceed with the 2nd job.

For the 2nd job, we find

$$
W R_{2,1}^{\mathrm{D}}=W O_{2}^{\mathrm{P}}\left(2 C_{2}-F_{2}\right)+F_{2}-T_{2}=W O_{2}^{\mathrm{P}}(5.4)-4=9.4-4=5.4,
$$


and therefore $W R_{2}^{(1)}=\max \left(W R_{2}^{(0)}, W R_{2,1}^{\mathrm{D}}\right)=\max (6.2,5.4)=6.2$. Because $W R_{2,1}^{\mathrm{D}}<D_{2}$ and $W R_{2}^{\mathrm{P}}\left(B_{2}^{\mathrm{D}}+2 C_{2}\right)=14.4>2 T_{2}=14$, we proceed with the $3 \mathrm{rd}$ job.

For the 3rd job, we find

$$
W R_{2,2}^{\mathrm{D}}=W O_{2}^{\mathrm{P}}\left(3 C_{2}-F_{2}\right)+F_{2}-2 T_{2}=W O_{2}^{\mathrm{P}}(9.6)-11=17.6-11=6.6,
$$

and therefore $W R_{2}^{(2)}=\max \left(W R_{2}^{(1)}, W R_{2,2}^{\mathrm{D}}\right)=\max (6.2,6.6)=6.6$. Because $W R_{2,2}^{\mathrm{D}}<D_{2}$ and $W R_{2}^{\mathrm{P}}\left(B_{2}^{\mathrm{D}}+3 C_{2}\right)=22.6>3 T_{2}=21$, we proceed with the 4 th job.

For the 4th job, we find

$$
W R_{2,3}^{\mathrm{D}}=W O_{2}^{\mathrm{P}}\left(4 C_{2}-F_{2}\right)+F_{2}-3 T_{2}=W O_{2}^{\mathrm{P}}(13.8)-18=23.8-18=5.8,
$$

and therefore $W R_{2}^{(3)}=\max \left(W R_{2}^{(2)}, W R_{2,3}^{\mathrm{D}}\right)=\max (6.6,5.8)=6.6$. Because $W R_{2,3}^{\mathrm{D}}<D_{2}$ and $W R_{2}^{\mathrm{P}}\left(B_{2}^{\mathrm{D}}+4 C_{2}\right)=28.8>4 T_{2}=28$, we proceed with the 5 th job.

For the 5 th job, we find

$$
W R_{2,4}^{\mathrm{D}}=W O_{2}^{\mathrm{P}}\left(5 C_{2}-F_{2}\right)+F_{2}-4 T_{2}=W O_{2}^{\mathrm{P}}(18)-25=32-25=7,
$$

and therefore $W R_{2}^{(4)}=\max \left(W R_{2}^{(3)}, W R_{2,4}^{\mathrm{D}}\right)=\max (6.6,7)=7$. Now $W R_{2,4}^{\mathrm{D}}=D_{2}$ and $W R_{2}^{\mathrm{P}}\left(B_{2}^{\mathrm{D}}+5 C_{2}\right)=35=5 T_{2}$. Hence we know that the level-2 active period is over, all jobs of task $\tau_{2}$ meet their deadlines in that period, and the worst-case response time $W R_{2}^{\mathrm{D}}=7$.

Because $W R_{i}^{\mathrm{D}} \leq D_{i}$ for all $i \leq n$, we conclude that $\mathcal{T}_{5}$ is schedulable under FPDS and arbitrary phasing when deadlines are equal to periods.

\section{Discussion}

This section presents a theorem for the worst-case response time of the highest priority task, compares the notion of level- $i$ active period with similar notions in the literature, presents pessimistic variants for the worst-case response time analysis of tasks, illustrates the revised analysis for an advanced model for FPDS, and shows that our analysis is sustainable.

\subsection{Worst-case response time of highest priority task}

In Sect. 4.4, we concluded that the optimism in the existing analysis does not occur for the highest priority task. The next theorem provides a formal basis for that conclusion, by stating that the worst-case response time of the highest priority task $\tau_{1}$ can be found by only considering the first job of $\tau_{1}$ in a level-1 active period started at an $\varepsilon$-critical instant. Intuitively, a later job $k$ of $\tau_{1}$ with $k>1$ in a level-i active period can only have a longer response time than the first job when it is deferred by its previous job for a longer time than the time the first job is blocked by a task with a lower priority than $\tau_{1}$. This would require that the utilization $U_{1}^{\tau}$ of $\tau_{1}$ is larger than 1 , however.

First, we prove the following lemma. 
Lemma 11 The first job of task $\tau_{1}$ in a level-1 active period has the largest response time of all jobs of $\tau_{1}$ in that period.

Proof The highest priority task $\tau_{1}$ experiences blocking of at most one subjob of a lower priority task. If the first job of $\tau_{1}$ in a level-1 active period is blocked by an amount $B$, its response time $R_{1,0}^{\prime}(B)$ becomes

$$
R_{1,0}^{\prime}(B)=B+C_{1} .
$$

Now, assume the level-1 active period contains $l_{1}>1$ jobs of task $\tau_{1}$. The response time $R_{1, k}^{\prime}(B)$ of job $k$, with $0 \leq k<l_{1}$, becomes

$$
\begin{aligned}
R_{1, k}^{\prime}(B) & =B+(k+1) C_{1}-k T_{1} \\
& =B+C_{1}+k\left(C_{1}-T_{1}\right) \\
& =B+C_{1}+k\left(U_{1}-1\right) T_{1} .
\end{aligned}
$$

Because we assume $U \leq 1$, we must have $U_{1}^{\tau}<1$ when task $\tau_{1}$ is blocked by a lower priority task. Hence, we find

$$
R_{1, k}^{\prime}(B)<B+C_{1}=R_{1,0}^{\prime}(B)
$$

which proves the lemma.

Theorem 14 The worst-case response time $W R_{1}^{\mathrm{D}}$ of the highest priority task $\tau_{1}$ under FPDS is equal to

$$
W R_{1}^{\mathrm{D}}=B_{1}^{\mathrm{D}}+C_{1}
$$

Proof From equation $R_{1,0}^{\prime}(B)=B+C_{1}$, we conclude that $R_{1,0}^{\prime}(B)$ is a strictly increasing function of $B$. Hence, we derive

$$
W R_{1}^{\mathrm{D}}=\sup _{B} R_{1,0}^{\prime}(B)=\lim _{B \uparrow B_{1}^{\mathrm{D}}}\left(B+C_{1}\right)=B_{1}^{\mathrm{D}}+C_{1},
$$

which proves the theorem.

\subsection{A comparison with existing notions}

We will now compare our notion of level- $i$ active period with similar notions in the literature.

\subsubsection{Level-i busy period in Lehoczky (1990)}

The notion of level-i busy period originates from Lehoczky (1990), where it has been introduced as an expedient to determine the worst-case response times of tasks for deadlines larger than periods under FPPS and arbitrary phasing. The level- $i$ busy period is defined as follows. 
Definition 11 A level- $i$ busy period is a time interval $[a, b]$ within which jobs of priority $i$ or higher are processed throughout $[a, b]$ but no jobs of level $i$ or higher are processed in $(a-\varepsilon, a)$ or $(b, b+\varepsilon)$ for sufficiently small $\varepsilon>0$.

Figure 9 also shows the level-1 busy periods and level-2 busy periods for $\mathcal{T}_{1}$. The level-1 busy periods in this figure only differ from the level- 1 active periods by the inclusion of the end-points of the intervals by the former. The difference between level-2 busy periods and level-2 active periods is more significant, however. Whereas the interval $[0,12)$ is constituted by four level- 2 active periods, i.e. $[0,5),[5,7)$, $[7,10)$, and $[10,12)$, the interval is contained in a single level-2 busy period $[0,12]$. Stated in other words, the level-2 busy period unifies four adjacent level-2 active periods. Similarly, the interval $[20,27)$ is constituted by two level- 2 active periods, i.e. $[20,25)$ and $[25,27)$, and the interval is contained in a single level-2 busy period $[20,27]$.

Figure 10 shows the level- 1 busy periods and level- 2 busy periods for $\mathcal{T}_{1}$. From this figure, we see that the level-2 busy period never ends for $U=1$, as also becomes immediately clear from Definition 11 . Conversely, the level-2 active period that started at time $t=0$ ends at time $t=35$; see also Assumption 1 and Theorem 7. We observe that the definition of level-i busy period is included in Klein et al. (1993) (on page D-4, referring to Lehoczky (1990)), and the notion is used in Technique 5 "Calculating Response Time with Arbitrary Deadlines and Blocking." As shown above, the busy period never ends for $U=1$. Notably, in Klein et al. (1993, pp. 4-35) it is only mentioned that we must be sure that the [...] utilization [...] is not greater than one. In Step 6 "Decide if the busy period is over" the notion is used to determine whether or not the iterative procedure can be stopped. Notably, that decision is not based on the end of the busy period, but on the end of the level- $i$ active period, i.e. when the (worst-case) response time $W R_{i k}^{\mathrm{P}}$ of job $k$ of task $\tau_{i}$ is less than or equal to $T_{i}$; see also Theorem 13.

There is another striking difference between the level- $i$ active period and the level- $i$ busy period. A level- $i$ active period may start when a task with a lower priority is still being processed, as illustrated by the level-1 active period that starts at time $t=5$ in Fig. 10. The corresponding level-1 busy period does not start at time $t=5$, but at time $t=6.2$ instead.

The fundamental difference between both notions can be traced back to their definitions; a busy period is based on processing of jobs, whereas an active period is based on (pending) load or active jobs.

\subsection{2 $\tau_{i}$-Busy period in González Harbour et al. (1991)}

In González Harbour et al. (1991), the notion of busy period is slightly modified to accommodate the fact that a task $\tau_{i}$ may be composed of distinct subtasks, each of which may have its own timing requirements and fixed priority. In the following definition, $\rho_{i}$ denotes the minimum priority of the subtasks of task $\tau_{i}$.

Definition 12 A $\tau_{i}$-idle instant is any time $t$ such that all work of priority $\rho_{i}$ or higher started before $t$ and all $\tau_{i}$ jobs also started before $t$ have completed at or before $t$. 
Definition 13 A $\tau_{i}$-busy period is an interval of time $[A, B]$ such that both $A$ and $B$ are $\tau_{i}$-idle instants and there is no time $t \in(A, B)$ such that $t$ is a $\tau_{i}$-idle instant.

This notion of $\tau_{i}$-busy period is similar to our level- $i$ active period, with as main difference that a $\tau_{i}$-busy period is a closed interval rather than a right semi-open interval. Although this difference may be viewed as philosophical, we prefer the usage of a right semi-open interval, which we will motivate by means of Fig. 10. Given Definition 12 and 13, time $t=35$ belongs to two $\tau_{2}$-busy periods, i.e. [0,35] and [35, 70]. Moreover, time $t=35$ is also a $\tau_{2}$-idle instant. Hence, $\tau_{i}$-busy periods can overlap, and when they overlap, the overlap is termed a $\tau_{i}$-idle instant. This is considered to be counter-intuitive.

\subsubsection{Level-i busy period in George et al. (1996)}

After a brief recapitulation of the notion of level- $i$ busy period of Lehoczky (1990) for FPPS, an informal description of a level- $i$ busy period for FPNS under discrete scheduling (Baruah et al. 1990b) is given in George et al. (1996, Appendix A.2). Note that for discrete scheduling, all task parameters are integers, i.e. $T_{i}, C_{i}, D_{i} \in \mathbb{Z}^{+}$and $\varphi_{i} \in \mathbb{Z}^{+} \cup\{0\}$, and preemptions are restricted to integer time points.

Unfortunately, there is an inconsistency in George et al. (1996). In Appendix A.2, the following definition is given.

Definition 14 A level-i busy period is a processor busy period in which only instances of tasks with a priority greater than or equal to that of $\tau_{i}$ execute.

Accordingly, the interval of time that a lower priority task blocks task $\tau_{i}$ and its higher priority tasks is not included in the level- $i$ busy period in both the text of the proof of Lemma 6 in Sect. 4.3.1 and Fig. 6, which is used to illustrate that proof. Conversely, that interval is included in the equation to determine the length of the level- $i$ busy period for the non-preemptive case, as described in George et al. (1996, Appendix A.2).

Note that George et al. (1996) does not reproduce the definition of Lehoczky (1990) (see Definition 11 above), but presents a new definition. Surprisingly, the differences between these definitions are not discussed. As an example, a (synchronous processor) busy period in George et al. (1996) is described as a right semi-open interval on page 6, whereas the level- $i$ busy period in Lehoczky (1990) is a closed interval.

The notion of level- $i$ busy period for FPNS in George et al. (1996) is similar to our notion of level- $i$ active period under the assumption that the equation to determine the length of a level- $i$ busy period for the non-preemptive case properly reflects the intention of the authors.

\subsubsection{Level- $\pi_{i}$ busy interval in Liu (2000)}

In Liu (2000), an analysis method is described to determine the schedulability of tasks under FPPS whose relative deadlines are larger than their respective periods, using 
the term level- $\pi_{i}$ busy interval. A level- $\pi_{i}$ busy interval is defined as a left semi-open interval $\left(t_{0}, t\right]$, i.e. the partitioning of the timeline in Liu (2000) differs from ours. Given the description in Liu (2000), our definition of level- $i$ active period can be viewed as a slightly modified definition of level- $\pi_{i}$ busy interval to accommodate our scheduling model for FPDS.

\subsection{Pessimistic variants}

Given (49) in Lemma 7, we observe that the worst-case response time analysis is not uniform for all tasks. The analysis can be made uniform at the cost of potentially introducing pessimism. This section presents two lemmas with pessimistic variants for the worst-case response time analysis, one based on worst-case occupied times and one based on worst-case response times. For both variants, the iterative procedure presented in Theorem 13 can be used, i.e. only the equations for $W R_{i k}^{\mathrm{D}}$ change, not the iterative procedure. We conclude this section with a retrospect on the analysis for FPDS.

\subsubsection{A uniform analysis based on $\mathrm{WO}^{\mathrm{P}}$}

Lemma 12 A pessimistic worst-case response time $\widehat{W R}_{i k}^{\mathrm{D}}$ of job $k$ with $0 \leq k<w l_{i}^{\mathrm{D}}$ of a task $\tau_{i}$ under FPDS and arbitrary phasing is given by

$$
\widehat{W R}_{i k}^{\mathrm{D}}=W O_{i}^{\mathrm{P}}\left(B_{i}^{\mathrm{D}}+(k+1) C_{i}-F_{i}\right)+F_{i}-k T_{i},
$$

where $W O_{i}^{\mathrm{P}}\left(B_{i}^{\mathrm{D}}+(k+1) C_{i}-F_{i}\right)$ is the worst-case occupied time under FPPS of a task $\tau_{i}^{\prime}$ with a computation time $C_{i}^{\prime}=B_{i}^{\mathrm{D}}+(k+1) C_{i}-F_{i}$, a period $T_{i}^{\prime}=k T_{i}+$ $D_{i}-F_{i}$, and a deadline $D_{i}^{\prime}=T_{i}^{\prime}$.

Proof By definition, $W R_{i}^{\mathrm{P}}(C) \leq W O_{i}^{\mathrm{P}}(C)$, hence $W R_{i k}^{\mathrm{D}} \leq \widehat{W R}_{i k}^{\mathrm{D}}$. Because $W R_{1}^{\mathrm{P}}(C)=$ $W O_{1}^{\mathrm{P}}(C), \widehat{W R}_{i k}^{\mathrm{D}}$ is potentially pessimistic for $1<i<n$.

The pessimism is illustrated by the set $\mathcal{T}_{2}$ consisting of three tasks with characteristics as described in Table 2 in Sect. 4.2. For the worst-case response time $\widehat{W R}_{2,0}^{\mathrm{D}}$ of the first job of task $\tau_{2}$ we find

$$
\begin{aligned}
\widehat{W R}_{2,0}^{\mathrm{D}} & =W O_{2}^{\mathrm{P}}\left(B_{2}^{\mathrm{D}}+C_{2}-F_{2}\right)+F_{2} \\
& =W O_{2}^{\mathrm{P}}(2+3-2)+2 \\
& =W O_{2}^{\mathrm{P}}(3)+2=7+2=9 .
\end{aligned}
$$

Because $\widehat{W R}_{2,0}^{\mathrm{D}}>D_{2}, \mathcal{T}_{2}$ is considered unschedulable under FPDS based on Lemma 12. Conversely, application of Lemma 7 yields a value $W R_{2}^{\mathrm{D}}=7 \leq D_{2}$.

We observe that $\widehat{W R}_{2,0}^{\mathrm{D}}$ is equal to $\widetilde{W R}_{2}^{\mathrm{D}}$ as determined in Sect. 4.2 by means of the existing analysis as presented in Burns (1994) and Burns and Wellings (1997). This equality is not a coincidence, for the following two reasons. Firstly, remember 
that because the characteristics of the tasks of $\mathcal{T}_{2}$ are integral multiples of a value $\delta=1$ and $\Delta=0.2 \leq \delta$, the value for $\widetilde{W R}_{2}^{\mathrm{D}}$ does not change when $\Delta$ is reduced to an arbitrary small positive value, i.e.

$$
\widetilde{W R}_{2}^{\mathrm{D}}=\lim _{\Delta \downarrow 0}\left(W R_{2}^{\mathrm{P}}\left(B_{2}^{\mathrm{D}}+C_{2}-\left(F_{2}-\Delta\right)\right)+\left(F_{2}-\Delta\right)\right) .
$$

Secondly, we can make the following derivation using (12)

$$
\begin{gathered}
\lim _{\Delta \downarrow 0}\left(W R_{2}^{\mathrm{P}}\left(B_{2}^{\mathrm{D}}+C_{2}-\left(F_{2}-\Delta\right)\right)+\left(F_{2}-\Delta\right)\right) \\
\quad=\lim _{\Delta \downarrow 0}\left(W R_{2}^{\mathrm{P}}\left(B_{2}^{\mathrm{D}}+C_{2}-\left(F_{2}-\Delta\right)\right)\right)+F_{2} \\
\quad=W O_{2}^{\mathrm{P}}\left(B_{2}^{\mathrm{D}}+C_{2}-F_{2}\right)+F_{2}=\widehat{W R}_{2,0}^{\mathrm{D}} \quad
\end{gathered}
$$

These two results show that $\widehat{W R}_{2,0}^{\mathrm{D}}=\widetilde{W R}_{2}^{\mathrm{D}}$ for $\mathcal{T}_{2}$.

\subsubsection{A uniform analysis based on $W R^{\mathrm{P}}$}

We will give another pessimistic approach that is uniform for all tasks, which assumes a small positive value $\Delta$ and is based on $W R^{\mathrm{P}}$.

Lemma 13 A pessimistic worst-case response time $\widehat{\widehat{W R}}_{i k}^{\mathrm{D}}$ of job $k$ with $0 \leq k<w l_{i}^{\mathrm{D}}$ of a task $\tau_{i}$ under FPDS and arbitrary phasing is given by

$$
\widehat{\widehat{W R}}_{i k}^{\mathrm{D}}=W R_{i}^{\mathrm{P}}\left(B_{i}^{\mathrm{D}}+(k+1) C_{i}-\left(F_{i}-\Delta\right)\right)+\left(F_{i}-\Delta\right)-k T_{i}
$$

where:

(i) $W R_{i}^{\mathrm{P}}\left(B_{i}^{\mathrm{D}}+(k+1) C_{i}-\left(F_{i}-\Delta\right)\right)$ is the worst-case response time under FPPS of a task $\tau_{i}^{\prime}$ with a computation time $C_{i}^{\prime}=B_{i}^{\mathrm{D}}+(k+1) C_{i}-\left(F_{i}-\Delta\right)$, a period $T_{i}^{\prime}=k T_{i}+D_{i}-\left(F_{i}-\Delta\right)$, and a deadline $D_{i}^{\prime}=T_{i}^{\prime}$.

(ii) $\Delta$ is a sufficiently small positive number.

Proof Because $W R_{1}^{\mathrm{P}}(C)=W O_{1}^{\mathrm{P}}(C)=C, \widehat{W W R}_{1,0}^{\mathrm{D}}=\widehat{W R}_{1,0}^{\mathrm{D}}=W R_{1}^{\mathrm{D}}$. Hence, this approach is not pessimistic for $i=1$. We will now prove that $W R_{i}^{\mathrm{P}}(C+\Delta)-\Delta \geq$ $W O_{i}^{\mathrm{P}}(C)$ for $1<i \leq n$. The potential additional pessimism introduced by (57) now immediately follows from Lemma 12, i.e. $\widehat{\widehat{W R}}_{i k}^{\mathrm{D}} \geq \widehat{W R}_{i k}^{\mathrm{D}}$.

By definition, task $\tau_{i}$ can start executing an additional amount of computation time $\Delta$ after having executed an amount $C$ at time $W O_{i}^{\mathrm{P}}(C)$. Because execution of that additional computation time $\Delta$ takes at least an amount of time $\Delta$, we immediately get $W R_{i}^{\mathrm{P}}(C+\Delta) \geq W O_{i}^{\mathrm{P}}(C)+\Delta$, which proves the theorem.

Based on (12), we first conclude that both lemmas are similar for an arbitrary small positive value of $\Delta$, i.e. $\lim _{\Delta \downarrow 0} \widehat{\widehat{W R}}_{i k}^{\mathrm{D}}=\widehat{W R}_{i k}^{\mathrm{D}}$. The additional pessimism potentially introduced by Lemma 13 is illustrated by the set $\mathcal{T}_{7}$ consisting of three tasks with 
Table 7 Task characteristics of $\mathcal{T}_{7}$

\begin{tabular}{lcc}
\hline & $T_{i}=D_{i}$ & $C_{i}$ \\
\hline$\tau_{1}$ & 6.5 & 3 \\
$\tau_{2}$ & 9 & 3 \\
$\tau_{3}$ & 30 & 3 \\
\hline
\end{tabular}

characteristics as described in Table 7. For this example, the task characteristics are integral multiples of $\delta=0.5$. For $\Delta=0.6>\delta$, we find $\widehat{\widehat{W R}}_{2,0}^{\mathrm{D}}=12$, which is larger than $\tau_{2}$ 's deadline. Conversely, the worst-case response time $\widehat{W R}_{2}^{\mathrm{D}}$ of task $\tau_{2}$ determined by means of Theorem 13 using Lemma 12 yields $\widehat{W R}_{2}^{\mathrm{D}}=W R_{2}^{\mathrm{D}}=9 \leq D_{2}$. For $\Delta=0.4<\delta$, we find $\widehat{\widehat{W R}}_{2,0}^{\mathrm{D}}=9$. For this value of $\Delta, \widehat{\widehat{W R}}_{2,0}^{\mathrm{D}}=\widehat{W R}_{2}^{\mathrm{D}}=W R_{2}^{\mathrm{D}}=$ $9 \leq D_{2}$, and reducing the value of $\Delta$ will not change the value found for $\widehat{W W R}_{2,0} \mathrm{D}$.

The next lemma provides a sufficient condition to guarantee that Lemma 13 introduces no additional pessimism compared to Lemma 12.

Lemma 14 If the greatest common divisor $\left(\mathrm{gcd}^{\mathbb{R}^{+}}\right)$of the periods and computation times of the tasks exists, and is equal to $\delta$, then $\Delta<\delta$ is a sufficient condition to guarantee that Lemma 13 introduces no additional pessimism compared to Lemma 12.

Proof To prove the lemma, it suffices to prove

$$
\Delta<\delta \Rightarrow W R_{i}^{\mathrm{P}}\left(B_{i}^{\mathrm{D}}+(k+1) C_{i}-\left(F_{i}-\Delta\right)\right)-\Delta=W O_{i}^{\mathrm{P}}\left(B_{i}^{\mathrm{D}}+(k+1) C_{i}-F_{i}\right) .
$$

From Theorem 2, we derive that $W R_{i}^{\mathrm{P}}\left(B_{i}^{\mathrm{D}}+(k+1) C_{i}-\left(F_{i}-\Delta\right)\right)$ is given by the smallest $x \in \mathbb{R}^{+}$that satisfies the following equation, provided that $x$ is at most $k T_{i}+$ $D_{i}-\left(F_{i}-\Delta\right)$,

$$
x=B_{i}^{\mathrm{D}}+(k+1) C_{i}-\left(F_{i}-\Delta\right)+\sum_{j<i}\left\lceil\frac{x}{T_{j}}\right\rceil C_{j} .
$$

By substituting $x=x^{\prime}+\Delta$, we get

$$
x^{\prime}=B_{i}^{\mathrm{D}}+(k+1) C_{i}-F_{i}+\sum_{j<i}\left\lceil\frac{x^{\prime}+\Delta}{T_{j}}\right\rceil C_{j} .
$$

When the greatest common divisor $\left(\mathrm{gcd}^{\mathbb{R}^{+}}\right)$of the periods and computation times of the tasks exists and is equal to $\delta$, all task parameters are integral multiples of $\delta$ (by definition), and $x^{\prime}$ will also be an integral multiple of $\delta$. Let $x^{\prime}=n_{x^{\prime}} \cdot \delta$ and $T_{j}=n_{T_{j}} \cdot \delta$ for an arbitrary $j<i$, where $n_{x^{\prime}}, n_{T_{j}} \in \mathbb{N}^{+}$. Now we get

$$
\left\lceil\frac{x^{\prime}+\Delta}{T_{j}}\right\rceil=\left\lceil\frac{n_{x^{\prime}}+\frac{\Delta}{\delta}}{n_{T_{j}}}\right\rceil .
$$


Based on Lemma 20, we conclude

$$
0<\frac{\Delta}{\delta}<1 \Rightarrow\left\lceil\frac{n_{x^{\prime}}+\frac{\Delta}{\delta}}{n_{T_{j}}}\right\rceil=\left\lfloor\frac{n_{x^{\prime}}}{n_{T_{j}}}\right\rfloor+1 .
$$

Hence, if the $\operatorname{gcd}^{\mathbb{R}^{+}}$exists and is equal to $\delta>\Delta$, the smallest $x^{\prime} \in \mathbb{R}^{+}$satisfying the recursive equation given above is a solution for both $W R_{i}^{\mathrm{P}}\left(B_{i}^{\mathrm{D}}+(k+1) C_{i}-\right.$ $\left.\left(F_{i}-\Delta\right)\right)-\Delta$ and $W O_{i}^{\mathrm{P}}\left(B_{i}^{\mathrm{D}}+(k+1) C_{i}-F_{i}\right)$, which proves the lemma.

We finally observe that the analysis presented in Lemma 13 is similar to the revised schedulability analysis for CAN presented in Davis et al. (2007). The latter analysis is an evolutionary improvement of the analysis given by Tindell et al. (1994, 1995), and Tindell and Burns (1994). A fixed value for $\Delta$ is used in Davis et al. (2007), corresponding to the transmission time for a single bit on CAN.

\subsubsection{A retrospect}

Using our notation, the worst-case response time of a task $\tau_{i}$ under FPDS, arbitrary phasing, and deadlines less than or equal to periods, as described in Liu (2000) can be given by $W R_{i}^{\mathrm{P}}\left(B_{i}^{\mathrm{D}}+C_{i}\right)$. As observed in Burns et al. (1993), this analysis is pessimistic, because a task $\tau_{i}$ cannot be preempted while executing its last subjob, i.e. $F_{i}$. The original improvement of the worst-case response time of a task $\tau_{i}$ under FPDS as presented in Burns et al. (1993) was not based on $B_{i}^{\mathrm{D}}$ as given in (19), but on the maximum length of deferred preemption. We interpret this latter phrase as a blocking time $\widehat{B}$ given by ${ }^{5}$

$$
\widehat{\widehat{B}}=\max _{2 \leq j \leq n} \max _{1 \leq k \leq m_{j}} C_{j, k} .
$$

Though pessimistic, this original improvement is correct, i.e. not optimistic. The problem with the analysis in Burns (1994), Burns and Wellings (1997) is caused by the fact that the non-preemptive behavior of the final subjob of task $\tau_{i}$ itself is not taken into account for $i>1$, as illustrated by Fig. 7 in Sect. 4.3 .3 for task $\tau_{2}$. As described in Davis et al. (2007) in the context of schedulability analysis for CAN, this problem can therefore be resolved at the cost of potentially introducing additional pessimism by using $\widehat{B}_{i}^{\mathrm{D}}$, which is given by

$$
\widehat{B}_{i}^{\mathrm{D}}=\max \left(B_{i}^{\mathrm{D}}, F_{i}\right) .
$$

Conversely, the problem with the analysis in Burns (1994), Burns and Wellings (1997) does not occur when $\widehat{B}_{i}^{\mathrm{D}}=B_{i}^{\mathrm{D}}$, i.e. when $B_{i}^{\mathrm{D}} \geq F_{i}$.

\subsection{An advanced model for FPDS}

The model for FPDS described in Sect. 2.2 assumes that each job of a task $\tau_{i}$ consists of a sequence of $m_{i}$ non-preemptable subjobs. In this section, we will illustrate by means of an example how our analytical results can be applied in a context where

${ }^{5}$ From Lemma 11, we conclude that we may ignore the highest priority task in (58). 
Fig. 11 An example of a DAG of subjobs, representing the flow graph of task $\tau_{i}$

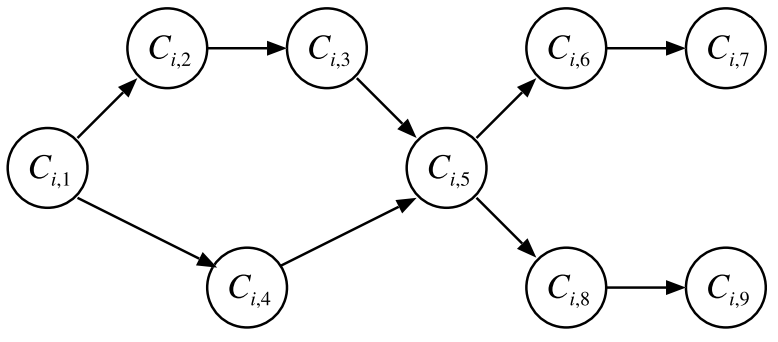

a task $\tau_{i}$ consists of a (rooted and connected) directed acyclic graph (DAG) of $m_{i}$ non-preemptable subjobs.

Consider Fig. 11, with a DAG of subjobs representing the flow graph of task $\tau_{i}$. The nodes of this graph represent the subjobs and the edges represent the successor relationships of subjobs. The graph has a single root node, with a computation time of $C_{i, 1}$, and two leaf nodes, with computation times $C_{i, 7}$ and $C_{i, 9}$, respectively. During the execution of a job, a single path from the root node to a leaf is traversed. Hence, a job will either execute the subjobs with computation times $C_{i, 2}$ and $C_{i, 3}$ or the subjob with computation time $C_{i, 4}$. Similarly, a job will either execute $C_{i, 6}$ and $C_{i, 7}$ or $C_{i, 8}$ and $C_{i, 9}$. The structure of task $\tau_{i}$ plays a role during the analysis of the task itself, and for a lower priority task. The analysis of tasks with a higher priority than $\tau_{i}$ is similar to the case where a job consists of a sequence of subjobs. For the analysis of a task with a lower priority than $\tau_{i}$, we need to determine the longest computation time $C_{i}$ of $\tau_{i}$ for all possible paths through the graph. For our example, this is equal to

$$
C_{i}=C_{i, 1}+\max \left(C_{i, 2}+C_{i, 3}, C_{i, 4}\right)+C_{i, 5}+\max \left(C_{i, 6}+C_{i, 7}, C_{i, 8}+C_{i, 9}\right) .
$$

For the analysis of task $\tau_{i}$ itself, every leaf node of the DAG gives rise to a case that needs to be examined individually. For our example, we therefore get two cases, a first case for the leaf node $C_{i, 7}$, i.e.

$$
\begin{aligned}
& C_{i}^{\prime}=C_{i, 1}+\max \left(C_{i, 2}+C_{i, 3}, C_{i, 4}\right)+C_{i, 5}+C_{i, 6}+C_{i, 7}, \\
& F_{i}^{\prime}=C_{i, 7},
\end{aligned}
$$

and a second case for the leaf node $C_{i, 9}$, i.e.

$$
\begin{aligned}
& C_{i}^{\prime \prime}=C_{i, 1}+\max \left(C_{i, 2}+C_{i, 3}, C_{i, 4}\right)+C_{i, 5}+C_{i, 8}+C_{i, 9}, \\
& F_{i}^{\prime \prime}=C_{i, 9} .
\end{aligned}
$$

The worst-case response time $W R_{i}^{\mathrm{D}}$ of task $\tau_{i}$ is the maximum of the worst-case response times of these two cases. Note that if $C_{i}^{\prime}-F_{i}^{\prime} \geq C_{i}^{\prime \prime}-F_{i}^{\prime \prime}$ and $F_{i}^{\prime} \geq F_{i}^{\prime \prime}$, then it suffices to consider the first case only. Similarly, if $C_{i}^{\prime \prime}-F_{i}^{\prime \prime} \geq C_{i}^{\prime}-F_{i}^{\prime}$ and $F_{i}^{\prime \prime} \geq F_{i}^{\prime}$, then it suffices to consider only the second case. As an alternative, we can also take a pessimistic approach, and determine $W R_{i}^{\mathrm{D}}$ based on

$$
\begin{aligned}
\widehat{C}_{i} & =\max \left(C_{i}^{\prime}-F_{i}^{\prime}, C_{i}^{\prime \prime}-F_{i}^{\prime \prime}\right)+\max \left(F_{i}^{\prime}, F_{i}^{\prime \prime}\right), \\
\widehat{F}_{i} & =\max \left(F_{i}^{\prime}, F_{i}^{\prime \prime}\right) .
\end{aligned}
$$


Table 8 Task characteristics of $\mathcal{T}_{8}$

\begin{tabular}{llr}
\hline & $T_{i}=D_{i}$ & $C_{i}$ \\
\hline$\tau_{1}$ & 16 & 2 \\
$\tau_{2}$ & 24 & 15 \\
$\tau_{3}$ & 36 & 3 \\
\hline
\end{tabular}

We will now illustrate the analysis for $\tau_{i}$ with a numerical example. Consider the set $\mathcal{T}_{8}$ in Table 8. Assume a structure of each job of $\tau_{2}$ as illustrated in Fig. 11, and let the computation times of the subjobs of task $\tau_{2}$ be given by $C_{2,1}=1, C_{2,2}=3$, $C_{2,3}=4, C_{2,4}=6, C_{2,5}=1, C_{2,6}=3, C_{2,7}=2, C_{2,8}=1, C_{2,9}=5$. We now find $C_{2}^{\prime}=1+\max (3+4,6)+1+3+2=14, F_{2}^{\prime}=2, C_{2}^{\prime \prime}=1+\max (3+4,6)+1+$ $1+5=15$, and $F_{2}^{\prime \prime}=5$. Because $C_{2}^{\prime}-F_{2}^{\prime}=12>C_{2}^{\prime \prime}-F_{2}^{\prime \prime}=10$ and $F_{2}^{\prime}=2<$ $F_{2}^{\prime \prime}=5$, we have to determine the worst-case response times for both cases. Using the analysis presented in Sect. 6, we find 21 for the first case and 20 for the second case. The worst-case response time of $\tau_{2}$ is therefore assumed for the first case, i.e. $W R_{2}^{\mathrm{D}}=21$. For the pessimistic approach, we find $\widehat{C}_{2}=\max (12,10)+$ $\max (2,5)=17, \widehat{F}_{2}=5$, and derive a worst-case response time for task $\tau_{2}$ equal to 24 .

\subsection{A note on sustainability}

This section briefly discusses the impact of assuming worst-case rather than fixed computation times and minimum inter-arrival times (or worst-case periods) rather than fixed periods. Stated differently, we consider the sustainability (Baruah and Burns 2006) of our schedulability analysis for FPDS.

As described in Baruah and Burns (2006), a schedulability test is sustainable if any task system deemed schedulable by the test remains so if it behaves 'better' than mandated by its system specifications, i.e. sustainability requires that schedulability be preserved in situations in which it should be 'easier' to ensure schedulability. The concept of sustainability is defined as

Definition 15 (From Baruah and Burns 2006) A schedulability test for a scheduling policy is sustainable if any system deemed schedulable by the schedulability test remains schedulable when the parameters of one or more individual job[s] are changed in any, some, or all of the following ways: (i) decreased execution requirements; (ii) later arrival times; (iii) smaller jitter; and (iv) larger relative deadlines.

That paper also proves that response time analysis for FPPS is sustainable.

For FPDS, we have to adapt Definition 15 to our model, i.e. Definition 15 is based on a model that only considers jobs of tasks and does not explicitly consider subjobs of tasks.

Definition 16 A schedulability test for our real-time scheduling model for FPDS is sustainable if any system deemed schedulable by the test remains schedulable when the parameters are changed in any, some, or all of the following ways: (i) decreased 
execution requirements of subjobs; (ii) later arrival times of jobs; (iii) larger relative deadlines of jobs.

With such an adaptation, the schedulability analysis for our model for FPDS is sustainable, as expressed by the following theorem, for which we merely provide a sketch of a proof.

Theorem 15 Based on Definition 16, the schedulability analysis for our model of FPDS as expressed by the schedulability test (3) Theorem 12 and Lemmas 7, 12, and 13 is sustainable.

Sketch of proof Sustainability of our schedulability analysis for FPDS immediately follows from (3) and the fact that:

- the maximum number $w l_{i}^{\mathrm{D}}$ of jobs of task $\tau_{i}$ in a level- $i$ active period, and

- the (pessimistic) worst-case response times $W R_{i k}^{\mathrm{D}}$ in (49), $\widehat{W R}_{i k}^{\mathrm{D}}$ in (56), and $\widehat{\widehat{W R}}_{i k}^{\mathrm{D}}$ in (57) are strictly non-increasing for decreasing computation times of subjobs and increasing periods of tasks.

Based on Theorem 15, we conclude that we can replace computation times by worst-case computation times and periods by minimum inter-arrival times (or worstcase periods) in our real-time scheduling models in Sect. 2.

To illustrate the significance of our adaptation let's consider an example showing that Definition 15 is not sufficient for our model. In particular, we show that a schedulable task system under FPDS becomes unschedulable when the computation time $C$ of a task remains the same, but its distribution to the task its subjobs changes. As an example, the task characteristics of $\mathcal{T}_{9}$ in Table 9 only differ of those of $\mathcal{T}_{5}$ in Table 5 in Sect. 4.3.3 in the distribution of the computation time $C_{2}=4.2$ of $\tau_{2}$ to its subjobs. Unlike $\mathcal{T}_{5}, \mathcal{T}_{9}$ is not schedulable, i.e. the second job of task $\tau_{2}$ in Fig. 12 misses its deadline upon a simultaneous release of both tasks.

Table 9 Task characteristics of $\mathcal{T}_{9}$

\begin{tabular}{lll}
\hline & $T_{i}=D_{i}$ & $C_{i}$ \\
\hline$\tau_{1}$ & 5 & 2 \\
$\tau_{2}$ & 7 & $2+2.2$ \\
\hline
\end{tabular}

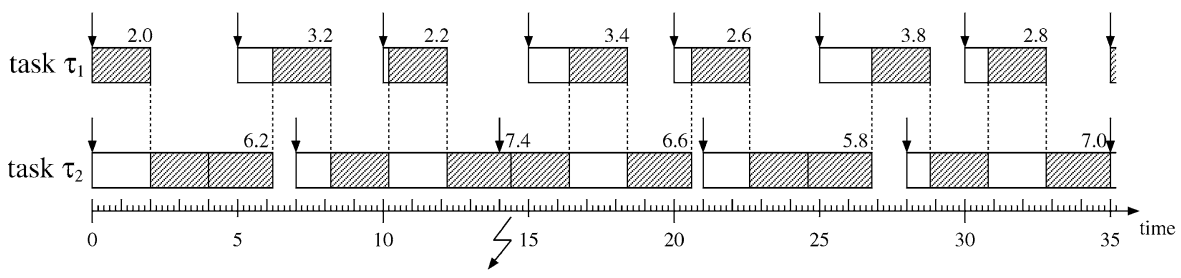

Fig. 12 Timeline for $\mathcal{T}_{9}$ under FPDS with a simultaneous release of all tasks at time zero and a deadline miss of task $\tau_{2}$ at time $t=14$ 


\section{Conclusions}

In this paper, we revisited existing worst-case response time analysis of hard real-time tasks under FPDS, arbitrary phasing and relative deadlines at most equal to periods. We showed by means of a number of examples that existing analysis is pessimistic and/or optimistic, both for FPDS as well as for FPNS, being a special case of FPDS. From these examples, we concluded that the worst-case response time of a task is not necessarily assumed for the first job of a task when released at a critical instant. The reason for this is that the final subjob of a task can defer the execution of higher priority tasks, which can potentially give rise to higher interference for subsequent jobs of that task. This problem can therefore arise for all tasks, except for the highest priority task. We observed that González Harbour et al. (1991) identified the same influence of jobs of a task for relative deadlines at most equal to periods in the context of FPPS of periodic tasks with varying execution priority.

We provided revised worst-case response time analysis, resolving the problems with existing approaches. The analysis is based on known concepts of critical instant and busy period for FPPS, for which we gave slightly modified definitions to accommodate for our scheduling model for FPDS. To prevent confusion with existing definitions of busy period, we used the term active period for our definition in this document. We gave a formal definition of active period, discussed conditions for its termination, and presented a sufficient condition with a formal proof.

We showed that the critical instant, longest active period, and worst-case response time for a task are suprema rather than maxima for all tasks, except for the lowest priority task. Hence, that instant, period, and response time cannot be assumed for any task, except for the lowest priority task. These anomalies for the lowest priority task are caused by the fact that only the lowest priority task cannot be blocked. We expressed worst-case response times under FPDS in terms of worst-case response times and worst-case occupied time under FPPS, and presented an iterative procedure to determine worst-case response times under FPDS.

We briefly compared the notion of level- $i$ active period with similar notions in the literature. We concluded that the notions of $\tau_{i}$-busy period in González Harbour et al. (1991), level-i busy period in George et al. (1996), and level- $\pi_{i}$ busy interval in Liu (2000) are similar to our notion of level- $i$ active period. There are striking differences with the notion of busy period in Lehoczky (1990), however. In particular, the level- $n$ busy period never ends for a utilization factor $U=1$. Moreover, we observed that although Klein et al. (1993) refers to the notion of busy period from Lehoczky (1990) in their description of a method to determine worst-case response times of tasks under FPPS, arbitrary phasing and deadlines larger than periods, their termination condition is actually based on the notion of active period rather than busy period. We also presented uniform, but pessimistic variants of our worst-case response time analysis, and showed that the evolutionary improvement of the analysis for CAN as presented in Davis et al. (2007) corresponds to one of these variants. We illustrated our analysis for an advanced model for FPDS, where tasks are structured as flow graphs of subjobs rather than sequences. Finally, we showed that our analysis for FPDS is sustainable and therefore also applicable for sporadic task systems. 
Acknowledgements We thank Alan Burns and Robert I. Davis from the University of York for discussions, and the IST-004527 funded ARTIST 2 Network of Excellence on Embedded Systems Design for making those discussions possible. We also thank Mike J. Holenderski and the anonymous referees of the Real Time Systems Journal for there comments on earlier versions of this paper.

Open Access This article is distributed under the terms of the Creative Commons Attribution Noncommercial License which permits any noncommercial use, distribution, and reproduction in any medium, provided the original author(s) and source are credited.

\section{Appendix: Auxiliary definitions and lemmas}

This appendix presents auxiliary definitions for greatest common divisor and least common multiple for both positive rational numbers and positive real numbers. Moreover, it presents auxiliary lemmas for a strictly increasing function $f(x)$ and an auxiliary lemma for the ceiling function and the floor function.

Definition 17 The least common multiple for positive rational numbers $\left(1 \mathrm{~cm} \mathbb{Q}^{+}\right)$is defined as

$$
\operatorname{lcm}^{\mathbb{Q}^{+}}\left(r_{1}, \ldots, r_{l}\right)=\min \left\{r \in \mathbb{Q}^{+} \mid r=n_{1} \cdot r_{1}=\cdots=n_{l} \cdot r_{l} \text { with } n_{1}, \ldots, n_{l} \in \mathbb{N}^{+}\right\}
$$

where $l \in \mathbb{N}$ and $l \geq 2$, and $r_{1}, \ldots, r_{l} \in \mathbb{Q}^{+}$.

Definition 18 The greatest common divisor for positive rational numbers $\left(\operatorname{gcd}^{\mathbb{Q}^{+}}\right)$is defined as

$$
\operatorname{gcd}^{\mathbb{Q}^{+}}\left(r_{1}, \ldots, r_{l}\right)=\max \left\{r \in \mathbb{Q}^{+} \mid n_{1} \cdot r=r_{1}, \ldots, n_{l} \cdot r=r_{l} \text { with } n_{1}, \ldots, n_{l} \in \mathbb{N}^{+}\right\},
$$

where $l \in \mathbb{N}$ and $l \geq 2$, and $r_{1}, \ldots, r_{l} \in \mathbb{Q}^{+}$.

Definition 19 The least common multiple for positive real numbers $\left(\mathrm{cm}^{\mathbb{R}^{+}}\right)$is defined as

$$
\operatorname{lcm}^{\mathbb{R}^{+}}\left(r_{1}, \ldots, r_{l}\right)=\min \left\{r \in \mathbb{R}^{+} \mid r=n_{1} \cdot r_{1}=\cdots=n_{l} \cdot r_{l} \text { with } n_{1}, \ldots, n_{l} \in \mathbb{N}^{+}\right\}
$$

where $l \in \mathbb{N}$ and $l \geq 2$, and $r_{1}, \ldots, r_{l} \in \mathbb{R}^{+}$.

Definition 20 The greatest common divisor for positive real numbers $\left(\mathrm{gcd}^{\mathbb{R}^{+}}\right)$is defined as

$$
\operatorname{gcd}^{\mathbb{R}^{+}}\left(r_{1}, \ldots, r_{l}\right)=\max \left\{r \in \mathbb{R}^{+} \mid n_{1} \cdot r=r_{1}, \ldots, n_{l} \cdot r=r_{l} \text { with } n_{1}, \ldots, n_{l} \in \mathbb{N}^{+}\right\},
$$

where $l \in \mathbb{N}$ and $l \geq 2$, and $r_{1}, \ldots, r_{l} \in \mathbb{R}^{+}$.

Unlike $\mathrm{gcd}^{\mathbb{Q}^{+}}$and $\mathrm{lcm} \mathbb{Q}^{+}$, the greatest common divisor for positive real numbers $\mathrm{gcd}^{\mathbb{R}^{+}}$and the least common multiple for positive real numbers $1 \mathrm{~cm}^{\mathbb{R}^{+}}$need not exist. 
Lemma 15 (Bril 2004, Lemma 4.3) Let $f(x)$ be defined and strictly non-decreasing in an interval $[a, b]$ with $f(a)>a$ and $f(b)<b$. Then there exists a value $c \in(a, b)$ such that $f(c)=c$.

Proof See Bril (2004).

Lemma 16 (Bril (2004, Lemma 4.5)) When $\lim _{x \downarrow X} f(x)$ is defined, and $f(x)$ is strictly increasing in an interval $(X, X+\gamma)$ for sufficiently small $\gamma \in \mathbb{R}^{+}$, then the following equation holds:

$$
\lim _{x \downarrow X}\lceil f(x)\rceil=\left\lfloor\lim _{x \downarrow X} f(x)\right\rfloor+1 .
$$

Proof See Bril (2004).

Lemma 17 When $\lim _{x \uparrow X} f(x)$ is defined, and $f(x)$ is strictly increasing in an interval $(X-\gamma, X)$ for a sufficiently small $\gamma \in \mathbb{R}^{+}$, then the following equation holds:

$$
\lim _{x \uparrow X}\lceil f(x)\rceil=\left\lceil\lim _{x \uparrow X} f(x)\right\rceil .
$$

Proof The proof uses the definition of limit:

$$
\lim _{x \uparrow X} f(x)=Y \quad \Leftrightarrow \quad \underset{\varepsilon>0}{\forall} \underset{\delta>0}{\exists} \underset{x \in(X-\delta, X)}{\forall}|f(x)-Y|<\varepsilon .
$$

We first prove the relation

$$
\underset{X-\gamma<x<X}{\forall} f(x)<Y,
$$

and subsequently prove the lemma.

The proof of the relation is based on a contradiction argument. Because $\lim _{x \uparrow X} f(x)$ is defined, we may write $\lim _{x \uparrow X} f(x)=Y$. Assume $f\left(x_{1}\right) \geq Y$ for an $x_{1} \in(X-\gamma, X)$. Choose an $x_{2} \in\left(x_{1}, X\right)$. Because $f(x)$ is strictly increasing in $(X-\gamma, X), f\left(x_{2}\right)>f\left(x_{1}\right) \geq Y$. Now choose $\varepsilon=f\left(x_{2}\right)-Y$, then

$$
\forall_{x \in\left(x_{2}, X\right)} f(x)>f\left(x_{2}\right)>Y
$$

and hence

$$
|f(x)-Y|>\left|f\left(x_{2}\right)-Y\right|=\varepsilon,
$$

which contradicts the fact that $\lim _{x \uparrow X} f(x)=Y$.

For the proof of the lemma, we consider two main cases: $Y \in \mathbb{Z}$ and $Y \notin \mathbb{Z}$. Let $Y \in \mathbb{Z}$. According to the relation proved above, $0<Y-f(x)$ for all $x \in(X-\gamma, X)$. Let $\varepsilon \in(0,1]$. Now there exists a $\delta_{1} \in(0, \gamma)$ such that $0<Y-f(x)<\varepsilon \leq 1$ for all $x \in\left(X-\delta_{1}, X\right)$, hence $Y>f(x)>Y-1$, i.e. $\lceil f(x)\rceil=Y=\lceil Y\rceil$. So,

$$
\lim _{x \uparrow X}\lceil f(x)\rceil=\lim _{x \uparrow X}\lceil Y\rceil=\lceil Y\rceil=\left\lceil\lim _{x \uparrow X} f(x)\right\rceil .
$$


Next, let $Y \notin \mathbb{Z}$. Let $\varepsilon \in(0, Y-\lfloor Y\rfloor]$. Now there exists a $\delta_{2} \in(0, \gamma)$ such that for all $x \in\left(X-\delta_{2}, X\right)$

$$
0<Y-f(x)<\varepsilon \leq Y-\lfloor Y\rfloor,
$$

hence

$$
Y>f(x)>Y-\varepsilon \geq\lfloor Y\rfloor,
$$

i.e.

$$
\lceil f(x)\rceil=\lceil Y\rceil .
$$

For this second main case we therefore also find

$$
\lim _{x \uparrow X}\lceil f(x)\rceil=\lim _{x \uparrow X}\lceil Y\rceil=\lceil Y\rceil=\left\lceil\lim _{x \uparrow X} f(x)\right\rceil,
$$

which proves the lemma.

The proofs of the following two lemmas are similar to the proofs of the previous two lemmas.

Lemma 18 When $\lim _{x \uparrow X} f(x)$ is defined, and $f(x)$ is strictly increasing in an interval $(X-\gamma, X)$ for a sufficiently small $\gamma \in \mathbb{R}^{+}$, then the following equation holds:

$$
\lim _{x \uparrow X}\lfloor f(x)\rfloor=\left\lceil\lim _{x \uparrow X} f(x)\right\rceil-1 .
$$

Lemma 19 When $\lim _{x \downarrow X} f(x)$ is defined, and $f(x)$ is strictly increasing in an interval $(X, X+\gamma)$ for sufficiently small $\gamma \in \mathbb{R}^{+}$, then the following equation holds:

$$
\lim _{x \downarrow X}\lfloor f(x)\rfloor=\left\lfloor\lim _{x \downarrow X} f(x)\right\rfloor .
$$

Lemma 20 For $n \in \mathbb{Z}, m \in \mathbb{Z} \backslash\{0\}$, and $\varepsilon \in \mathbb{R}$, the following relation holds:

$$
0<\varepsilon<1 \Rightarrow\left\lceil\frac{n+\varepsilon}{m}\right\rceil=\left\lfloor\frac{n}{m}\right\rfloor+1 .
$$

Proof The proof is based on properties of ceiling and floor functions. Let

$$
\left\lceil\frac{n+\varepsilon}{m}\right\rceil=l
$$

with $l \in \mathbb{Z}$, hence

$$
m(l-1)<n+\varepsilon \leq m l .
$$

For $0<\varepsilon<1$, we get $n+\varepsilon \notin \mathbb{Z}$ and $n<n+\varepsilon<n+1$. Moreover, because $m(l-1), m l \in \mathbb{Z}$, we derive

$$
0<\varepsilon<1 \Rightarrow(m(l-1)<n+\varepsilon \leq m l \Leftrightarrow m(l-1) \leq n<m l) .
$$


With $m \neq 0$, we derive from $m(l-1) \leq n<m l$ that

$$
\left\lfloor\frac{n}{m}\right\rfloor=l-1 \text {, }
$$

which proves the lemma.

\section{References}

Audsley NC, Burns A, Richardson MF, Wellings AJ (1991) Hard real-time scheduling: The deadline monotonic approach. In: Proc of the 8th IEEE workshop on real-time operating systems and software (RTOSS), May 1991, pp 133-137

Baeten JCM, Middelburg CA (2002) Process algebra with timing. Springer, Berlin

Baruah S (2005) The limited-preemption uniprocessor scheduling of sporadic systems. In: Proc of the 17th Euromicro conference on real-time systems (ECRTS), July 2005, pp 137-144

Baruah S, Burns A (2006) Sustainable schedulability analysis. In: Proc of the 27th IEEE real-time systems symposium (RTSS), December 2006, pp 159-168

Baruah SK, Mok AK, Rosier LE (1990a) Preemptively scheduling hard-real-time sporadic tasks on one processor. In: Proc of the 11th IEEE real-time systems symposium (RTSS), December 1990, pp 182190

Baruah SK, Rosier LE, Howell RR (1990b) Algorithms and complexity concerning the preemptive scheduling of periodic, real-time tasks on one processor. Real-Time Syst 2(4):301-324

Bril RJ (2004) Real-time scheduling for media processing using conditionally guaranteed budgets. $\mathrm{PhD}$ thesis, Technische Universiteit Eindhoven (TU/e), The Netherlands. http://alexandria.tue.nl/extra2/ 200412419.pdf

Bril RJ (2006) Existing worst-case response time analysis of real-time tasks under fixed-priority scheduling with deferred preemption is too optimistic. Technical report CS 06-05, Department of Mathematics and Computer Science, Technische Universiteit Eindhoven (TU/e), The Netherlands, February 2006

Bril RJ, Verhaegh WFJ, Lukkien JJ (2004) Exact worst-case response times of real-time tasks under fixedpriority scheduling with deferred preemption. In: Proc of the work-in-progress (WiP) session of the 16th Euromicro conference on real-time systems (ECRTS), Technical report from the University of Nebraska-Lincoln, Department of Computer Science and Engineering (TR-UNL-CSE-2004-0010), June 2004, pp 57-60

Bril RJ, Lukkien JJ, Davis RI, Burns A (2006) Message response time analysis for ideal controller area network (CAN) refuted. In: Proc of the 5th international workshop on real time networks (RTN), July 2006, pp 13-18

Bril RJ, Lukkien JJ, Verhaegh WFJ (2007) Worst-case response time analysis of real-time tasks under fixed-priority scheduling with deferred preemption revisited. In: Proc of the 19th Euromicro conference on real-time systems (ECRTS), July 2007, pp 269-279

Burns A (1994) Preemptive priority based scheduling: An appropriate engineering approach. In: Son S (ed) Advances in real-time systems. Prentice-Hall, Englewood Cliffs, pp 225-248

Burns A (2001) Defining new non-preemptive dispatching and locking policies for Ada. In: Proc of the 6th Ada-Europe international conference, May 2001. Lecture notes in computer science, vol 2043. Springer, Berlin, pp 328-336

Burns A, Wellings AJ (1997) Restricted tasking models. In: Proc of the 8th international real-time Ada workshop, pp 27-32

Burns A, Nicholson M, Tindell K, Zhang N (1993) Allocating and scheduling hard real-time tasks on a point-to-point distributed system. In: Proc of the 1st workshop on parallel and distributed real-time systems, April 1993, pp 11-20

Buttazzo GC (2005) Hard real-time computing systems-predictable scheduling algorithms and applications, 2nd edn. Springer, Berlin

Davis RI, Burns A, Bril RJ, Lukkien JJ (2007) Controller area network (CAN) schedulability analysis: refuted, revisited and revised. Real-Time Syst 35(3):239-272

George L, Rivierre N, Spuri M (1996) Preemptive and non-preemptive real-time uni-processor scheduling. Technical report 2966, Institut National de Recherche en Informatique et en Automatique (INRIA), France, September 1996 
González Harbour M, Klein MH, Lehoczky JP (1991) Fixed-priority scheduling with varying execution priority. In: Proc of the 12th IEEE real-time systems symposium (RTSS), December 1991, pp 116128

Gopalakrishnan R, Parulkar GM (1996) Bringing real-time scheduling theory and practice closer for multimedia computing. In: Proc of the ACM Sigmetrics conference on measurement \& modeling of computer systems, May 1996, pp 1-12

Hermant J-F, Leboucher L, Rivierre N (1996) Real-time fixed and dynamic priority driven scheduling algorithms: theory and practice. Technical report 3081, Institut National de Recherche en Informatique et en Automatique (INRIA), France, December 1996

Hooman J (1991) Specification and compositional verification of real-time systems. PhD thesis, Technische Universiteit Eindhoven (TU/e), The Netherlands

Joseph M, Pandya P (1986) Finding response times in a real-time system. Comput J 29(5):390-395

Klein MH, Ralya T, Pollak B, Obenza R, González Harbour M (1993) A practitioner's handbook for realtime analysis: guide to rate monotonic analysis for real-time systems. Kluwer Academic, Dordrecht

Koymans R (1990) Specifying real-time properties with metric temporal logic. Real-Time Syst 2(4):255299

Lee S, Lee C-G, Lee M, Min SL, Kim C-S (1998) Limited preemptible scheduling to embrace cache memory in real-time systems. In: Proc of the ACM Sigplan workshop on languages, compilers and tools for embedded systems (LCTES), June 1998. Lecture notes in computer science, vol 1474. Springer, Berlin, pp 51-64

Lehoczky JP (1990) Fixed priority scheduling of periodic task sets with arbitrary deadlines. In: Proc of the 11th IEEE real-time systems symposium (RTSS), December 1990, pp 201-209

Liu JWS (2000) Real-time systems. Prentice-Hall, Englewood Cliffs

Liu CL, Layland JW (1973) Scheduling algorithms for multiprogramming in a real-time environment. J ACM 20(1):46-61

Mok AK-L (1983) Fundamental design problems of distributed systems for the hard-real-time environment. PhD thesis, Massachusetts Institute of Technology. http://www.lcs.mit.edu/publications/pubs/ pdf/MIT-LCS-TR-297.pdf

Mok AK, Poon W-C (2005) Non-preemptive robustness under reduced system load. In: Proc of the 26th IEEE real-time systems symposium (RTSS), December 2005, pp 200-209

Regehr J (2002) Scheduling tasks with mixed preemption relations for robustness to timing faults. In: Proc of the 23rd IEEE real-time systems symposium (RTSS), December 2002, pp 315-326

Sha L, Rajkumar R, Lehoczky JP (1990) Priority inheritance protocols: an approach to real-time synchronisation. IEEE Trans Comput 39(9):1175-1185

Simonson J, Patel JH (1995) Use of preferred preemption points in cache-based real-time systems. In: Proc of the IEEE international computer performance and dependability symposium (IPDS), April 1995, pp 316-325

Spuri M (1996) Analysis of deadline scheduled real-time systems. Technical report 2772, Institut National de Recherche en Informatique et en Automatique (INRIA), France, January 1996

Tindell K, Burns A (1994) Guaranteeing message latencies on controller area network (CAN). In: Proc of the 1st international CAN conference, September 1994, pp 1-11

Tindell K, Hansson H, Wellings AJ (1994) Analysing real-time communications: Controller area network (CAN). In: Proc of the 15th IEEE real-time systems symposium (RTSS), December 1994, pp 259263

Tindell K, Burns A, Wellings AJ (1995) Calculating controller area network (CAN) message response times. Control Eng Pract 3(8):1163-1169

Wang Y, Saksena M (1999) Scheduling fixed-priority tasks with preemption threshold. In: Proc of the 6 th international conference on real-time computing systems and applications (RTCSA), December 1999, pp 328-335

Weisstein EW (2003) CRC Concise Encyclopedia of Mathematics. Chapman \& Hall/CRC, London 

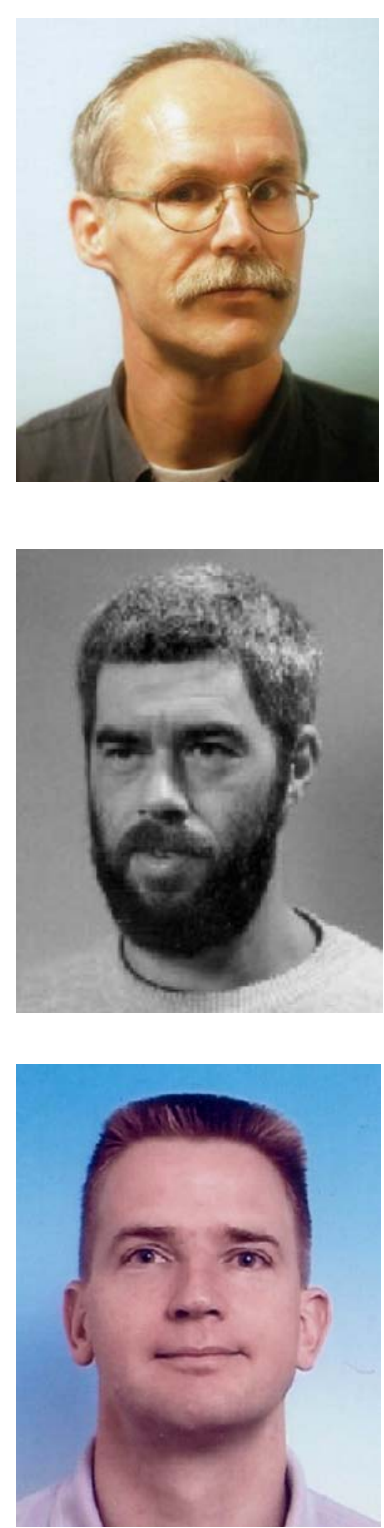

Reinder J. Bril received a B.Sc. and an M.Sc. (both with honors) from the University of Twente, and a Ph.D. from the Technische Universiteit Eindhoven, the Netherlands. He started his professional career in January 1984 at the Delft University of Technology. From May 1985 till August 2004, he has been with Philips, and worked in both Philips Research as well as Philips' Business Units. He worked on various topics, including fault tolerance, formal specifications, software architecture analysis, and dynamic resource management, and in different application domains, e.g. high-volume electronics consumer products and (low volume) professional systems. In September 2004, he made a transfer back to the academic world, i.e. to the System Architecture and Networking (SAN) group of the Mathematics and Computer Science department of the Technische Universiteit Eindhoven. His main research interests are currently in the area of reservation-based resource management for networked embedded systems with real-time constraints.

Johan J. Lukkien is head of the System Architecture and Networking Research group at Eindhoven University of Technology since 2002. He received M.Sc. and Ph.D. from Groningen University in the Netherlands. In 1991 he joined Eindhoven University after a two years leave at the California Institute of Technology. His research interests include the design and performance analysis of parallel and distributed systems. Until 2000 he was involved in large-scale simulations in physics and chemistry. Since 2000, his research focus has shifted to the application domain of networked resource-constrained embedded systems. Contributions of the SAN group are in the area of component-based middleware for resource-constrained devices, distributed coordination, Quality of Service in networked systems and schedulability analysis in real-time systems.

Wim F.J. Verhaegh received the mathematical engineering degree with honors in 1990 from the Technische Universiteit Eindhoven, the Netherlands. Since then, he is with the Philips Research Laboratories in Eindhoven, the Netherlands. From 1990 until 1998, he has been a member of the department Digital VLSI, where he has been working on high-level synthesis of DSP systems for video applications, with the emphasis on scheduling problems and techniques. Based on this work, he received a Ph.D. degree in 1995 from the Technische Universiteit Eindhoven. Since 1998, he is working on various optimization aspects of multimedia systems, networks, and applications. On the one hand, this concerns application-level resource management and scheduling, for optimization of quality of service of multimedia systems. On the other hand, this concerns adaptive algorithms and machine learning algorithms for user interaction issues, such as content filtering and automatic playlist generation. 